\title{
THE THEORY OF MULTIPLICATIVE ARITHMETIC FUNCTIONS*
}

\author{
BY \\ R. VAIDYANATHASWAMY \\ INTRODUCTION
}

1. The work the results of which are embodied in this paper was carried out during the period from August, 1927, to October, 1928, when the paper was sent for publication in these Transactions. In October, 1927, I contributed a note $O n$ the inversion of multiplicative arithmetic functions to the Journal of the Indian Mathematical Society, pointing out the fact (which I then believed to be new) that every multiplicative function of a single argument possesses an inverse, which is also a multiplicative function. In this note, certain of the ideas of the earlier part of this paper are presented in an undeveloped form; in particular, there occur the term "linear function" and the notion of "rational integral function." This note called forth, a year later, a paper entitled An outline of a theory of arithmetic functions, by E. T. Bell (Journal of the Indian Mathematical Society, October, 1928), wherein he pointed out that he had established the existence of the inverse function, for a wider class of functions than the multiplicative, and gave a general survey, with full references, of his own work on numerical functions. Such of the literature indicated by this extremely useful paper of Dr. Bell as was then available to me, showed that the particular types of problems, for which I was interested in finding a solution, had not been considered previously. When, two or three months after the paper had left my hands, his memoir An arithmetical theory of certain numerical functions (University of Washington Publications in Mathematical and Physical Sciences, vol. 1, No. 1, 1915) became available for reference, it became apparent that some of his ideas were in close relation with the part of this paper (namely, the first three sections) which deals specifically with functions of a single argument. (A precise account of the relation is given in $\$ 4$ of this Introduction.)

Some of my main results were communicated early in 1928 to the International Congress of Mathematics, Bologna, but the abstract published in the Acts of the Congress is imperfect, and does not cover the whole ground of this paper.

* Presented to the Society, April 3, 1931; received by the editors in November, 1928, and revised, with new introduction, in December, 1930. 
In revising the paper, some explanatory passages and further references have been inserted, but little new matter has been added to the text.

2. The arithmetical functions $f(N)$ which have the property that $f(M N)$ $=f(M) f(N)$ when $M, N$ are mutually prime, are well known, and are of paramount importance in arithmetic; the functions of $r$ arguments which possess the corresponding property

$$
f\left(M_{1} N_{1}, M_{2} N_{2}, \cdots, M_{r} N_{r}\right)=f\left(M_{1}, M_{2}, \cdots, M_{r}\right) f\left(N_{1}, N_{2}, \cdots, N_{r}\right),
$$

when the two products $M_{1} M_{2} \cdots M_{r}, N_{1} N_{2} \cdots N_{r}$ are relatively prime, are less widely known.* The functions with this property have received several names, and are here called "multiplicative." $\dagger$ Though some of the processes and results of this paper could be stated for a wider class of arithmetic functions, it has been thought desirable to confine it strictly to the functions with this property, so that "function" used here without any qualification, means always "multiplicative arithmetic function."

Though multiplicative functions of a single argument are widely known and used, they have not been studied, as such, by any writer before Bell; this indeed may be inferred from the fact that there is no recognized name for the fundamental process relating to them, here called "composition." Bell has termed this process "ideal multiplication," referring, for distinction, to the ordinary product of two functions, as their "algebraic" or "absolute" product. $\ddagger$ The process of convolution of arguments, which is the logical basis of composition, does not appear to be known at all, though $\mathrm{I}$ have seen it used in a solitary instance, for a function of two arguments by Ramanujan. $\S$

In Section I fundamental concepts are defined, and certain elementary functions are introduced; the method of generating series is explained, and the independence of the elements of a multiplicative function is affirmed.

Section II studies the five fundamental processes of the calculus, multiplication, convolution, composition, inversion and compounding, the last of these being new. Composition is really a particular case, though a most

* I am not aware of any writer other than Bell who has treated these functions: see E. T. Bell, $A$ ray of numerical functions of $r$ arguments, Bulletin of the American Mathematical Society, vol. 32 (1926), p. 341.

$\dagger$ Bell uses "factorable," and refers (Journal of the Indian Mathematical Society, October, 1928) to the German and French equivalents "zerlegbar" and "régulière," which I have not seen. I have adopted "multiplicative" from Pólya and Szegö, Aufgaben und Lehrsätze aus der Analysis, vol. II, p. 126 , where it is used in this sense.

$\ddagger$ An arithmetical theory of numerical functions (loc. cit.) and An outline etc. (loc. cit.).

$\S$ Collected Papers of S:inivasa Ramanujan, Cambridge, 1927, p. 180.

|| Bell was led, in 1915, in a purely symbolic manner, to the compounding operation, by working with "generators," without being aware of its arithmetical significance. He called it "ideal addition." The names "ideal multiplication" and "ideal addition" appear to have been chosen under the mistaken impression that the latter distributes the former; see Introduction (4). 
important one, of convolution, which is logically prior to it; this is not however usually recognized, owing to the fact that in order to explain composition in terms of convolution (as is done here), one must work from the start with functions of several arguments. The theory of inversion in its widest form is due to Bell.*

Section III studies rational functions of a single argument, proves the result that multiplication and compounding are rational processes, and gives various applications.

Sections IV and V, though both of independent interest as treating of important special types of multiplicative functions of several arguments, are intended to be preparatory to Section VI, which investigates a general form of identical relation, which is satisfied by every multiplicative function. The special functions are also determined, for which this relation reduces to a "Busche-Ramanujan identity," namely, an identity which, for functions $f$ of a single argument, is of the form

$$
f(M N)=\sum f\left(\frac{M}{\delta}\right) f\left(\frac{N}{\delta}\right) F(\delta)
$$

summed for all common divisors $\delta$ of $M, N$. The function $\sigma_{a}(N)$, representing the sum of the ath powers of the divisors of $N$, is the known instance of a function admitting an identity of this type. On the other hand, the $\phi$-function of Euler also satisfies an identity of the same form, namely

$$
\phi(M N)=\sum \delta \phi\left(\frac{M}{\delta}\right) \phi\left(\frac{N}{\delta}\right)
$$

summed for common divisors $\delta$ of $M, N$, provided the values of $M, N$, are so restricted as not to contain any common prime factor to the same power. $\dagger$ All functions of a single argument which admit a "restricted Busche-Ramanujan identity" of this kind are also determined.

The seventh and last section finds the general form of a multiplicative function, which can constitute the general element of a determinant capable of evaluation by the same method as Smith's determinant. Two new forms of such determinants are added here to those already known, namely, the determinants whose general element $a_{m n}$ is equal to (1) von Sterneck's func-

* On a certain inversion in the theory of numbers, Tohoku Mathematical Journal, vol. 17 (1920), p. 221; Extension of Dirichlet multiplication and Dedekind inversion, Bulletin of the American Mathematical Society, vol. 28 (1922), p. 111.

$\dagger$ This property of the $\phi$-function was discovered by Mr. S. Sivasankaranarayana Pillai, while a research scholar of the Madras University; it suggested to the author the concept of "restricted identity." 
tion $f(m, n),(2)$ any "integral quadratic function" of the product $m n$, e.g., the sum of the divisors of $m n$ or the number of representations of $m n$ as a sum of two squares.

3. It may perhaps help the reader to follow the paper with greater understanding, if I explain the exact manner in which it arose. The paper is mainly an attempt to understand the well known identity

$$
\sigma_{a}(m n)=\sum \sigma_{a}\left(\frac{m}{\delta}\right) \sigma_{a}\left(\frac{n}{\delta}\right) \delta^{a} \mu(\delta)
$$

summed for common divisors $\delta$ of $m$, $n$, and to answer the converse problem suggested by it, of finding the most general function admitting an identity of this form.

It is also, to a lesser extent, a study of two other particular results:

$$
\sum_{n=1}^{\infty} \frac{\sigma_{a}(n) \sigma_{b}(n)}{n^{s}}=\frac{\zeta(s) \zeta(s-a) \zeta(s-b) \zeta(s-a-b)}{\zeta(2 s-a-b)} \text { (Ramanujan). }
$$

(C) The determinant $\left|a_{r s}\right|(r, s=1,2, \cdots, N)$, where $a_{r s}$ is the least common multiple of $r$ and $s$, has the value $\prod_{j=1}^{N} \phi(j) \prod(j)$, where $\phi$ is Euler's function and $\Pi(j)$ is the product of the negatives of the prime factors of $j$ (Cesàro).

At a fairly early stage in the work, I succeeded in proving that the most general multiplicative function $f(N)$ admitting an identity of the form (A) must be an "integral quadratic function." Thus, it is not possible to answer the converse question in (A), without the concepts of "linear" and "rational integral" functions. At the same time I had grasped the fact that the identity (A) could not be properly understood, unless $\sigma_{a}(M N)$ was treated explicitly as a multiplicative function of the two arguments $M, N$, and its right side, as the composite of two functions, each of two arguments; from this point of view $F(\delta)=\delta^{a} \mu(\delta)$ can only be described as "the function of one argument equivalent to a 'principal' function of two arguments." The more difficult question of the form of the corresponding identity for the general function $f(N)$ was solved much later, giving the concepts "cardinal function," and "conjugate function," so that it became possible to describe the specialization which occurred when $f$ became integral-quadratic, as the specialization of a cardinal function into a principal function. Also, I had previously been led to the compounding process, in working at problems involving the l.c.m. and g.c.d. of divisors of a number, but had felt doubtful whether it should be taken seriously; the occurrence of the "conjugate function" in this context convinced me that the compounding process should be given as fundamental a place as composition. In the last stage the whole theory was generalized and stated 
for functions of $r$ arguments, thereby necessitating the concepts "cardinal and principal functions of a matrix-set of arguments." Thus in all (A) has been responsible for (1) acceptance and systematic treatment of multiplicative functions of more than one argument; (2) the concept of rational function; (3) the theory of cardinal and principal functions; (4) the systematic study of the compounding operation. The secondary line of thought indicated by the restricted Busche-Ramanujan identity joins on with (A); it had its genesis in the attempt to understand the property of the $\phi$-function (already mentioned), which Mr. S. S. Pillai communicated to me in answer to the problem which I proposed to him for solution, namely, either to explain in a satisfactory manner why Euler's function does not satisfy an identity of the type (A), or to find some altered form of the identity which it does satisfy.

After the concept "rational function" had been fully formed, the result (B) was recognized as giving "the expression in rational form, of the product of the two integral quadratic functions $\sigma_{a}$ and $\sigma_{b}$." This suggested the result that the product of two rational functions is rational and raised the question of expressing it in rational form. In regard to this question, it was easily proved that the "integral component" of the product-function was capable of immediate derivation from the integral components of the factors; but no result, more general than the theorem of III $\$ 4$, was obtained in spite of prolonged effort, for the specification of the inverse component. The immediate extension of (B) to the product of two general integral quadratic functions was proved both from the general theory, and by arithmetical methods utilizing the compounding operation (Example 9 and III $\$ 6(\mathrm{~b})$ ). The theory of simplex functions (II $\$ 5(d)$ ) has also been suggested by (B).

The result $(\mathrm{C})$ has necessitated the close study of functions of the g.c.d. and l.c.m., the former joining on with the theory of principal functions, while the latter gives rise to the new concept of "semiprincipal function." In Smith's original statement of his determinant-theorem, any multiplicative function of the g.c.d. of $m, n$ can serve as the general element $a_{m n}$ of his determinant, ${ }^{*}$ whereas in Cesàro's extension, $\dagger$ it is only the linear function of the l.c.m. of $m, n$ which can so serve. This difference in character between the l.c.m. and the g.c.d. is explained by the fact that the "semiprincipal" function assumes a special form suitable for the production of a "Smith function," only when its equivalent function is an "enumerative totient" (cf. Example 2, VII).

4. The relation between the first three sections and Bell's memoir $A \boldsymbol{n}$ arithmetical theory of certain numerical functions. This memoir studies com-

* Dickson, History of the Theory of Numbers, vol. 1, pp. 122, 123.

† Dickson, ibid., p. 128. 
position under the name "ideal multiplication." Its main object appears to be the selection of a suitable subclass of multiplicative functions $f(N)$, admitting a theorem of unique (compositional) factorization into primes, and the associated arithmetical theory, and the illustration therefrom of general principles relating to arithmetical structure. The "generator" of a multiplicative function $f(N)$ is a function $f(x, z)$ of two arguments, such that, for every prime $p$,

$$
f(p, z)=f(p) z+f\left(p^{2}\right) z^{2}+\cdots .
$$

The "generating function" $F(x, z)$ of $f(N)$ is defined by

$$
F(x, z)=1+f(x, z) .
$$

The functions $f(N)$, whose generating functions $F(x, z)$ are finite polynomials in both $x$ and $z$, are called "primitive" functions, and also "positive" functions; if in addition $F(x, z)$ is an irreducible polynomial in $x, z, f(N)$ is called a "prime primitive." The inverse of a positive function is called a "negative" function, and the composite of a positive and a negative function is a "mixed function." Thus the mixed functions are those whose generating functions $F(x, z)$ are rational functions of $(x, z)$, and it is shown that they admit of a unique factorization theorem, corresponding to the factorization of the numerator and denominator of $F(x, z)$ into their irreducible polynomial factors. The mixed functions are special types of the rational functions of this paper. ${ }^{*}$ It is clear that, if there had been no insistence on the variable $x$ in the generating function, the theory reached would have been identical with that of this paper; but in that case, the purpose of the memoir would not have been fulfilled, since rational functions admit of (compositional) factorization, in an unenumerably infinite number of ways (cf. I $\$ 3$ and remarks on Theorem II). The whole difference of outlook turns upon the difference in procedure between defining $f(N)$ by means of a single function $F(x, z)$, "the generating function," and defining it by means of an infinity of generating series $F(p, z)$, where $p$ stands for each prime in turn. In actual application there may not appear to be much difference between these, but theoretically there is this profound distinction, that the latter definition affirms the independence of the elements of the general function $f$, while the former denies it.

* The inversion in the nomenclature, indicative of the difference in view point, may be noted; namely, the "negative" and the "positive" functions of Bell are, respectively, rational integral functions and their inverses. From the view point of arithmetical structure, the functions $f(N)$ whose $F(x, z)$ is a finite polynomial are fundamental, and are therefore called positive functions by Bell. On the other hand, from the view point of the multiplicative property, the functions $f(N)$ which possess it unconditionally are the fundamental ones, and should be termed "linear integral," even though their generating series to any base is the expansion of a fraction of the form $1 /(1-a r)$. 
Thus, the concept of rational function is present in the memoir, but does not reach full clarity, as it is not freed from the admixture of elements extraneous to the nature of the multiplicative function.

The memoir also defines the compounding operation, under the name "ideal addition," in a purely symbolical manner, from the addition of generators, the author indeed appearing to believe that it does not possess any simple arithmetical significance.* The ideal difference is also defined in the same manner, without the concept of the conjugate function. It is stated without proof on page 32, 5.34, and repeated on page 35, 6.26, that ideal addition distributes ideal multiplication; that this is erroneous is shown by Theorem XIV of this paper, which proves that compounding is not distributive but only quasidistributive, in respect to composition. $\dagger$

\section{Section I. Preliminary}

1. Definition. An arithmetic function $f\left(M_{1}, M_{2}, \cdots, M_{r}\right)$ is one which is defined for all (non-zero) positive values of its arguments. The arithmetic function $f\left(M_{1}, M_{2}, \cdots, M_{r}\right)$ is multiplicative, if

$$
f\left(M_{1} N_{1}, M_{2} N_{2}, \cdots, M_{r} N_{r}\right)=f\left(M_{1}, M_{2}, \cdots, M_{r}\right) f\left(N_{1}, N_{2}, \cdots, N_{r}\right),
$$

whenever the products $M_{1} M_{2} \cdots M_{r}, N_{1} N_{2} \cdots N_{r}$ are relatively prime. With the convention that unity is both prime to and a factor of every num. ber, we see that $f\left(M_{1}, 1, \cdots, 1\right)=1$; or

THEOREM I. Every multiplicative function takes the value unity, for simultaneous unit values of the arguments.

2. The elements of a multiplicative function. Let the arguments $M_{1}, M_{2}, \cdots, M_{r}$ of $f\left(M_{1}, M_{2}, \cdots, M_{r}\right)$ be resolved into their prime factors, so that

$$
M_{i}=p_{1}^{a_{1 i}} p_{2}^{a_{2 i}} p_{3}^{a_{3 i}}\left(i=1,2, \cdots, r ; p_{1}<p_{2}<p_{3}<\cdots\right),
$$

the $a_{i k}$ being zero, or positive integers. Then it follows from the multiplicative property, that

$$
f\left(M_{1}, M_{2}, \cdots, M_{r}\right)=\prod_{\lambda} f\left(p_{\lambda}^{a_{\lambda_{1}}}, p_{\lambda}^{a_{\lambda}}, \cdots, p_{\lambda}^{a_{\lambda r}}\right) \quad(\lambda=1,2, \cdots) .
$$

We shall mean by the element of the multiplicative function $f$ to the base $p_{\lambda}$ the aggregate of values $f\left(p_{\lambda}{ }^{t_{1}}, p_{\lambda}{ }^{t_{2}}, \cdots, p_{\lambda}{ }^{t_{r}}\right)$, for all zero and positive integral

* "It is clear that in no quantitative sense is an ideal sum or difference a sum or difference; the ideal sum expresses a relation between functions, which is only remotely connected with their arguments"; p. 32, 5.33 .

$\dagger$ Introduction dated November 2, 1930. 
values of $t_{1}, t_{2}, \cdots, t_{r}$. Thus the values of $f$ for arbitrary arguments can be found by multiplication, if the elements of $f$ to every prime base are known. In other words, the elements of a multiplicative function completely determine the function.

3. The independence of the elements. The definition of the multiplicative function $f$ implies no necessary relation between its elements. In fact, not only can the system of values which constitute an element be chosen in an arbitrary manner, but also the $\infty^{1}$ elements can be chosen in entire independence of one another. It therefore appears that the general multiplicative function falls apart into a series of elements, which while unrelated to one another, generate the function by multiplication.

This independence of the elements is the most characteristic property of the multiplicative function, and our method of generating series is based directly upon it. It introduces, however, an element of indefiniteness into the multiplicative function, allowing it, as it were, an infinity of degrees of freedom. To illustrate, let $f_{1}, f_{2}$ be two function-types, both possessing a multiplicative property $P$ (i.e., a property which is implied by a property of the elements of the function only). Then each of the unenumerably infinite number of functions $f$, whose elements with respect to certain prime bases are the corresponding elements of $f_{1}$, and with respect to the remaining bases, the elements of $f_{2}$, also possess the property $P$. Hence, when $f_{1}, f_{2}$ are solutions of the problem of determining the functions with the property $P$, each of the unenumerably infinite number of crosses $f$ between $f_{1}, f_{2}$ is also a solution. Several examples of this will occur in this paper.

4. Generating series. With each element to a base $p_{\lambda}$ of the multiplicative function $f\left(M_{1}, M_{2}, \cdots, M_{r}\right)$ we associate the power series

$$
f\left(p_{\lambda}\right)\left(x_{1}, x_{2}, \cdots, x_{r}\right)=\sum f\left(p_{\lambda}^{m_{1}}, p_{\lambda}^{m_{2}}, \cdots, p_{\lambda}^{m_{r}}\right) x_{1}^{m_{1}} x_{2}^{m_{2}} \cdots x_{r}^{m_{r}} .
$$

We call this power series the generating series of $f$ to the base $p_{\lambda}$. From Theorem I, it follows that the constant term in every generating series is unity.

If the generating series is the formal expansion of a function $f_{\left(p_{\lambda}\right)}\left(x_{1}, x_{2}, \cdots, x_{r}\right)$ we call this latter the generating function of $f$ to the base $p_{\lambda}$.

We shall generally use the generating series as the representative of the corresponding element of the function. The variables $x_{1}, x_{2}, \cdots, x_{r}$ are not to be regarded as quantities, but purely as algebraic symbols, which are in formal correspondence with the arguments, and exhibit the element in an ordered shape; thus no questions of convergence of the generating series can arise. 
5. Linear functions. The multiplicative function $f\left(M_{1}, \cdots, M_{r}\right)$ will be called a linear function, if the equation

$$
f\left(M_{1} N_{1}, M_{2} N_{2}, \cdots, M_{r} N_{r}\right)=f\left(M_{1}, M_{2}, \cdots, M_{r}\right) f\left(N_{1}, N_{2}, \cdots, N_{r}\right)
$$

holds not merely when $M_{1} M_{2} \cdots M_{r}$ is prime to $N_{1} N_{2} \cdots N_{r}$, but for all values of $M_{i}, N_{i}(i=1,2, \cdots, r)$.

It is clear that a linear function of $r$ arguments can be expressed as the product of $r$ linear functions, each of one of the arguments. Thus, from (1), we have

$f\left(M_{1}, M_{2}, \cdots, M_{r}\right)=f\left(M_{1}, 1, \cdots, 1\right) f\left(1, M_{2}, 1, \cdots, 1\right) \cdots f\left(1,1, \cdots, 1, M_{r}\right) ;$ and $f\left(M_{1}, 1, \cdots, 1\right)$ is a linear function of the argument $M_{1}$.

The generating function to the base $p$ of a linear function $f(M)$ is

$$
\begin{aligned}
f_{(p)}(x) & =1+f(p) x+f\left(p^{2}\right) x^{2}+\cdots \\
& =1+f(p) x+\{f(p)\}^{2} x^{2}+\cdots \\
& =\frac{1}{1-a x}, \text { if } f(p)=a .
\end{aligned}
$$

Similarly the generating function to the base $p$ of a linear function of $r$ arguments is of the form $\left(1-a_{1} x_{1}\right)^{-1}\left(1-a_{2} x_{2}\right)^{-1} \ldots\left(1-a_{r} x_{r}\right)^{-1}$.

6. The elementary functions. These are of fundamental importance, and may be divided into four groups:

(1) the $E$-functions,

(2) the $\lambda$-functions,

(3) the $I$-functions,

(4) the power units $\pi_{k}, \epsilon_{k}$.

We shall first define these functions for a single argument.

$E_{k}(M)=k^{\nu} ; \nu=$ the number of different prime factors of $M$. For $k$ a positive integer, $E_{k}(M)$ may also be defined as the number of decompositions of $M$ into $k$ factors every two of which are mutually prime.

$\lambda_{k}(M)$ is defined to be the linear function, which takes the value $k$ when $M$ is a prime; hence $\lambda_{k}(M)=k^{\nu} ; \nu=$ total number of prime factors of $M$, multiple prime factors being counted as often as their multiplicity.

The general linear function of $M$ is obviously a cross between an infinity of functions $\lambda_{k}$.

$I_{k}(M)$ is the linear function $M^{k}$.

The power units $\pi_{k}, \epsilon_{k}$ are defined (for positive integral values of $k$ ) as follows: 


$$
\begin{aligned}
& \pi_{k}(M)=\left\{\begin{array}{l}
0 \text { if } M \text { is divisible by a } k \text { th power, } \\
1 \text { otherwise }
\end{array}\right. \\
& \epsilon_{k}(M)=\left\{\begin{array}{l}
1 \text { if } M \text { is a } k \text { th power } \\
0, \text { otherwise }
\end{array}\right.
\end{aligned}
$$

The corresponding elementary functions of $r$ arguments are defined by

$$
\begin{aligned}
E_{k}\left(M_{1}, M_{2}, \cdots, M_{r}\right) & =E_{k}\left(M_{1} M_{2} \cdots M_{r}\right) ; \\
& =k
\end{aligned}
$$

if $M_{1}, M_{2}, \cdots, M_{r}$ are all powers of a prime $p$;

$$
\begin{aligned}
& \lambda_{k}\left(M_{1}, M_{2}, \cdots, M_{r}\right)=\lambda_{k}\left(M_{1} M_{2} \cdots M_{r}\right)=\lambda_{k}\left(M_{1}\right) \lambda_{k}\left(M_{2}\right) \cdots \lambda_{k}\left(M_{r}\right) ; \\
& I_{k}\left(M_{1}, M_{2}, \cdots, M_{r}\right)=I_{k}\left(M_{1} M_{2} \cdots M_{r}\right)=I_{k}\left(M_{1}\right) I_{k}\left(M_{2}\right) \cdots I_{k}\left(M_{r}\right) ; \\
& \pi_{k}\left(M_{1}, M_{2}, \cdots, M_{r}\right)=\pi_{k}\left(M_{1}\right) \cdots \pi_{k}\left(M_{r}\right) ; \\
& \epsilon_{k}\left(M_{1}, \cdots, M_{r}\right)=\epsilon_{k}\left(M_{1}\right) \epsilon_{k}\left(M_{2}\right) \cdots \epsilon_{k}\left(M_{r}\right) .
\end{aligned}
$$

Among the $E$-functions, those which occur most frequently are $E_{0}, E_{1}, E_{-1}$, $E_{2}$. We shall write simply $E$ for $E_{1}$. The function $E_{0}$ vanishes for all values of its arguments, excepting simultaneous unit values, for which it takes the value 1 (Theorem I). The function $E=E_{1}$ takes the same value 1 for all values of its arguments. Among the $E$-functions, $E_{0}$ and $E$ are the only ones which are linear.

Among the $\lambda$-functions, the most important is $\lambda_{-1}$, which we shall write simply as $\lambda$. It will be noticed that

$$
\pi_{1}=\lambda_{0}=E_{0} ; \lambda_{1}=\epsilon_{1}=I_{0}=E .
$$

The function $I_{1}$ (which is equal to the product of its arguments) will be written simply $I$.

The generating series to the base $p$ of these elementary functions are easily obtained; they are

$$
\begin{aligned}
& E_{k(p)}(x)=1+k x+k x^{2}+\cdots=\frac{1+(k-1) x}{1-x} ; \\
& E_{k(p)}\left(x_{1}, x_{2}, \cdots, x_{r}\right)=\frac{k}{\left(1-x_{1}\right)\left(1-x_{2}\right) \cdots\left(1-x_{r}\right)}-(k-1) ; \\
& E_{0(p)}\left(x_{1}, x_{2}, \cdots, x_{r}\right)=1 ; \\
& E_{(p)}\left(x_{1}, x_{2}, \cdots, x_{r}\right)=\frac{1}{\left(1-x_{1}\right)\left(1-x_{2}\right) \cdots\left(1-x_{r}\right)} \\
& \lambda_{k(p)}\left(x_{1}, x_{2}, \cdots, x_{r}\right)=\prod_{i} \frac{1}{1-k x_{i}} ;
\end{aligned}
$$




$$
\begin{aligned}
\lambda_{(p)}\left(x_{1}, x_{2}, \cdots, x_{r}\right) & =\prod_{i} \frac{1}{1+x_{i}} ; \\
I_{k(p)}\left(x_{1}, x_{2}, \cdots, x_{r}\right) & =\prod_{i} \frac{1}{1-p^{k} x_{i}} ; \\
\epsilon_{k(p)}\left(x_{1}, x_{2}, \cdots, x_{r}\right) & =\prod_{i} \frac{1}{1-x_{i}^{k}} ; \\
\pi_{k(p)}\left(x_{1}, x_{2}, \cdots, x_{r}\right) & =\prod_{i} \frac{1-x_{i}^{k}}{1-x_{i}}
\end{aligned}
$$

Section II. The processes of the calculus

The calculus of multiplicative functions consists of certain processes, which while applicable to arithmetic functions in general, have the characteristic property of yielding only multiplicative functions, when performed on multiplicative functions. These processes are the following:

(1) Multiplication (including division) of functions;

(2) Convolution of arguments;

(3) Composition of functions (including inversion);

(4) Compounding of functions.

We shall consider these in turn.

1. Multiplication of functions. If $f\left(M_{1}, M_{2}, \cdots, M_{r}\right), \phi\left(M_{1}, M_{2}, \cdots, M_{r}\right)$ be multiplicative functions of the same $r$ arguments, their product

$$
f\left(M_{1}, M_{2}, \cdots, M_{r}\right) \phi\left(M_{1}, \cdots, M_{r}\right),
$$

which we shall denote by $(f \times \phi)\left(M_{1}, M_{2}, \cdots, M_{r}\right)$, is also a multiplicative function of $M_{1}, M_{2}, \cdots, M_{r}$.

If the generating series of $f, \phi$ to the base $p$ are

$$
\begin{aligned}
& f_{(p)}\left(x_{1}, x_{2}, \cdots, x_{r}\right)=\sum a_{m_{1}, m_{2}}, \cdots, m_{r} x_{1}^{m_{1}} x_{2}^{m_{2}} \cdots x_{r}^{m_{r}}, \\
& \phi_{(p)}\left(x_{1}, x_{2}, \cdots, x_{r}\right)=\sum b_{m_{1}, m_{2}}, \cdots, m_{r} x_{1}^{m_{1}} x_{2}^{m_{2}} \cdots x_{r}^{m_{r}},
\end{aligned}
$$

then the generating series of their product $f \times \phi$ is given by

$$
(f \times \phi)_{(p)}\left(x_{1}, x_{2}, \cdots, x_{r}\right)=\sum a_{m_{1}, m_{2}}, \cdots, m_{r} h_{m_{1}, m_{2}, \cdots, m_{r}} x_{1}^{m_{1}} x_{2}^{m_{2}} \cdots x_{r}^{m_{r}} .
$$

Also, if $\phi$ does not vanish for finite values of its arguments, we can define its reciprocal function $1 / \phi$ by

$$
\frac{1}{\phi}\left(M_{1}, M_{2}, \cdots, M_{r}\right)=\frac{1}{\phi\left(M_{1}, \cdots, M_{r}\right)} ;
$$


then

$$
\left(\frac{1}{\phi}\right)_{(p)}\left(x_{1}, x_{2}, \cdots, x_{r}\right)=\sum \frac{1}{b_{m_{1}, m_{2}, \cdots, m_{r}}} \cdot x_{1}^{m_{1}} x_{2}^{m_{2}} \cdots x_{r}^{m_{r}} .
$$

By taking the product of $f$ and the reciprocal function of $\phi$, we have the case of division.

We have defined the symbol $f \times \phi$ only for the case in which $f$ and $\phi$ are functions of the same $r$ arguments. We can extend the scope of the symbol by a device which will be generally useful. If $f$ is a function of $M_{1}, M_{2}, \cdots, M_{r}$ while $\phi$ is a function of $M_{1}, M_{2}, \cdots, M_{i}$ only $(i<r)$, we regard $\phi$ as a function of $M_{1}, M_{2}, \cdots, M_{r}$ which vanishes unless $M_{i+1}=M_{i+2}=\cdots=M_{r}=1$, and is then equal to $\phi\left(M_{1}, M_{2}, \cdots, M_{i}\right)$; that is, we consider $\phi\left(M_{1}, M_{2}, \cdots, M_{i}\right)$ to be the function $\phi\left(M_{1}, M_{2}, \cdots, M_{i}\right) E_{0}\left(M_{i+1}, \cdots, M_{r}\right)$. The justification for considering these to be identical is that the generating series are the same for the two functions (since the generating series of $E_{0}$ to any base is simply 1 ). With this convention, then, $f \times \phi$ is seen to be a function of $M_{1}, M_{2}, \cdots, M_{r}$, which vanishes unless $M_{i+1}=M_{i+2}=\cdots=M_{r}=1$, that is, for all practical purposes, a function of the common arguments only, of $f$ and $\phi$. In following this convention, it should not be forgotten that we are making a distinction between the functional multiplication in $f \times \phi$, and the multiplication of quantities; thus, if $f(M), \phi(N)$ are functions of different arguments, $(f \times \phi)(M, N)$ should be the same as $E_{0}(M, N)$ according to our interpretation, but $f(M) \times \phi(N)$ is not $E_{0}(M, N)$, the multiplication in the former case being functional, and in the latter, quantitative. The distinction between the two senses of multiplication will be generally evident from the fact that the arguments will appear explicitly in algebraic multiplication, while they will usually be dropped in functional multiplication.

If we take for $\phi$ the elementary function $E_{k}\left(M_{1}, M_{2}, \cdots, M_{r}\right)$ (Section I), we have

$$
\left(f \times E_{k}\right)_{(p)}\left(x_{1}, x_{2}, \cdots, x_{r}\right)=1+\sum k a_{m_{1}, m_{2}, \cdots, m_{r}} x_{1}^{m_{1}} x_{2}^{m_{2}} \cdots x_{r}^{m_{r}},
$$

where the summation on the right is for all zero and positive integral values of $m_{1}, m_{2}, \cdots, m_{r}$, with the exception of simultaneous zero values. In particular,

$$
\begin{gathered}
f \times E=f, \\
f \times E_{0}=E_{0} .
\end{gathered}
$$

Thus the functions $E, E_{0}$ behave like unity and zero, with respect to functional multiplication.

The linear functions have a special property with respect to multiplication, namely: 
THEOREM II. The product of linear functions is also a linear function.

For, if

$$
\begin{aligned}
& K_{(p)}\left(x_{1}, x_{2}, \cdots, x_{r}\right)=\prod_{i}\left(1-\alpha_{i} x_{i}\right)^{-1} \\
& L_{(p)}\left(x_{1}, x_{2}, \cdots, x_{r}\right)=\prod_{i}\left(1-\beta_{i} x_{i}\right)^{-1}
\end{aligned}
$$

then it is clear that

$$
(K \times L)_{(p)}\left(x_{1}, x_{2}, \cdots, x_{r}\right)=\prod_{i}\left(1-\alpha_{i} \beta_{i} x_{i}\right)^{-1} .
$$

In particular, the multiplicative powers of $L$, namely $L \times L, L \times L \times L$, etc., are all linear functions. Conversely, we may expect the existence of linear root-functions $L_{1}, L_{2}, \cdots$, such that

$$
L_{1} \times L_{1}=L ; L_{2} \times L_{2} \times L_{2}=L ; \cdots .
$$

Supposing, for simplicity, that $L$ is a linear function of a single argument, we can take

$$
L_{(p)}(x)=1+\alpha x+\alpha^{2} x^{2}+\cdots .
$$

Then, since $L_{1}$ is to be linear, we must have

$$
L_{1(p)}(x)= \begin{cases}\text { either } & 1+\alpha^{1 / 2} x+\left(\alpha^{1 / 2}\right)^{2} x^{2}+\cdots=\left(1-\alpha^{1 / 2} x\right)^{-1}, \\ \text { or } & 1-\alpha^{1 / 2} x+\left(-\alpha^{1 / 2}\right)^{2} x^{2}+\cdots=\left(1+\alpha^{1 / 2} x\right)^{-1}\end{cases}
$$

Thus each element of the root-function $L_{1}$ has two determinations, and therefore by choosing one of the two admissible elements for each prime base (which we are at liberty to do, from the independence of the elements of a multiplicative function), we can construct the root-function $L_{1}$ in an unenumerably infinite number of ways. This shows that the concept "rootfunction" is an unprofitable one. We shall however see later on, that we can construct a unique function called the "root-composite" from the elements of the root-function.

2. Convolution of arguments. Let $f\left(M_{1}, M_{2}, \cdots, M_{r}\right)$ be a multiplicative function of $r(>1)$ arguments. The process of convolving $M_{1}, M_{2}$ in $f$ consists in forming the function

$$
\phi\left(M, M_{3}, \cdots, M_{r}\right)=\sum_{M_{1} M_{2}=M} f\left(M_{1}, M_{2}, M_{3}, \cdots, M_{r}\right) .
$$

From the multiplicative property of $f$ it is easy to show that $\phi$ is a multiplicative function of its $r-1$ arguments.

If the generating series of $f$ to the base $p$ be 


$$
f_{(p)}\left(x_{1}, x_{2}, \cdots, x_{r}\right)=\sum a_{m_{1}, m_{2}}, \cdots, m_{r} x_{1}^{m_{1}} x_{2}^{m_{2}} \cdots x_{r}^{m_{r}}
$$

then

$$
\phi_{(p)}\left(x, x_{3}, \cdots, x_{r}\right)=\sum\left(\sum_{m_{1}+m_{2}=m} a_{m_{1}, m_{2}}, \cdots, m_{r}\right) x^{m^{m} x_{3}^{m_{3}} \cdots x_{r}^{m_{r}}} .
$$

Thus the generating series $\phi_{(p)}\left(x, x_{3}, \cdots, x_{\tau}\right)$ is obtained by putting $x_{1}=x_{2}=x$ in $f_{(p)}\left(x_{1}, x_{2}, \cdots, x_{r}\right)$. Therefore,

ThEOREM III. Convolution of arguments is equivalent to identifying the corresponding variables in the generating series.

The process of convolution is evidently applicable to any number $s$ of arguments, and is equivalent to the identification of the $s$ corresponding variables in the generating series. Hence in convolving several arguments, it is immaterial whether we convolve them all together, or in stages.

The functions obtained by convolving sets of arguments in all possible ways in $f\left(M_{1}, M_{2}, \cdots, M_{r}\right)$ may be called convolutes of $f$. Among these convolutes, there is one which is a function of a single argument only, namely, that in which all the arguments have been convolved together. This particular convolute may be termed "the convolute of $f$."

We shall also use the term convolute in another special sense. If $\psi(M)$ is a multiplicative function of a single argument, we can define a multiplicative function $\psi^{\prime}\left(M_{1}, M_{2}, \cdots, M_{r}\right)$ of $r$ arguments by

$$
\psi^{\prime} \quad(M, M, \cdots, M)=\psi(M),
$$

$\psi^{\prime}\left(M_{1}, M_{2}, \cdots, M_{r}\right)=0$, if two of the arguments are unequal.

The function $\psi^{\prime}$ is termed the principal function of $r$ arguments equivalent to $\psi(M)$. The convolute $\psi_{r}(M)$ of $\psi^{\prime}\left(M_{1}, M_{2}, \cdots, M_{r}\right)$ will be referred to as the rth convolute of $\psi(M)$.

Theorem IV. The rth convolute $\psi_{r}(M)$ of $\psi(M)$ is the function defined by

$$
\psi_{r}(M)=\left\{\begin{array}{l}
\psi\left(M^{1 / r}\right), \text { if } M \text { is an } r \text { th power, } \\
0, \text { otherwise. }
\end{array}\right.
$$

For, by the definition,

$$
\psi_{r}(M)=\sum \psi^{\prime}\left(M_{1}, M_{2}, \cdots, M_{r}\right),
$$

where $\psi^{\prime}$ is the principal function of $r$ arguments equivalent to $\psi(M)$, and the summation on the right is for all values of $M_{1}, M_{2}, \cdots, M_{r}$ such that $M_{1} M_{2} \cdots M_{r}=M$. Since $\psi^{\prime}$ vanishes whenever two of its arguments are unequal, it follows that the right side vanishes when $M$ is not an $r$ th power, and is equal to $\psi^{\prime}(N, N, \cdots, N)=\psi(N)$, if $M=N^{r}$. As an illustration, the 
elementary function $\epsilon_{k}$ is the $k$ th convolute of $E$. It is obvious that the generating series of the $r$ th convolute of $\psi(M)$ is obtained by substituting $x^{r}$ for $x$, in the corresponding generating series of $\psi$.

To avoid misunderstanding, we shall use the phrase $r$ th convolute of $\psi$ with the second meaning of convolute, only for functions $\psi$ of a single argument. The great utility of this concept will be seen from the applications in II $\$ 5$ (c).

3. Composition of functions. Let $f_{1}\left(M_{1}, M_{2}, \cdots, M_{r}\right), f_{2}\left(N_{1}, N_{2}, \cdots, N_{r}\right)$ be two multiplicative functions of $r$ arguments. Then $f_{1}\left(M_{1}, M_{2}, \cdots, M_{r}\right)$ $\times f_{2}\left(N_{1}, N_{2}, \cdots, N_{r}\right)$ is a multiplicative function of $2 r$ arguments. The result of convolving the $r$ pairs of corresponding arguments $\left(M_{i}, N_{i}\right)$ in this product is therefore a multiplicative function $f$ of $r$ arguments. We call $f$ the composite of $f_{1}, f_{2}$, and denote it by the functional symbol $\left(f_{1} \cdot f_{2}\right)$.

From the arithmetical significance of convolution, it follows that the composite can be defined arithmetically by

$$
f\left(M_{1}, M_{2}, \cdots, M_{r}\right)=\sum f_{1}\left(\delta_{1}, \delta_{2}, \cdots, \delta_{r}\right) f_{2}\left(\frac{M_{1}}{\delta_{1}}, \frac{M_{2}}{\delta_{2}}, \ldots, \frac{M_{r}}{\delta_{r}}\right),
$$

summed for all divisors $\delta_{i}$ of $M_{i}(i=1,2, \cdots, r)$.

There is a simple relation between the generating series of $f_{1}, f_{2}$ and their composite $f$. If $f_{1(p)}\left(x_{1}, x_{2}, \cdots, x_{r}\right), f_{2(p)}\left(y_{1}, y_{2}, \cdots, y_{r}\right)$ are the generating series of $f_{1}, f_{2}$ to the base $p$, the generating series of $f_{1}\left(M_{1}, M_{2}, \cdots, M_{r}\right)$ $\times f_{2}\left(N_{1}, N_{2}, \cdots, N_{r}\right)$ to the same base is evidently $f_{1(p)}\left(x_{1}, x_{2}, \cdots, x_{r}\right)$ $\times f_{2(p)}\left(y_{1}, y_{2}, \cdots, y_{r}\right)$. Since convolution of arguments is equivalent to identification of corresponding variables in the generating series (Theorem III), it follows that the generating series of the composite is given by

$$
f_{(p)}\left(z_{1}, z_{2}, \cdots, z_{r}\right)=f_{1(p)}\left(z_{1}, z_{2}, \cdots, z_{r}\right) \times f_{2(p)}\left(z_{1}, z_{2}, \cdots, z_{r}\right) .
$$

Thus,

THEOREM V. Composition of functions of $r$ arguments is equivalent to the multiplication of their generating series to each base, after identifying corresponding variables.

Hence composition of several functions of $r$ arguments is associative and commutative.

The process of composition of $f_{1}, f_{2}$ implies a correspondence between their arguments. A convenient way of expressing this fact would be to say that $f\left(M_{1}, M_{2}, \cdots, M_{r}\right)$ is the composite of the functions $f_{1}, f_{2}$ of the same $r$ arguments $M_{1}, M_{2}, \cdots, M_{r}$. With this understanding we can interpret the composite of functions of different arguments in the same way as was done in the 
case of multiplication. Thus the composite of $\psi\left(M_{1}\right)$ and $f\left(M_{1}, M_{2}, \cdots, M_{r}\right)$ is to be interpreted to mean the composite of $\psi\left(M_{1}\right) E_{0}\left(M_{2}, \cdots, M_{r}\right)$ and $f\left(M_{1}, M_{2}, \cdots, M_{r}\right)$, which are functions of the same $r$ arguments. In the same way, the composite of functions $f_{1}\left(M_{1}, M_{2}, \cdots, M_{r}\right), f_{2}\left(N_{1}, N_{2}, \cdots, N_{r}\right)$ without common arguments is to be interpreted as the composite of

$$
f_{1}\left(M_{1}, M_{2}, \cdots, M_{r}\right) E_{0}\left(N_{1}, N_{2}, \cdots, N_{r}\right)
$$

and $E_{0}\left(M_{1}, M_{2}, \cdots, M_{r}\right) f_{2}\left(N_{1}, N_{2}, \cdots, N_{r}\right)$, and is easily seen to reduce to the product $f_{1}\left(M_{1}, M_{2}, \cdots, M_{r}\right) \times f_{2}\left(N_{1}, N_{2}, \cdots, N_{r}\right)$. Theorem V is easily seen to hold when composition is extended in this manner.*

The composites of $f\left(M_{1}, M_{2}, \cdots, M_{r}\right)$ with itself will be indicated by exponents. Thus $f \cdot f=f^{2} ; f \cdot f \cdot f=f^{3}$; etc. To avoid confusion, products like $f \times f$ will not be denoted by exponents, but will be written out in full.

The composite of $f\left(M_{1}, M_{2}, \cdots, M_{r}\right)$ and $E\left(M_{1}, M_{2}, \cdots, M_{r}\right)$ is given by

$$
(f \cdot E)\left(M_{1}, M_{2}, \cdots, M_{r}\right)=\sum f\left(\delta_{1}, \delta_{2}, \cdots, \delta_{r}\right)
$$

* The object in introducing the extended sense of composition as well as functional multiplication in addition to quantitative multiplication may be explained here. Composition in the unextended sense has two defects; namely

(1) The symbol $f_{1} \cdot f_{2}$ is not completely defined by $f_{1}$ and $f_{2}$, but requires in addition a knowledge of the correspondence between the arguments in $f_{1}, f_{2}$.

(2) Since composition has been defined only for functions of the same number of arguments, we cannot speak of an expression like $\sum \psi(\delta) f(M / \delta, N)$ summed for divisors $\delta$ of $M$, as a composite.

Both these defects are removed if we adopt the following conventions:

(1) Corresponding arguments in the composition $f_{1} \cdot f_{2}$ shall be thought of as identical, so that composition becomes a process which is defined (in the first instance) only for two functions of the same $r$ arguments.

(2) If $f_{1}, f_{2}$ are not functions of the same arguments, they are converted into functions $F_{1}, F_{2}$ of the same arguments, by multiplication by $E_{0}$ (as explained in the text), and $f_{1} \cdot f_{2}$ is defined to be $F_{1} \cdot F_{2}$.

If we accept these conventions, it follows that between any two functional symbols $f_{1}, f_{2}$ there exists a relation, which is expressed by one of the three statements " $f_{1}$ and $f_{2}$ are functions of the same arguments, or $f_{1}$ and $f_{2}$ have some or no common arguments." Does this relation between $f_{1}, f_{2}$, which, by our accepting the conventions, has become implicit in the functional symbols themselves, affect the product $f_{1} \times f_{2}$ ? The answer is: $f_{1} \times f_{2}$ must be interpreted as the functional product, and this is the only possible answer if we wish Theorem VII (namely $\theta \times\left(f_{1} \cdot f_{2}\right)=\left(\theta \times f_{1}\right) \cdot\left(\theta \times f_{2}\right)$, when $\theta$ is linear) to be unconditionally true. For example $\theta(M) \times\left(f_{1}(M) \cdot f_{2}(N)\right)$ is equal to $\theta(M) \times\left(f_{1}(M) f_{2}(N)\right)$ (by the use of the extended definition of composition), which cannot be equal to $\left(\theta(M) \times f_{1}(M)\right) \cdot(\theta(M) \times f(N))$, unless we interpret $\times$ as functional multiplication, in which case we should have

$$
\begin{aligned}
\theta(M) \times\left(f_{1}(M) f_{2}(N)\right) & =\theta(M) f_{1}(M) E_{0}(N), \\
\theta(M) \times f(N) & =E_{0}(M, N) \\
\left(\theta(M) \times f_{1}(M)\right) \cdot(\theta(M) \times f(N)) & =\left\{\theta(M) f_{1}(M) E_{0}(N)\right\} \cdot E_{0}(M, N)=\theta(M) f_{1}(M) E_{0}(N) .
\end{aligned}
$$

Even without this example, functional multiplication and extended composition may be justified by using generating series, as the reader may easily verify for himself.

The reader may note the elegant application of extended composition, made in the proof of Theorem $\mathrm{X}$ below. 
summed for all divisors $\delta_{i}$ of $M_{i}(i=1,2, \cdots, r)$. We call $f \cdot E$ the numerical integral or simply the integral of $f$. The function $E^{2}$ is the integral of $E$, and is given by

$$
\begin{gathered}
E^{2}(M)=\text { number of divisors of } M \\
E^{2}\left(M_{1}, M_{2}, \cdots, M_{r}\right)=E^{2}\left(M_{1}\right) E^{2}\left(M_{2}\right) \cdots E^{2}\left(M_{r}\right) .
\end{gathered}
$$

The function $E^{k}(M)$, where $k$ is a positive integer, is equal, from the definition of composition, to the number of ways of expressing $M$ as a product of $r$ factors, attention being paid to the order of the factors.

The composite of $f_{1}, f_{2}$ has been defined as the result of convolving the $r$ pairs $M_{i}, N_{i}$ in the product $f_{1}\left(M_{1}, M_{2}, \cdots, M_{r}\right) \times f_{2}\left(N_{1}, N_{2}, \cdots, N_{r}\right)$. Now; we have seen that the result of a series of convolutions is independent of the order in which they are performed. Hence the result of performing any convolution on the composite of $f_{1}, f_{2}$ is the same as that of first performing this convolution on $f_{1}$ and $f_{2}$, and then taking their composite. Hence

THEOREM VI. Convolution is distributed by composition; or, explicitly, $\Omega\left(f_{1} \cdot f_{2}\right)=\left(\Omega f_{1}\right) \cdot\left(\Omega f_{2}\right)$, where $f_{1}, f_{2}$ are functions of $r$ arguments, and $\Omega$ represents any convolution of the arguments.

In particular the rth convolute of the composite of $f(M), \phi(M)$ is the composite of their rth convolutes.

We have already observed that, given a linear function $L$, we can always find a linear function $L^{\prime}$, such that $L^{\prime} \times L^{\prime} \times \cdots$ (to $r$ factors) $=L$, and that each element of $L^{\prime}$ has $r$ determinations. Therefore we can find $r$ linear functions $L_{i}(i=1,2, \cdots, r)$ no two of which have any elements in common, such that

$$
L_{i} \times L_{i} \times \cdots(r \text { factors })=L, \quad i=1,2, \cdots, r .
$$

It is clear that the elements of $L_{i}$ to any base $p$ are the $r$ determinations of the elements of $L^{\prime}$ to the same base. Hence, even though the functions $L_{i}$ can be chosen in an unenumerably infinite number of ways, yet their continued composite $L_{1} \cdot L_{2} \cdot L_{3} \cdots L_{r}$ is the same function $f$ in every case; we call $f$ the rth root-composite of the linear function $L$. An important property of the linear function is

THEOREM VI(a). The rth root-composite of a linear function is also its rth convolute.

For, if

$$
L_{(p)}(x)=\frac{1}{1-a x}
$$

then 


$$
f_{(p)}(x)=\frac{1}{\left(1-a^{1 / r} x\right)\left(1-\omega a^{1 / r} x\right) \cdots\left(1-\omega^{r-1} a^{1 / r} x\right)}=\frac{1}{1-a x^{r}},
$$

$\omega$ being a primitive $r$ th root of unity. But $1 /\left(1-a x^{r}\right)$ is clearly the generating series, to the base $p$, of the $r$ th convolute of $L$; hence the theorem.

The function $E_{0}$ plays a special rôle in composition. For, since its generating series to any base is 1 , we must have

$$
f \cdot E_{0}=f ; E_{0}^{k}=E_{0} .
$$

It was observed that $E_{0}$ behaves like zero with respect to functional multiplication. Accepting the analogy, the fact that $f \cdot E_{0}=f$ suggests that composition must be considered analogous to addition. Like addition, composition is associative and commutative, but unlike addition it does not distribute (functional) multiplication. It has however a restricted distributive property given by

THEOREM VII. The compositional operation distributes multiplication, whenever the multiplier is a linear function.

For, let $\theta\left(M_{1}, M_{2}, \cdots, M_{r}\right)$ be a linear function multiplying the composite $(f \cdot \phi)\left(M_{1}, M_{2}, \cdots, M_{r}\right)$. We have

$\{(\theta \times f) \cdot(\theta \times \phi)\}\left(M_{1}, M_{2}, \cdots, M_{r}\right)$

$=\sum \theta\left(\delta_{1}, \delta_{2}, \cdots, \delta_{r}\right) f\left(\delta_{1}, \delta_{2}, \cdots, \delta_{r}\right) \theta\left(\frac{M_{1}}{\delta_{1}}, \frac{M_{2}}{\delta_{2}}, \ldots, \frac{M_{r}}{\delta_{r}}\right) \phi\left(\frac{M_{1}}{\delta_{1}}, \ldots, \frac{M_{r}}{\delta_{r}}\right)$

$=\sum \theta\left(M_{1}, M_{2}, \cdots, M_{r}\right) f\left(\delta_{1}, \cdots, \delta_{r}\right) \phi\left(\frac{M_{1}}{\delta_{1}}, \ldots, \frac{M_{r}}{\delta_{r}}\right)$, since $\theta$ is linear,

$=\{\theta \times(f \cdot \phi)\}\left(M_{1}, M_{2}, \cdots, M_{r}\right)$.

It is easy to see that the same result will hold even if some of the arguments in $\theta$ are different from those in the composite.

4. Inversion. From each generating series $f_{(p)}\left(x_{1}, x_{2}, \cdots, x_{r}\right)$ of a multiplicative function $f\left(M_{1}, M_{2}, \cdots, M_{r}\right)$, we can determine uniquely a second power series $f_{(p)}^{-1}\left(x_{1}, x_{2}, \cdots, x_{r}\right)$ such that, on term-by-term multiplication, we get

$$
f_{(p)}\left(x_{1}, x_{2}, \cdots, x_{r}\right) \times f_{(p)}^{-1}\left(x_{1}, x_{2}, \cdots, x_{r}\right)=1
$$

(apart from any questions of convergence which are irrelevant). The series $f_{(p)}^{-1}$ have all the constant term unity, and are therefore the generating series of a determinate multiplicative function $f^{-1}$, which we call the inverse of $f$. The relation between the functions $f, f^{-1}$ is clearly symmetrical, each being the inverse of the other. 
Since every generating series of $E_{0}\left(M_{1}, M_{2}, \cdots, M_{r}\right)$ is 1 , the composite of $f$ and its inverse function $f^{-1}$ is $E_{0}$. This property may be taken as the definition of the inverse function. It also follows from this that in a compositional equation we can transpose any term from one side to the other, provided we replace it by its inverse. Thus from

we have

$$
f_{1} \cdot f_{2}=\phi_{1} \cdot \phi_{2},
$$

$$
\phi_{1}^{-1} \cdot f_{1} \cdot f_{2}=\phi_{1}^{-1} \cdot \phi_{1}: \phi_{2}=E_{0} \cdot \phi_{2}=\phi_{2} \text {. }
$$

The only function identical with its inverse is the function $E_{0}$. Since $E(M)$ has the generating function $1 /(1-x)$ to any base, the generating function of $E^{-1}(M)$ to any base is $1-x$; hence $E^{-1}(M)$ is the same as Mertens' function $\mu(M)$ which is equal to zero if $M$ has a squared factor, and to $(-1)^{\nu}$ if $M$ is the product of $\nu$ different primes. The function $E^{-1}\left(M_{1}, M_{2}, \cdots, M_{r}\right)$ is equal to $E^{-1}\left(M_{1}\right) E^{-1}\left(M_{2}\right) \cdots E^{-1}\left(M_{r}\right)$.

THEOREM VIII. Inversion is permutable with convolution.

For the equation $f_{(p)}\left(x_{1}, x_{2}, \cdots, x_{r}\right) \times f_{(p)}^{-1}\left(x_{1}, x_{2}, \cdots, x_{r}\right)=1$ continues to be true if we put $x_{1}=x_{2}=x$. Hence if $\Omega$ represents any convolution or series of convolutions of the arguments, $\Omega\left(f^{-1}\right)$ must be identical with $(\Omega f)^{-1}$. It also follows from this theorem, that the inverse of the $r$ th convolute of $f(M)$ is the $r$ th convolute of its inverse.

THEOREM IX. Inversion is distributed by composition; that is, the inverse of the composite of any number of functions is identical with the composite of their inverses.

For, the generating series to base $p$ of the inverse of the composite of $f, f^{\prime}$ is

$$
\left\{f_{(p)}\left(x_{1}, x_{2}, \cdots, x_{r}\right) \times f_{(p)}^{\prime}\left(x_{1}, x_{2}, \cdots, x_{r}\right)\right\}^{-1},
$$

while the generating series to the same base of the composite of their inverses is

$$
\left\{f_{(p)}\left(x_{1}, x_{2}, \cdots, x_{r}\right)^{-1} \times f_{(p)}^{\prime}\left(x_{1}, x_{2}, \cdots, x_{r}\right)^{-1}\right\} .
$$

Since these series are the same, the theorem follows.

Corollary. It follows from this theorem that $\left(f^{-1}\right)^{r}=\left(f^{r}\right)^{-1}$. We may accordingly denote each of these by $f^{-r}$, so that

$$
\begin{aligned}
f^{-r} & =\left(f^{-1}\right)^{r}=\left(f^{r}\right)^{-1}, \\
f^{r+*} & =f^{r} \cdot f^{s}, \text { for positive or negative integers } r, s ; \\
f^{0} & =E_{0} .
\end{aligned}
$$


It was already observed that the product of two functions

$$
f_{1}\left(M_{1}, M_{2}, \cdots, M_{r}\right), f_{2}\left(N_{1}, N_{2}, \cdots, N_{r}\right)
$$

without common arguments is the composite of the two functions $f_{1}\left(M_{1}, M_{2}, \cdots, M_{r}\right) E_{0}\left(N_{1}, N_{2}, \cdots, N_{r}\right)$ and $E_{0}\left(M_{1}, M_{2}, \cdots, M_{r}\right) f_{2}\left(N_{1}\right.$, $\left.N_{2}, \cdots, N_{r}\right)$. Now, it is easily seen that the inverse of $f_{1}\left(M_{1}, M_{2}, \cdots\right.$, $\left.M_{r}\right) E_{0}\left(N_{1}, \cdots, N_{r}\right)$ is $f_{1}^{-1}\left(M_{1}, M_{2}, \cdots, M_{r}\right) E_{0}\left(N_{1}, \cdots, N_{r}\right)$. Hence, from Theorem IX we have

THEOREM X. The inverse of the product of functions without common arguments is the product of their inverses.

An important property of the linear functions with respect to inversion is given by

THEOREM XI. If $\theta$ is a linear, and $f$ an arbitrary, function, the inverse of $\theta \times f$ is $\theta \times f^{-1}$.

For

$$
\begin{aligned}
(\theta \times f) \cdot\left(\theta \times f^{-1}\right) & \left.=\theta \times\left(f \cdot f^{-1}\right) \quad \text { (Theorem VII }\right) \\
& =\theta \times E_{0} \\
& =E_{0} .
\end{aligned}
$$

Corollary. Since $\theta=\theta \times E$, the inverse of $\theta$ is $\theta \times E^{-1}$.

5. Some applications to functions of a single argument. (a) Examples. The following functions of the argument $N$ are of frequent occurrence:

(1) $\tau(N)=$ number of divisors of $N=E^{2}(N) ; \sigma(N)=$ sum of the divisors of $N=(I \cdot E)(N) ; \sigma_{k}(N)=$ sum of the $k$ th powers of the divisors of $N=\left(I_{k} \cdot E\right)(N)$; $\mu(N)=E^{-1}(N)$.

(2) $\phi(N)=$ Euler's function representing the number of numbers less than and prime to $N=\left(I \cdot E^{-1}\right)(N) ; \phi_{k}(N)=$ Jordan's function* representing the number of sets of $k$ numbers not greater than $N$, whose greatest common divisor is prime to $N,=\left(I_{k} \cdot E^{-1}\right)(N) ; \tau_{k}(N)=\tau\left(N^{k}\right)=\left(E_{k} \cdot E\right)(N)$.

(3) $I_{r, s}(N)=N^{r}$, if $N$ is an sth power, and $=0$, otherwise; in other words $I_{r, s}$ is the sth convolute of $I_{r}$.

To prove the statements in (2), we note that the number of numbers not greater than $N$ which have the greatest common divisor $\delta$ with it, is $\phi(N / \delta)$. Hence

$$
\sum_{\delta / N} \phi\left(\frac{N}{\delta}\right)=N,
$$

* See Dickson's History of the Theory of Numbers, vol. 1, p. 147. This book will be hereafter quoted by the author's name. 
or $\phi \cdot E=I$, so that $\phi=I \cdot E^{-1}$. An exactly similar type of grouping shows that $\phi_{k}=I_{k} \cdot E^{-1}$, and also proves Cesàro's general result*

$$
\sum_{j=1}^{N} f\left(d_{j}\right)=(f \cdot \phi)(N)
$$

where $d_{j}$ denotes the greatest common divisor of $j$ and $N$.

To prove that $\tau_{k}=E_{k} \cdot E$, we note that $E_{k}(N)$ is equal to the number of divisors of $N^{k}$ which do not divide $\delta^{k}$, where $\delta(<N)$ is any divisor of $N$. For, if $N=p^{\alpha^{1}} p_{2}^{\alpha} \cdots p_{\nu}^{\alpha}$, the divisors in question are the terms of the product

$$
\left(p_{1}^{\left(\alpha_{1}-1\right) k+1}+p_{1}^{\left(\alpha_{1}-1\right) k+2}+\cdots+p^{\alpha_{1} k}\right)\left(p_{2}^{\left(\alpha_{2}-1\right) k+1}+\cdots+p_{2}^{\alpha_{2} k}\right),
$$

and are hence $k^{\nu}$ in number. Now to each divisor $D$ of $N^{k}$ we can make correspond a unique divisor $\delta$ of $N$, such that $D$ divides $\delta^{k}$, but does not divide the $k$ th power of any other divisor of $\delta$. Hence $\left(E_{k} \cdot E\right)(N)$ enumerates all the divisors of $N^{k}$, or $\tau_{k}=E_{k} \cdot E$.

As applications of Theorem VII, we have

Example 1. If $L$ is a linear function, $L^{k}=L \times E^{k}$, where $k$ is a positive or negative integer. For

$$
\begin{aligned}
L^{k}=L \cdot L \cdot L \cdots & =(L \times E) \cdot(L \times E) \cdots \\
& =L \times(E \cdot E \cdots)(\text { Theorem VII })=L \times E^{k} .
\end{aligned}
$$

For the case of a negative integer, $-k$,

Hence

$$
\begin{aligned}
\left(L \times E^{k}\right) \cdot\left(L \times E^{-k}\right) & =L \times\left(E^{k} \cdot E^{-k}\right) \text { (Theorem VII) } \\
& =L \times E_{0}=E_{0} .
\end{aligned}
$$

$$
L^{-k}=\left(L^{k}\right)^{-1}=\left(L \times E^{k}\right)^{-1}=L \times E^{-k} \text { (Theorem XI). }
$$

As an example, or, explicitly,

$$
\sigma \cdot \sigma=I \cdot E \cdot I \cdot E=\left(I \times E^{2}\right) \cdot E^{2},
$$

$$
\sum_{\delta / N} \sigma(\delta) \sigma\left(\frac{N}{\delta}\right)=\sum_{\delta / N} \delta \tau(\delta) \tau\left(\frac{N}{\delta}\right) \text { (Liouville) } \dagger
$$

Example 2.

$$
\left.\sum_{\delta / N} \delta^{\mu-\nu} \sigma_{\nu+\lambda}(\delta) \sigma_{\mu+\rho}\left(\frac{N}{\delta}\right)=\sum_{\delta / N} \delta^{\mu-v} \sigma_{\nu+\rho}(\delta) \sigma_{\mu+\lambda}\left(\frac{N}{\delta}\right) \text { (Liouville) }\right) .
$$

* Dickson, p. 127, Note 57.

† Dickson, p. 285, 25.

† Dickson, p. 286, 30 . 
For the left side

$$
\begin{aligned}
& =\left\{I_{\mu-\nu} \times\left(I_{\nu+\lambda} \cdot E\right)\right\} \cdot I_{\mu+\rho} \cdot E \\
& =I_{\mu+\lambda} \cdot I_{\mu-\nu} \cdot I_{\mu+\rho} \cdot E \text { (Theorem VII), }
\end{aligned}
$$

and the symmetry of this in $\lambda, \rho$ proves the result.

(b) Relations between the elementary functions. The following relations between the elementary functions are fundamental:

$$
\begin{aligned}
\boldsymbol{\epsilon}_{k} \cdot \pi_{k} & =E ; \\
E_{1-k} & =E \cdot \lambda_{k}^{-1} ;
\end{aligned}
$$

in particular,

$$
\begin{gathered}
E_{2} \cdot \lambda=E ; \\
E \cdot \lambda=\epsilon_{2} ; \\
E_{2} \cdot \epsilon_{2}=E^{2} ; \\
E_{2} \times \lambda=E_{2}^{-1} .
\end{gathered}
$$

These relations are immediately evident from the generating series and are also capable of direct arithmetical proof. For instance the truth of (1) follows from the fact that a number can be expressed in only one way as the product of two factors, the first of which is an exact $k$ th power, and the second is not divisible by any $k$ th power. To prove (3), we observe that $E \cdot \lambda^{-1}(N)$ enumerates all the divisors of $N$ which possess no squared factor, and is therefore equal to $E_{2}(N)$. The relation (4) expresses the fact that the second convolute $\epsilon_{2}$ of $E$ is also its second root-composite (Theorem VI (a)); for, the linear functions $E, \lambda$ have no common elements, and each of them yields $E$ on multiplication with itself, and therefore the second root-composite of $E$ is $E \cdot \lambda$. To prove (5), we note that the number of divisor-pairs $\delta, N / \delta$ of $N$ with a given greatest common divisor $\delta_{1}$ is zero if $\delta_{1}{ }^{2}$ does not divide $N$, and $E_{2}\left(N / \delta_{1}{ }^{2}\right)$ otherwise. Hence $\left(E_{2} \cdot \epsilon_{2}\right)(N)$ is equal to the total number of divisors of $N$. The relation (6) is a consequence of (3); for, by (3),

therefore

$$
E_{2}=E \cdot \lambda^{-1}
$$

$$
\begin{aligned}
E_{2} \times \lambda=\lambda \times\left(E \cdot \lambda^{-1}\right) & =\lambda \cdot\left(\lambda \times \lambda^{-1}\right) \text { (Theorem VII) } \\
& =\lambda \cdot E^{-1}=E_{2}^{-1} \text { (Theorem IX) }
\end{aligned}
$$

Example 3. $E_{2} \cdot E^{2}=E^{2} \times E^{2}$. For $\left(E^{2} \times E^{2}\right)(N)$ is the number of divisorpairs of $N$. Let $\delta_{1}, \delta_{2}$ be two divisors of $N, l$ their least common multiple, and $\boldsymbol{t}$ their greatest common divisor. Group the divisor-pairs in such a way that, for each group, $l / t$ is a fixed divisor $\delta$ of $N$. It is clear that, for each group, $t$ 
is an arbitrary divisor of $N / \delta$, while $\delta_{1}, \delta_{2}$ must be of the form $t l_{1}, t l_{2}$, where $l_{1}, l_{2}$ are relatively prime, and $l_{1} l_{2}=\delta$. Thus the number of divisor-pairs in the group specified by $\delta$ is $\tau(N / \delta) E_{2}(\delta)$. Thus $E_{2} \cdot E^{2}=E^{2} \times E^{2}$.

As an alternative proof, the number of divisor-pairs of $N$, which have $\delta$ for their greatest common divisor, and $N$ for their least common multiple, is $E_{2}(N / \delta)$. Hence $\left(E_{2} \cdot E\right)(N)$ is the number of factor-pairs which have $N$ for their least common multiple. Hence $E_{2} \cdot E \cdot E=\left(E_{2} \cdot E^{2}\right)(N)$ is equal to the total number of factor-pairs of $N$, that is, to $\left(E^{2} \times E^{2}\right)(N)$.

The following results bear on this and previous theorems:

$$
\begin{aligned}
(\tau \times \tau) \cdot\left(E_{2} \times \lambda\right)=\left(E^{2} \times E^{2}\right) \cdot E_{2}^{-1}=E^{2}, \text { or } \\
\left.\sum\{\tau(\delta)\}^{-2} \lambda\left(\frac{N}{\delta}\right) E_{2}\left(\frac{N}{\delta}\right)=\tau(N) \text { (Liouville }\right)^{*} ; \\
\left.\left(\lambda \times \tau_{2}\right) \cdot \sigma_{\mu}=\left(\lambda \times E_{2}\right) \cdot \lambda \cdot E \cdot I_{\mu} \text { (Theorem VII }\right) \\
=E_{2}^{-1} \cdot \lambda \cdot E \cdot I_{\mu}=\lambda \cdot \lambda \cdot I_{\mu}=\left(\lambda \times E^{2}\right) \cdot I_{\mu} ; \text { or } \\
\sum \lambda(\delta) \tau\left(\delta^{2}\right) \sigma_{\mu}\left(\frac{N}{\delta}\right)=\sum \lambda(\delta) \tau(\delta)\left(\frac{N}{\delta}\right)^{\mu}(\text { Liouville }) \dagger ; \\
\tau_{2} \cdot \lambda=E_{2} \cdot E \cdot \lambda=E^{2}, \text { or } \\
\sum \tau\left(\delta^{2}\right) \lambda(N / \delta)=\tau(N) \text { (Liouville). } \ddagger
\end{aligned}
$$

Example 4. $\sum \tau\left(\delta_{1}\right) \tau\left(\delta_{2}\right)$ summed for all pairs of numbers $\delta_{1}, \delta_{2}$ with the least common multiple $N$ is equal to $\{\tau(N)\}^{3}$.

To prove this, let us introduce the term "block-factor of $N$ " to denote a factor $\delta$ in which each prime factor has the same exponent as in $N$, that is, a factor $\delta$ which is relatively prime to $N / \delta$. Two factors $\delta_{1}, \delta_{2}$ which have $N$ for their least common multiple, can be evidently put into the form

$$
\begin{aligned}
& \delta_{1}=P Q r, \\
& \delta_{2}=P R q,
\end{aligned}
$$

where $P, Q, R$ are block-factors such that $P Q R=N$, and $q, r$ are respectively factors of $Q, R$, having no common block-factors with them. Hence

$$
\begin{aligned}
\tau\left(\delta_{1}\right) \tau\left(\delta_{2}\right) & =\tau(P) \tau(Q) \tau(r) \tau(p) \tau(R) \tau(q) \\
& =\tau(N) \tau(P) \tau(q) \tau(r) .
\end{aligned}
$$

Thus $\sum \tau\left(\delta_{1}\right) \tau\left(\delta_{2}\right)$ is of the form $\tau(N) \sum \tau(\delta)$, where each $\delta$ occurs as many times as $N / \delta$ can be expressed as the product of relatively prime factors. That is, $\sum \tau\left(\delta_{1}\right) \tau\left(\delta_{2}\right)=\tau \times\left(\tau \cdot E_{2}\right)=E^{2} \times E^{2} \times E^{2}$ (Example 3).

* Dickson, p. 285, 27.

$\dagger$ Dickson, p. 286, 29.

‡ Dickson, p. 285, 27. 
If we make $N$ vary over the factors of a given number $M$, and sum each side of this result, we obtain Liouville's theorem:*

$$
\left\{\sum_{\delta / M} \tau(\delta)\right\}^{2}=\sum_{\delta / M}\{\tau(\delta)\}^{3}, \text { or } E^{3} \times E^{3}=\left(E^{2} \times E^{2} \times E^{2}\right) \cdot E .
$$

(c) Applications of Theorem VI. The following results due to Liouville and Gegenbauer serve as applications of the theorem that the $r$ th convolute of a composite is the composite of the $r$ th convolutes:

$$
\begin{aligned}
\sum \phi(D) \tau\left(\frac{N}{D^{2}}\right) & =\sum D E_{2}\left(\frac{N}{D^{2}}\right) ; \\
\sum E_{2}(D) \tau\left(\frac{N}{D^{2}}\right) & =\sum \tau\left(D^{2}\right) E_{2}\left(\frac{N}{D^{2}}\right) ; \\
\sum \lambda(D) \tau\left(\frac{N}{D^{2}}\right) & =\sum E_{2}\left(\frac{N}{e^{4}}\right)
\end{aligned}
$$

where the summations extend over the square divisors $D^{2}$ and the biquadrate divisors $e^{t}$ of $N$.

Writing conv $f$ and conv $r f$ for the second and the $r$ th convolutes of $f$, we have

$$
\begin{aligned}
\operatorname{conv} \phi \cdot \tau & =\operatorname{conv} I \cdot \operatorname{conv} E^{-1} \cdot \tau \\
& =\operatorname{conv} I \cdot \epsilon_{2}^{-1} \cdot E^{2} \\
& =\operatorname{conv} I \cdot E_{2}
\end{aligned}
$$

which proves (1);

(2) $\operatorname{conv} E_{2} \cdot \tau=\operatorname{conv} E_{2} \cdot \epsilon_{2} \cdot E_{2}$

$$
\begin{aligned}
& =\operatorname{conv} E_{2} \cdot \operatorname{conv} E \cdot E_{2} \\
& =\operatorname{conv}\left(E_{2} \cdot E\right) \cdot E_{2} \\
& =\operatorname{conv} \tau_{2} \cdot E_{2}, \text { which proves }(2) ;
\end{aligned}
$$

(3) $\operatorname{conv} \lambda \cdot E^{2}=\operatorname{conv} \lambda \cdot \epsilon_{2} \cdot E_{2}$

$$
=\operatorname{conv}(E \cdot \lambda) \cdot E_{2}=\operatorname{conv} \epsilon_{2} \cdot E_{2}=\epsilon_{4} \cdot E_{2} \text {, which proves (3). }
$$

In what follows, $d_{t}$ denotes a divisor of $N$ such that $N / d_{t}$ is a $t$ th power, $d$ denotes an arbitrary divisor of $N, \rho_{k, t}(N)=\sum d_{t}{ }^{k}$, and the summation is for integers $m, n$ such that $N=m n^{p}$.

$$
\sum \sigma_{k}(m) \rho_{0,2}(n)=\sum \rho_{0,2 t}(d) \rho_{k, t}\left(\frac{N}{d}\right) . \ddagger
$$

* Dickson, p. 286, 28.

† These results are taken from Dickson, p. 285, 27.

$\ddagger$ Results 4-12 are taken from Dickson, p. 298, 72, and p. 299, 73. 
For the right side is $E \cdot \epsilon_{2 t} \cdot I_{k} \cdot \epsilon_{t}$, while the left side is $I_{k} \cdot E \cdot \operatorname{conv}_{t}\left(E \cdot \epsilon_{2}\right)$ $=I_{k} \cdot E \cdot \epsilon_{t} \cdot \epsilon_{2 t}$.

$$
\sum_{m, n} \rho_{\nu, t}(m) \phi_{t k}(n)=N^{k} \rho_{\nu-k, t}(N)
$$

For $I_{\nu} \cdot \epsilon_{t} \cdot \operatorname{conv}_{t}\left(I_{t k} \cdot E^{-1}\right)=I_{\nu} \cdot \epsilon_{t} \cdot \epsilon_{t}^{-1} \cdot \operatorname{conv}_{t} I_{t k}=I_{\nu} \cdot\left(I_{k} \times \epsilon_{t}\right)=I_{k} \times\left(I_{\nu-k} \cdot \epsilon_{t}\right)$ $=I_{k} \times \rho_{\nu-k, t}$.

$$
\sum \sigma_{\nu-k}(m) \tau(n) m^{k}=\sum \rho_{k, t}(d) \rho_{\nu, t}(N / d) .
$$

For $\left(I_{k} \times \sigma_{\nu-k}\right) \cdot \operatorname{conv}_{t}\left(E^{2}\right)=I_{\nu} \cdot I_{k} \cdot \epsilon_{t} \cdot \epsilon_{t}=\rho_{\nu, t} \cdot \rho_{k, t}$.

$$
\sum \rho_{k, t}(m) \lambda(n)=\rho_{k, 2 t}(N) .
$$

For

$$
I_{k} \cdot \epsilon_{t} \cdot \operatorname{conv}_{t}(\lambda)=I_{k} \cdot \operatorname{conv}_{t}(E \cdot \lambda)=I_{k} \cdot \operatorname{conv}_{t} \epsilon_{2}=I_{k} \cdot \epsilon_{2 t} .
$$

In the following, $h$ is put for $\left(N / d_{2}\right)^{1 / 2}$.

$$
\sum \mu^{2}\left(d_{4}\right)=\sum \lambda(h) \text {. }
$$

For

$$
\epsilon_{4}=\operatorname{conv} \epsilon_{2}=\operatorname{conv}(E \cdot \lambda)=\epsilon_{2} \cdot \operatorname{conv} \lambda .
$$

Hence $\epsilon_{4} \cdot \lambda^{-1}=\lambda^{-1} \cdot \epsilon_{2} \cdot \operatorname{conv} \lambda=E \cdot \operatorname{conv} \lambda$.

$$
\sum E_{2}(h) \mu^{2}\left(d_{2}\right)=\sum \mu^{2}(h) .
$$

For

$$
\lambda^{-1} \cdot \operatorname{conv} E_{2}=\lambda^{-1} \cdot \operatorname{conv}\left(E \cdot \lambda^{-1}\right)=\lambda^{-1} \cdot \epsilon_{2} \cdot \operatorname{conv} \lambda^{-1}=E \cdot \operatorname{conv} \lambda^{-1} .
$$

$$
\sum \tau\left(h^{2}\right) \mu^{2}\left(d_{2}\right)=\sum E_{2}(h) .
$$

To prove this, note that the $k$ th convolute of $F(N)=f\left(N^{k}\right)$ is $f \times \epsilon_{k}$. Hence $\operatorname{conv}\left(E_{2} \cdot E\right)=\operatorname{conv} \tau_{2}=E^{2} \times \epsilon_{2}$.

Therefore

$$
\left(E^{2} \times \epsilon_{2}\right) \cdot \lambda^{-1}=\operatorname{conv}\left(E_{2} \cdot E\right) \cdot \lambda^{-1}=\operatorname{conv} E_{2} \cdot \epsilon_{2} \cdot \lambda^{-1}=E \cdot \operatorname{conv} E_{2} .
$$

$$
\sum \tau\left(d_{2}\right) \mu(h)=E_{2}(N) \text {. }
$$

For $E^{2} \cdot \operatorname{conv} E^{-1}=E^{2} \cdot \epsilon_{2}^{-1}=E_{2}$.

$$
\sum \mu^{2}(d) \phi_{k}\left(\frac{N}{d}\right)=\sum d_{2}{ }^{k} \mu(h) .
$$

For

$$
\lambda^{-1} \cdot \phi_{k}=\lambda^{-1} \cdot E^{-1} \cdot I_{k}=\operatorname{conv} E^{-1} \cdot I_{k} .
$$


(d) Zeta-series and simplex functions. When the necessary conditions of convergence are satisfied, we obtain by Dirichlet multiplication

Hence

$$
\begin{aligned}
\sum_{N=1}^{\infty} \frac{(f \cdot F)(N)}{N^{s}} & =\left\{\sum \frac{f(N)}{N^{s}}\right\}\left\{\frac{F(N)}{N^{s}}\right\}, \\
\sum_{N=1}^{\infty} \frac{f^{-1}(N)}{N^{s}} & =\left\{\sum \frac{f(N)}{N^{s}}\right\}^{-1} .
\end{aligned}
$$

$$
\begin{aligned}
& \sum E^{k}(N) / N^{s}=\{\zeta(s)\}^{k}, \\
& \sum \epsilon_{k}(N) / N^{*}=\zeta(k s), \\
& \sum I_{k}(N) / N^{*}=\zeta(s-k) .
\end{aligned}
$$

More generally, if $I_{r, t}$ is the $t$ th convolute of $I_{r}$, we have

$$
\sum I_{r, t}(N) / N^{s}=\zeta(t s-r) ; \sum I_{r, t}^{-1}(N) / N^{s}=\frac{1}{\zeta(t s-r)} .
$$

We shall refer to the functions $I_{r, t}, I_{r, t}^{-1}$ (where $r$ is unrestricted, and $t$ is a positive integer) as the elementary simplex functions. A composite of $\alpha$ functions of the type $I_{r, t}$ and $\beta$ functions of the type $I_{r, t}^{-1}$ will be called $a$ simplex function of structure $(\alpha, \beta)$.

THEOREM. The composite of simplex functions, and the inverse of a simplex function, are simplex. Also, every convolute of a simplex function $f$ is a simplex function with the same structure as $f$.

If $f$ is a simplex function, $\sum f(N) / N^{s}$, supposed convergent, can be evaluated as a product of quantities of the form $\zeta(t s+k)^{ \pm 1}$, where $k$ is arbitrary and $t$ is a positive integer; and conversely.

The first part follows from the definition. To prove the second part of the theorem, we observe that the $k$ th convolute of a simplex function must be the composite of the $k$ th convolutes of elementary simplex functions (Theorem VI). Since the $p$ th convolute of the $q$ th convolute of $f$ is its $p q$ th convolute, we have

$$
\operatorname{conv}_{k} I_{r, t}=I_{r, k t} ; \operatorname{conv}_{k} I_{r, t}^{-1}=I_{r, k t}^{-1} \text {. }
$$

Thus any convolute of a simplex function $f(N)$ is a composite of elementary simplex functions of the same type as the components of $f$, and so is a simplex function of the same structure as $f$.

The generating series of a simplex function $f$ to any base must clearly be of the form 


$$
f_{(p)}(x)=\frac{\left(1-p^{r_{1}} x^{s_{1}}\right)\left(1-p^{r_{2}} x^{s_{2}}\right) \cdots}{\left(1-p^{\rho_{1}} x^{\sigma_{1}}\right)\left(1-p^{\rho_{2}} x^{\sigma_{2}}\right) \cdots} .
$$

Since $I_{0}=E$, it follows that, among the elementary functions, the $I$ functions, as well as the functions $E$ and $E^{ \pm k}$, are simplex. The power units $\epsilon_{k}$, being convolutes of $E$, are simplex, while the power units $\pi_{k}$ are simplex because $\pi_{k} \cdot \epsilon_{k}=E$. Also, since $\lambda=\epsilon_{2} \cdot E^{-1}$, it follows that $\lambda$ is simplex; and lastly, $E_{2}$ is simplex, since $E_{2}=E \cdot \lambda^{-1}$. It is easy to verify that no others from among the elementary functions are simplex.

The values of the zeta-series determined by $\pi_{k}, E_{2}, \lambda$ are

$$
\begin{aligned}
\sum \pi_{k}(N) / N^{s} & =\zeta(s) / \zeta(k s), \\
\sum \lambda(N) / N^{s} & =\zeta(2 s) / \zeta(s), \\
\sum E_{2}(N) / N^{s} & =(\zeta(s))^{2} / \zeta(2 s)
\end{aligned}
$$

The product of simplex functions is not in general simplex. Ramanujan's result that

$$
\sum \frac{\sigma_{a}(N) \sigma_{b}(N)}{N^{s}}=\frac{\zeta(s) \zeta(s-a) \zeta(s-b) \zeta(s-a-b)}{\zeta(2 s-a-b)} *
$$

shows that the product of the simplex functions $\sigma_{a}, \sigma_{b}$ is also simplex. It does not appear to be easy to obtain any general theorem to cover such exceptional cases. The following results in this direction seem to be worthy of notice:

(1) The product of two elementary simplex functions is either an elementary simplex function, or a simplex function of structure $(1,1)$.

For

(a) $I_{r, t} \times I_{r^{\prime}, t^{\prime}}=I_{\rho, \tau}$, where $\tau$ is the least common multiple of $t, t^{\prime}$, and $\rho=\left(r \tau / t+r^{\prime} \tau / t^{\prime}\right)$.

(b) $I_{r, t} \times I_{r^{\prime}, t^{\prime}}^{-1}=I_{k r+r^{\prime}, t^{\prime}}^{-1}$ or $E_{0}$, according as $t^{\prime}=k t$, or is not a multiple of $t$.

(c) $I_{r, t}^{-1} \times I_{r^{\prime}, t^{\prime}}^{-1}=I_{2\left(r+r^{\prime}\right), 2 t} \cdot I_{r+r^{\prime}, t}^{-1}$ or $E_{0}$ according as $t$ is or is not equal to $t^{\prime}$.

(2) More generally, the product of two simplex functions of structure $(1,1)$ is also a simplex function of the same structure.

It follows from this, that $f \times \lambda$ is a simplex function when $f$ is simplex. For, $\lambda$ being linear, multiplication by it can be distributed to the simplex components of $f$ (Theorem VII); and since $\lambda$ is a simplex function of structure $(1,1)$, its product with an elementary simplex function must be simplex, by the present result.

(3) If $f$ is any simplex function of structure $(2,0)$ or $(1,1), f \times \epsilon_{2}$ is simplex, and therefore $f\left(N^{2}\right)$ is simplex.

* Collected Papers, p. 135, = Messenger of Mathematics, 1916, p. 83. 
For example $\sigma_{a}\left(N^{2}\right)$ and $\phi_{k}\left(N^{2}\right)$ are simplex functions of $N$.

(e) Crosses between elementary functions. Some of the most important functions of arithmetic are crosses between elementary functions. We mention a few such below.

(1) The function $T(N)=(-1)^{N-1}$ is easily verified to be a multiplicative function of $N$. For the base 2 it has the same element as $E_{-1}$, and for the remaining bases it has the same elements as $E$. We may conveniently represent it by the notátion

$$
T(N)=\left\{\begin{array}{ll}
2 & E_{-1} \\
* & E
\end{array}\right\}(N) .
$$

Hence, if $\phi$ is Euler's function,

$$
(\phi \cdot T)(N)=\left\{\begin{array}{ll}
2 & \phi \cdot E_{-1} \\
* & \phi \cdot E
\end{array}\right\}(N) .
$$

Now $\phi \cdot E=I$ and $\phi \cdot E_{-1}=I \cdot E^{-1} \cdot E_{-1}=I \cdot \lambda_{2}^{-1} ;$ but $\lambda_{2}^{-1}\left(2^{m}\right)=I^{-1}\left(2^{m}\right)$, and so $\left(\phi \cdot E_{-1}\right)\left(2^{m}\right)=E_{0}\left(2^{m}\right)$. Hence, we have the result of Liouville*

$$
\sum(-1)^{d-1} \phi\left(\frac{N}{d}\right)=0 \text { or } N \text {, according as } N \text { is even or odd. }
$$

(2) Let $R(N)$ denote the number of representations of $N$ as the sum of two squares, all representations being counted twice, with the exception of those of the form $0^{2}+M^{2}, M^{2}+M^{2}$, which are counted only once. It is known that $R(N)$ is equal to the excess of the number of divisors of $N$, of the form $4 k+1$, over the number of those of the form $4 k-1$. Thus $R(N)=(E \cdot$ \& $)(N)$, where $\&(N)$ is the cross between $E, \lambda, E_{0}$, given by

$$
\xi=\left\{\begin{array}{cc}
2 & E_{0} \\
4 k+1 & E \\
4 k-1 & \lambda
\end{array}\right\} .
$$

6. Compounding of functions of a single argument. We confine ourselves in this subsection to functions of a single argument; the compounding of functions of several arguments is treated in Section V.

By a block-factor $\delta$ of $M$ we shall mean a factor $\delta$ which is relatively prime to $M / \delta$. If $\delta$ is a block-factor, the complementary factor $M / \delta$ is also a blockfactor. We have to consider 1 and $M$ also as complementary block-factors of $M$. If $f(M), F(M)$ are given multiplicative functions, the sum

$$
\sum f(\delta) F(M / \delta)
$$

* Dickson, p. 121, 29. 
extended to all the block-factors $\delta$ of $M$, is easily seen to be a multiplicative function $\Phi$ of $M$. We call $\Phi$ the compound of $f$ and $F$, and denote it by the symbol $f \oplus F$.

If the generating series of $f, F$ are

$$
\begin{aligned}
& f_{(p)}^{\prime}(x)=1+a_{1} x+a_{2} x^{2}+\cdots, \\
& F_{(p)}(x)=1+b_{1} x+b_{2} x^{2}+\cdots,
\end{aligned}
$$

it follows immediately that the generating series of their compound is given by

$$
(f \oplus F)_{(p)}(x)=1+\left(a_{1}+b_{1}\right) x+\left(a_{2}+b_{2}\right) x^{2}+\cdots .
$$

The constant term here is not 2 , but 1 , as must be the case with every multiplicative function. Similarly the generating series of the compound of $r$ functions is obtained by adding the corresponding generating series of the functions, and replacing the constant term $r$ by 1 , in the sum. If we say that two power series in the same variables are equivalent (symbol $\sim$ ), when they differ only in their constant terms, we have

Theorem XII. The generating series of a compound is equivalent to the sum of the generating series, to the same base, of the functions compounded.

Hence compounding is associative and commutative.

Theorem XIII. The compounding operation distributes multiplication.

For, let the functions $f_{1}, f_{2}, f$ have the generating series

$$
\begin{aligned}
& f_{1(p)}(x)=1+a_{1} x+a_{2} x^{2}+\cdots, \\
& f_{2(p)}(x)=1+b_{1} x+b_{2} x^{2}+\cdots, \\
& f_{(p)}(x)=1+c_{1} x+c_{2} x^{2}+\cdots .
\end{aligned}
$$

Then

$$
\left\{f \times\left(f_{1} \oplus f_{2}\right)\right\}_{(p)}(x)=1+c_{1}\left(a_{1}+b_{1}\right) x+c_{2}\left(a_{2}+b_{2}\right) x^{2}+\cdots,
$$

while

$$
\left\{\left(f \times f_{1}\right) \oplus\left(f \times f_{2}\right)\right\}_{(p)}(x)=1+\left(c_{1} a_{1}+c_{1} b_{1}\right) x+\left(c_{2} a_{2}+c_{2} b_{2}\right) x^{2}+\cdots,
$$

which establishes the distributive property.

THEOREM XIV. Compounding is quasidistributive with respect to composition.

For, consider the composite of $f$ with the compound of $f_{1}, f_{2}, \cdots, f_{r}$. We have 


$$
\begin{aligned}
\left\{f \cdot\left(f_{1} \oplus f_{2} \oplus \cdots \oplus f_{r}\right)\right\}_{(p)}(x) & \\
=f_{(p)}(x) \times\left\{f_{1(p)}(x)+f_{2(p)}(x)\right. & \left.+\cdots+f_{r(p)}(x)+1-r\right\} \quad\{\text { Theorem XII }\} \\
& =\sum_{i} f_{(p)}(x) f_{i(p)}(x)+(1-r) f_{(p)}(x) \\
& =\sum_{i}\left(f \cdot f_{i}\right)_{(p)}(x)+\left(E_{1-r} \times f\right)_{(p)}(x)-r \\
& \sim \sum_{i}\left(f \cdot f_{i}\right)_{(p)}(x)+\left(E_{1-r} \times f\right)_{(p)}(x) .
\end{aligned}
$$

Hence

$$
f \cdot\left(f_{1} \oplus f_{2} \oplus \cdots \oplus f_{r}\right)=\sum_{i}\left(f \cdot f_{i}\right) \oplus\left(E_{1-r} \times f\right) .
$$

This formula is fundamental. The term "quasidistributive" in the enunciation refers to the occurrence of the additional term $\oplus\left(E_{1-r} \times f\right)$, which marks the failure of the distributive property.

The compound of two linear functions is capable of simple expression as a composite.

THEOREM XV. The compound of two linear functions $L_{1}, L_{2}$ is the composite of $L_{1}, L_{2}$ and the inverse of the second convolute (or root-composite) of $L_{12}=\left(L_{1} \times L_{2}\right)$.

More generally, the compound of the rth convolute of $L_{1}$ and the sth convolute of $L_{2}$, is their continued composite with the $(r+s)$ th convolute of $L_{12}^{-1}$.

For let

$$
L_{1(p)}(x)=\frac{1}{1-\alpha x}, L_{2(p)}(x)=\frac{1}{1-\beta x}
$$

so that

$$
\left(\operatorname{conv}_{r} L_{12}\right)_{(p)}(x)=\frac{1}{1-\alpha \beta x^{r}}
$$

Then

$$
\begin{aligned}
\left(L_{1} \oplus L_{2}\right)_{(p)}(x) & =\frac{1}{1-\alpha x}+\frac{1}{1-\beta x}-1=\frac{1-\alpha \beta x^{2}}{(1-\alpha x)(1-\beta x)} \\
& =\left(L_{1} \cdot L_{2} \cdot \operatorname{conv} L_{12}^{-1}\right)_{(p)}(x) ; \\
\left(\operatorname{conv}_{r} L_{1} \oplus \operatorname{conv}_{s} L_{2}\right)_{(p)}(x) & =\frac{1}{1-\alpha x^{r}}+\frac{1}{1-\beta x^{8}}-1=\frac{1-\alpha \beta x^{r+s}}{\left(1-\alpha x^{r}\right)\left(1-\beta x^{8}\right)} \\
& =\left(\operatorname{conv}_{s} L_{1} \cdot \operatorname{conv}_{s} L_{2} \cdot \operatorname{conv}_{r+s} L_{12}^{-1}\right)_{(p)}(x) .
\end{aligned}
$$

As a corollary from this theorem it follows that the compound of simplex linear functions is also simplex. For instance, if 


$$
\begin{aligned}
S_{a, b} & =I_{a} \oplus I_{b}=I_{a} \cdot I_{b} \cdot I_{a+b, 2}^{-1}, \\
S_{a}{ }^{\prime} & =I_{a} \oplus \lambda=I_{a} \cdot \lambda \cdot \operatorname{conv}\left(I_{a} \times \lambda\right)^{-1}=I_{a} \cdot \lambda \cdot I_{a, 2} \cdot I_{2 a, 4}^{-1},
\end{aligned}
$$

then $S_{a, b}, S_{a}^{\prime}$ are simplex functions, and

$$
\begin{aligned}
& \sum_{N} \frac{S_{a, b}(N)}{N^{t}}=\frac{\zeta(t-a) \zeta(t-b)}{\zeta(2 t-a-b)} \\
& \sum_{N} \frac{S_{a}{ }^{\prime}(N)}{N^{t}}=\frac{\zeta(2 t) \zeta(t-a) \zeta(2 t-a)}{\zeta(t) \zeta(4 t-2 a)} .
\end{aligned}
$$

We note also that $E_{2}$ is a particular case of $S_{a, b}$, namely, $S_{0,0}$.

The conjugate of $f(M)$ is the function conj $f \operatorname{defined~by~}$

$$
f \oplus \operatorname{conj} f=E_{0} .
$$

If the generating series of $f$ to any base is $1+\sum_{m=1} a_{m} x^{m}$, the generating series of conj $f$ to the same base is evidently $1-\sum_{m=1} a_{m} x^{m}$. Hence an alternative definition of conj $f$ is

$$
\operatorname{conj} f=f \times E_{-1} .
$$

The conjugate function has evidently the properties

$$
\operatorname{conj} \operatorname{conj} f=f ; \operatorname{conj}(f \times \phi)=\operatorname{conj} f \times \phi=f \times \operatorname{conj} \phi ;
$$

conjugate of a compound = compound of the conjugates.

The $E$-functions play an important part in relation to the compounding operation. In fact, their algebra under compounding and functional multiplication is isomorphic with the algebra of ordinary numbers under addition and multiplication, so that any identity between ordinary numbers can be translated into a relation between $E$-functions. Thus

$$
\begin{gathered}
E_{r} \oplus E_{s}=E_{r+s} ; E_{r} \times E_{s}=E_{r s} ; \operatorname{conj} E_{r}=E_{-r} ; \\
f \oplus f \oplus \cdots(\text { to } r \text { terms })=f \times E_{r} .
\end{gathered}
$$

We give below several results involving the $E$-functions.

Example 5. $f \cdot\left(E_{r} \times \phi\right)=f \cdot(\phi \oplus \phi \oplus \cdots)=\left\{(f \cdot \phi) \times E_{r}\right\} \oplus\left(E_{1-r} \times f\right)$ (Theorem XIV). As particular cases, we have

$$
\begin{array}{rlrl}
\operatorname{conj}(f \cdot \phi) & =\{f \cdot \operatorname{conj} \phi\} \oplus\left(E_{-2} \times f\right) & & \{r=-1\} ; \\
f \cdot E_{r} & =\left\{(f \cdot E) \times E_{r}\right\} \oplus E_{1-r} \times f & \{\phi=E\} ; \\
f \cdot\left(E_{r} \times f^{-1}\right) & =E_{1-r} \times f & & \left\{\phi=f^{-1}\right\} ; \\
f \cdot \operatorname{conj} f^{-1} & =E_{2} \times f & & \left\{\phi=f^{-1} ; r=-1\right\} .
\end{array}
$$


Example 6.

$$
\begin{aligned}
\left(E_{r} \times f\right) \cdot\left(E_{s} \times f^{-1}\right) & =\{f \oplus f \oplus \cdots\} \cdot\left\{f^{-1} \oplus f^{-1} \oplus \cdots\right\} \\
& =\left\{E_{r(1-s)} \times f\right\} \oplus\left\{E_{s(1-r)} \times f^{-1}\right\} .
\end{aligned}
$$

Putting $r=s=-1$, we have conj $f \cdot \operatorname{conj} f^{-1}=E_{-2} \times\left(f \oplus f^{-1}\right)$.

Example 7.

$$
E^{r} \cdot E_{s}=\left(E^{r+1} \times E_{s}\right) \oplus\left(E^{r} \times E_{1-s}\right) .
$$

In particular, putting $r=1, E^{2} \times E_{s}=\left(E_{s} \cdot E\right) \oplus E_{s-1}$.

$$
E_{r}^{-1} \times E_{r-1}=\lambda_{1-r} \times E_{r} .
$$

For, since $E_{r} \cdot \lambda_{1-r}=E($ II $\S 5(\mathrm{~b}))$,

$$
\begin{aligned}
\lambda_{1-r} \times E_{r} & =\left(E_{r}^{-1} \cdot E\right) \times E_{r} \\
& =\left(E_{r}^{-1} \cdot E_{r}\right) \oplus\left(E_{r-1} \times E_{r}^{-1}\right)\left(\text { Example 5) }=E_{r}^{-1} \times E_{r-1} .\right.
\end{aligned}
$$

In particular, $E_{2}^{-1}=E_{2} \times \lambda$, as has been already proved.

\section{Example 8.}

$$
\begin{aligned}
& E_{r} \cdot E_{s}=\tau_{r s} \oplus E_{(1-r)(s-1)} ; \\
& \tau_{2 r} \cdot E=\tau \times \tau_{r} .
\end{aligned}
$$

For

$$
\begin{aligned}
E_{r} \cdot E_{s} & =\left\{\left(E_{r} \cdot E\right) \times E_{s}\right\} \oplus\left(E_{1-s} \times E_{r}\right) \\
& =\left(E^{2} \times E_{r} \times E_{s}\right) \oplus\left(E_{1-r} \times E_{s}\right) \oplus\left(E_{1-s} \times E_{r}\right) \\
& =\left(E^{2} \times E_{r s}\right) \oplus E_{r+s-2 r s} \\
& =\left(E_{r s} \cdot E\right) \oplus E_{r s-1} \oplus E_{r+s-2 r s} \text { (Example 5,(2)) } \\
& =\tau_{r s} \oplus E_{(1-r)(s-1)} ;
\end{aligned}
$$

and

$$
\begin{aligned}
\tau \times \tau_{r}=E^{2} \times\left(E \cdot E_{r}\right) & =E^{2} \times\left\{\left(E^{2} \times E_{r}\right) \oplus E_{1-r}\right\} \text { (Example 5) } \\
& =\left(E^{2} \times E^{2} \times E_{r}\right) \oplus\left(E^{2} \times E_{1-r}\right) \\
& =\left\{\left(E^{2} \cdot E_{2}\right) \times E_{r}\right\} \oplus\left(E^{2} \times E_{1-r}\right) \text { (Example 3) } \\
& =E^{2} \cdot\left(E_{2} \oplus E_{2} \oplus \cdots \text { to } r\right. \text { terms) (Theorem XIV) } \\
& =E^{2} \cdot E_{2 r}=\tau_{2 r} \cdot E .^{*}
\end{aligned}
$$

* The results in Examples 5-8 are proved here only for the case in which $r, s$ are positive integers; they may be easily proved for arbitrary values of $r, s$, by considering the generating series. It is also possible to deduce their truth for arbitrary values of $r, s$, from their truth when $r, s$ are integers, by purely logical considerations. 


\section{Example 9.}

(a) $\sum f_{1}\left(d_{1}\right) f_{2}\left(d_{2}\right) \cdots f_{k}\left(d_{k}\right)$ summed for all sets of divisors $d_{1}, d_{2}, \cdots, d_{k}$ of $N$, such that every two are relatively prime, is equal to $\left\{\left(f_{1} \oplus f_{2} \oplus \cdots\right.\right.$ $\left.\left.\oplus f_{k}\right) \cdot E\right\}(N)$.

(b) $\sum f_{1}\left(d_{1}\right) f_{2}\left(d_{2}\right) \cdots f_{k}\left(d_{k}\right)$ summed for all sets of divisors $d_{1}, d_{2}, \cdots, d_{k}$ of $N$, with the least common multiple $N$, is equal to $\left[\left\{\left(f_{1} \cdot E\right) \times\left(f_{2} \cdot E\right) \times \cdots\right.\right.$ $\left.\left.\times\left(f_{k} \cdot E\right)\right\} \cdot E^{-1}\right](N)$.

The first part is obvious. To prove (b), we observe that if the required sum be $F(N)$, then $(F \cdot E)(N)$ is equal to $\sum f_{1}\left(d_{1}\right) f_{2}\left(d_{2}\right) \cdots f_{k}\left(d_{k}\right)$ summed for all sets of divisors $d_{1}, d_{2}, \cdots, d_{k}$ of $N$; that is, to $\left\{\left(f_{1} \cdot E\right) \times\left(f_{2} \cdot E\right) \cdots\right.$ $\left.\times\left(f_{k} \cdot E\right)\right\}(N)$.

Putting $k=2$, we can deduce an important result from these two theorems. Let $d_{1}, d_{2}$ be divisors having the least common multiple $N$. If their greatest common divisor is $\delta$, we can write $d_{1}=\delta t_{1} ; d_{2}=\delta t_{2}$, where $t_{1}, t_{2}$ are relatively prime, and $t_{1} t_{2}=N / \delta$. Hence, if $L_{1}, L_{2}$ are linear functions.

$$
\begin{aligned}
\sum L_{1}\left(d_{1}\right) L_{2}\left(d_{2}\right) & =\sum L_{1}(\delta) L_{2}(\delta) L_{1}\left(t_{1}\right) L_{2}\left(t_{2}\right) \\
& =\sum L_{12}(\delta) L_{1}\left(t_{1}\right) L_{2}\left(t_{2}\right), \text { where } L_{12}=L_{1} \times L_{2}, \\
& =\left\{L_{12} \cdot\left(L_{1} \oplus L_{2}\right)\right\}(N)=\left\{L_{12} \cdot L_{1} \cdot L_{2} \cdot \operatorname{conv} L_{12}^{-1}\right\}(N)
\end{aligned}
$$

(Theorem XV).

But the left side is, by the present theorem, equal to $\left\{\left(L_{1} \cdot E\right) \times\left(L_{2} \cdot E\right)\right\} \cdot E^{-1}$. Hence, if $L_{1}, L_{2}$ be linear functions,

$$
\left(L_{1} \cdot E\right) \times\left(L_{2} \cdot E\right)=L_{12} \cdot L_{1} \cdot L_{2} \cdot E \cdot \operatorname{conv} L_{12}^{-1} .
$$

In particular, $\left(I_{a} \cdot E\right) \times\left(I_{b} \cdot E\right)=I_{a+b} \cdot I_{a} \cdot I_{b} \cdot E \cdot I_{a+b, 2}^{-1}$, which proves Ramanujan's result (II $\$ 5(d))$ that

$$
\sum_{N} \frac{\sigma_{a}(N) \sigma_{b}(N)}{N^{s}}=\frac{\zeta(s) \zeta(s-a) \zeta(s-b) \zeta(s-a-b)}{\zeta(2 s-a-b)} .
$$

\section{Section III. Rational functions of ONE ARgument}

1. The concept of rational function. A function of $N$ which is the composite of $r$ linear functions will be called a rational integral function of degree $r^{*}$. If $f(N)$ be such a function,

$$
\begin{aligned}
& f_{(p)}(x)=\frac{1}{\left(1-a_{1} x\right)\left(1-a_{2} x\right) \cdots\left(1-a_{r} x\right)} ; \\
& f_{(p)}^{-1}(x)=\left(1-a_{1} x\right)\left(1-a_{2} x\right) \cdots\left(1-a_{r} x\right),
\end{aligned}
$$

* The notion, though not the actual expression "rational integral function," as well as Theorem $\mathrm{XVI}$, occur in the last part of my note $O$ n the inversion of multiplicative arithmetic functions, Journal of the Indian Mathematical Society, October, 1927. 
so that $f^{-1}\left(p^{n}\right)=0$, if $n>r$. Hence

THEOREM XVI. The condition that $f(N)$ be a rational integral function of degree $r$ is the vanishing of $f^{-1}(N)$ for all values of $N$ divisible by an $(r+1)$ th power.

The composite of a rational integral function $P_{r}$ of degree $r$, and the inverse of a rational integral $Q_{s}$ of degree $s$, will be called a rational function of degree $(r, s) ; P_{r}$ will be called the integral component, and $Q_{s}^{-1}$, the inverse component, of the rational function. The linear components of $P_{r}$ and $Q_{s}$ will also be called, respectively, the linear and the inverse-linear components of the rational function $P_{r} \cdot Q_{s}^{-1}$. Also, a rational function will be said to be expressed in rational form, when its integral and inverse components are put in explicit evidence.

It follows from the above that the generating series $f_{(p)}(x)$ of a rational function $f$ of degree $(r, s)$ is the expansion of a rational function of $x$, whose denominator and numerator are of degrees $r, s$ respectively; $f_{(p)}(x)$ is thus a recurring series of order $r$ according to the usual definition.

Rational functions of degree $(1,1)$ will be called totients.

The fundamental theorem in the theory of rational functions may be stated as follows:

Theorem XVII. All the processes of our calculus, excepting division, ${ }^{*}$ are rational processes, in the sense that, when performed on rational functions, they yield only rational functions.

The truth of this theorem is obvious from the definition, so far as composition and inversion are concerned. For, the inverse of the rational function $P_{r} \cdot Q_{s}^{-1}$ of degree $(r, s)$ is the rational function $Q_{s} \cdot P_{r}^{-1}$ of degree $(s, r)$; and the composite of the rational functions $\left(P_{r} \cdot Q_{s}^{-1}\right),\left(P_{\rho} \cdot Q_{\sigma}^{-1}\right)$ is the rational function $\left(P_{r} \cdot P_{\rho}\right) \cdot\left(Q_{s} \cdot Q_{\sigma}\right)^{-1}$, which is in general of degree $(r+\rho, s+\sigma)$. It remains, therefore, to prove the fundamental theorem for multiplication and compounding.

We shall prove in a general manner that the product of two rational functions is a rational function. Let $f, \phi$ be two rational functions, whose integral components are of degree $r, \rho$, respectively. Then the generating series

$$
\begin{aligned}
& f_{(p)}(x)=1+a_{1} x+a_{2} x^{2}+\cdots, \\
& \phi_{(p)}(x)=1+\alpha_{1} x+\alpha_{2} x^{2}+\cdots
\end{aligned}
$$

* That division is not a rational process may be seen from an example. The series $\sum n x^{n}$ is a recurring series, but the series $\sum x^{n} / n$ is not a recurring series. Thus the quotient of two rational functions need not be rational. (Cf. the form of the generating series of a quotient obtained in II \$1.) 
are recurring series of orders $r, \rho$, respectively. To prove that $(f \times \phi)$ is a rational function, we have to show that the series

$$
(f \times \phi)_{(p)}(x)=1+a_{1} \alpha_{1} x+a_{2} \alpha_{2} x^{2}+\cdots
$$

is also a recurring series (in fact, of order $r \rho$, as we shall see). To prove this, observe that when $m$ is sufficiently large, the two sets of quantities

$$
\begin{gathered}
a_{m}, a_{m-1}, \cdots, a_{m-t}, \\
\alpha_{m}, \alpha_{m-1}, \cdots, \alpha_{m-t}
\end{gathered}
$$

are connected respectively by $t-r+1$ and $t-\rho+1$ linear equations. On multiplying each of the linear equations between the $a$ 's by each $\alpha$, and each of the equations between the $\alpha$ 's by each $a$, we obtain $(t+1)(2 t-r-\rho+2)$ linear equations connecting the $(t+1)^{2}$ quantities $a_{\mu} \alpha_{\nu}$. Of these, $(t-r+1)$ $\cdot(t-\rho+1)$ equations are redundant, since we obtain this number on multiplication of each of the $t-r+1$ equations between the $a$ 's with each of the $t-\rho+1$ equations between the $\alpha$ 's. The remaining $(t+1)^{2}-r \rho$ equations between the products $a_{\mu} \alpha_{\nu}$ are in general linearly independent; if the $(t+1)^{2}$ $-(t+1)$ products $a_{\mu} \alpha_{\nu}(\mu \neq \nu)$ are to be just capable of elimination from these, we must have

$$
(t+1)^{2}-r \rho=(t+1)^{2}-(t+1)+1
$$

or

$$
t=r \rho .
$$

Hence the series $1+a_{1} \alpha_{1} x+a_{2} \alpha_{2} x^{2}+\cdots$ is a recurring series of order $r \rho$, as was to be proved.

This result may be looked upon as a particular case of Hadamard's theorem on the multiplication of singularities, ${ }^{*}$ according to which, the singularities of the analytic function represented by the power series $\sum a_{r} b_{r} x^{r}$ are included among the products $\alpha_{\lambda} \beta_{\mu}(\lambda, \mu=1,2, \ldots)$, the $\alpha$ 's and $\beta$ 's being the singularities of the analytic functions represented by the respective series $\sum a_{r} x^{r}$ and $\sum b_{r} x^{r}$; for, if $\sum a_{r} x^{r}$ and $\sum b_{r} x^{r}$ are both rational functions, then the $\alpha$ 's and $\beta$ 's, as well as their products, must be finite in number, so that $\sum a_{r} b_{r} x^{r}$ has only a finite number of isolated singularities and is therefore a rational function. This theorem shows also that the integral components of the product-function $f \times \phi$ may be specified in terms of the integral components of $f$ and $\phi$. We shall indicate below a second proof of this theorem, which has the advantage of specifying the integral component of the productfunction.

* Bieberbach, Encyklopädie, Band II, Teil 3, erste Hälfte, p. 464. 
The behavior of inverses of integral functions under multiplication, composition and compounding is noteworthy; we have

THEOREM XVIII. The product, composite, or compound of inverses of integral functions is also the inverse of an integral function.

To prove this, we have only to observe that the generating series of inverses of integral functions to the base $p$, are all finite polynomials $1+\sum a_{m} x^{m}$, $1+\sum b_{m} x^{m}$, etc. Hence each of the power series $1+\sum a_{m} b_{m} x^{m},\left(1+\sum a_{m} x^{m}\right)$ $\cdot\left(1+\sum b_{m} x^{m}\right)$ and $1+\sum\left(a_{m}+b_{m}\right) x^{m}$ is a finite polynomial.

We may also note that the composite of two rational integral functions is a rational integral function. Thus, composition is not only a rational, but also an integral, process. It will appear that it is the only one of our processes which is integral.

2. Properties of totients. Totients being rational functions of degree $(1,1)$, are functions of the form $L_{1} \cdot L_{2}^{-1}$, where $L_{1}, L_{2}$ are linear. Two special types of totients are of great importance. The totients of the form $L_{1} \cdot E^{-1}$ (including Euler's and Jordan's functions as special cases) will be called enumerative totients. It follows from this definition, that the integral of an enumerative totient is a linear function.

The inverse of an enumerative totient $T=L_{1} \cdot E^{-1}$ is of the form $T^{-1}=E \cdot L_{1}^{-1}$, and will be called a level totient. The property of the level totient which gives it its name is $T^{-1}(p)=T^{-1}\left(p^{2}\right)=\cdots=T^{-1}\left(p^{n}\right)$, where $p$ is any prime. The level totient is consequently the integral (=composite with $E$, II $\S 3$ ) of the inverse of a linear function. Since $E_{k}(p)=E_{k}\left(p^{2}\right)=\cdots$ it follows that the elementary functions $E_{k}$ are level totients, though of a special type, since the value of $E_{k}(p)$ is independent of the prime $p$. Hence, also, the general level totient may be regarded as a cross between (in general, an infinite number of) $E$-functions. Consequently, from the independence of the elements of a multiplicative function, we can deduce properties of level totients from known theorems on $E$-functions. For example

Example 10. The product and compound of level totients are level totients.

To deduce the truth of this for level totients, from its truth for $E$-functions, we observe that

Hence

$$
\begin{gathered}
E_{k}=\lambda_{1-k}^{-1} \cdot E ; E_{k} \times E_{k^{\prime}}=E_{k k^{\prime}}=\lambda_{1-k k^{\prime}}^{-1} \cdot E \\
\lambda_{1-k k^{\prime}}^{-1}=\lambda_{1-k}^{-1} \oplus \lambda_{1-k^{\prime}}^{-1} \oplus\left(\lambda_{1-k}^{-1} \times \lambda_{1-k^{\prime}}^{-1}\right)
\end{gathered}
$$

$$
\left(\lambda_{1-k}^{-1} \cdot E\right) \times\left(\lambda_{1-k^{\prime}}^{-1} \cdot E\right)=\left\{\lambda_{1-k}^{-1} \oplus \lambda_{1-k^{\prime}}^{-1} \oplus\left(\lambda_{1-k}^{-1} \times \lambda_{1-k^{\prime}}^{-1}\right)\right\} \cdot E .
$$


Now, if $T_{1}=L_{1}^{-1} \cdot E, T_{2}=L_{2}^{-1} \cdot E$ are two given level totients, and if $T_{1}, T_{2}$ have the same elements as $E_{k}, E_{k^{\prime}}$, for the base $p$, then for the same base, $L_{1}, L_{2}$ should have the same elements as $\lambda_{1-k}, \lambda_{1-k^{\prime}}$. Hence the two functions

$$
\begin{gathered}
\left(L_{1}^{-1} \cdot E\right) \times\left(L_{2}^{-1} \cdot E\right), \\
\left\{L_{1}^{-1} \oplus L_{2}^{-1} \oplus\left(L_{1}^{-1} \times L_{2}^{-1}\right)\right\} \cdot E
\end{gathered}
$$

have identical elements for every base, and are therefore identical. Since the part within braces is the inverse of a linear function (Theorem XVIII), this expresses the product of the level totients in rational form as a level totient.

In the same way, since

$$
E_{r} \oplus E_{s}=E_{r+s}=\lambda_{1-r-s}^{-1} \cdot E=\left(\lambda_{1-r}^{-1} \oplus \lambda_{1-s}^{-1} \oplus \lambda^{-1}\right) \cdot E
$$

it follows that the compound of the level totients $L_{1}^{-1} \cdot E, L_{2}^{-1} \cdot E$ is the level totient given by

$$
\left(L_{1}^{-1} \oplus L_{2}^{-1} \oplus \lambda^{-1}\right) \cdot E .
$$

Example 11. It was proved that

$$
(f \cdot \phi) \times E_{r}=f \cdot\left(\phi \times E_{r}\right) \oplus\left(E_{r-1} \times f\right) \text { (Example 5). }
$$

Hence, if $T$ is a level totient,

$$
(f \cdot \phi) \times T=f \cdot(\phi \times T) \oplus\left\{f \times\left(T \oplus E_{-1}\right)\right\} .
$$

It follows from this, that if $f$ is a rational function, $f \times T$ is also a rational function, with the same integral component as $f$. For, if $f=P \cdot Q^{-1}$, where $P, Q$ are rational integral functions, then

$$
\begin{aligned}
P^{-1} \cdot(f \times T) & =\left\{\left(P^{-1} \cdot f\right) \times T\right\} \oplus\left\{P^{-1} \times(\operatorname{conj} T \oplus E)\right\} \\
& =\left\{Q^{-1} \times T\right\} \oplus\left\{P^{-1} \times(\operatorname{conj} T \oplus E)\right\} .
\end{aligned}
$$

Since the expression on the right is the inverse of an integral function, this expresses $f \times T$ in rational form.

The enumerative totients do not form, like the level totients, a closed system under multiplication, or compounding, but they possess this property for a different process of combination. It was proved that the function $f(N)$ defined by

$$
f(N)=\sum f_{1}\left(d_{1}\right) f_{2}\left(d_{2}\right) \cdots f_{k}\left(d_{k}\right),
$$

where the summation is for all sets of divisors $d_{1} d_{2} \cdots d_{k}$ having the least common multiple $N$, is equal to $\left[\left\{\left(f_{1} \cdot E\right) \times\left(f_{2} \cdot E\right) \times \cdots \times\left(f_{k} \cdot E\right)\right\} \cdot E^{-1}\right](N)$ (Example 9). The function $f$ may be called the combinant of $f_{1}, f_{2}, \cdots, f_{k}$. From the expression for the combinant, we immediately have 
THEOREM XIX. The combinant $f$ of any number of enumerative totients $f_{1}, f_{2}, \cdots, f_{k}$ is also an enumerative totient; the linear component of $f$ is the product of the linear components of $f_{1}, f_{2}, \cdots, f_{k}$.

As an example, the Jordan function $\phi_{k}$ is the combinant of $k$ functions each identical with the Euler totient $\phi{ }^{*}$

The general totient may be investigated by means of a canonical form, which expresses it as the product of a level totient and a linear function.

THEOREM XX. The totient $L_{1} \cdot L_{2}^{-1}=T$ is the product of its integral component $L_{1}$ by the level totient $L \cdot E^{-1}$, where $L$ is the linear function defined by $L(N)=L_{2}(N) / L_{1}(N)$.

For, let

$$
L_{1(p)}(x)=\frac{1}{1-\alpha x}, \quad L_{2(p)}(x)=\frac{1}{1-\beta x} ;
$$

then

$$
T_{(p)}(x)=\frac{1-\beta x}{1-\alpha x}=1+\frac{\alpha-\beta}{\alpha} \cdot \alpha x+\frac{\alpha-\beta}{\alpha} \cdot \alpha^{2} x^{2}+\cdots=\left(L_{1} \times K\right)_{(p)}(x)
$$

where

$$
K_{(p)}(x)=1+\frac{\alpha-\beta}{\alpha}\left(1+x+x^{2}+\cdots\right)=\frac{\cdot 1-\frac{\beta}{\alpha} x}{1-x}=\left(L^{-1} \cdot E\right)_{(p)}(x),
$$

where

$$
L_{(p)}(x)=\frac{1}{1-\frac{\beta}{\alpha} x} ; \text { or } L(N)=\frac{L_{2}(N)}{L_{1}(N)} .
$$

The theorem will however become invalid if $L_{1}$ vanishes for finite values of its argument, that is, if $L_{1}$ has common elements with $E_{0}$.

We may call the totient $K=L \cdot E^{-1}$ the associated level totient of $T$. We also observe that the general totient can be obtained by multiplication of the general level totient and the general linear function.

We shall use this expression for the totient to prove

TheOREM XXI. The product of the totients $T_{1}, T_{2}$ is a totient $T$, whose linear component is the product of the linear components of $T_{1}, T_{2}$.

For, let $K_{1}, K_{2}$ be the associated level totients of $T_{1}, T_{2}$, and let

\footnotetext{
* This property of the Jordan function is due to von Sterneck; see Dickson, p. 151, 215.
} 


$$
T_{1}=L_{1} \cdot P_{1}^{-1} ; T_{2}=L_{2} \cdot P_{2}^{-1}
$$

Then

$$
T=T_{1} \times T_{2}=L_{1} \times L_{2} \times K_{1} \times K_{2}=L \times K,
$$

where $L=L_{1} \times L_{2}$, and $K$ is the level totient which is the product of the level totients $K_{1}, K_{2}$. Thus the product is a totient whose linear component is $L_{1} \times L_{2}=L$. The inverse-linear component of $T$ may be easily shown to be

$$
\left(L_{1} \times P_{2}^{-1}\right) \oplus\left(L_{2} \times P_{1}^{-1}\right) \oplus\left(P_{1}^{-1} \times P_{2}^{-1}\right) .
$$

This proof will, however, become invalid, if $L_{1}$ or $L_{2}$ has the same element as $E_{0}$ for certain prime bases $p$. For such prime bases, however, either $T_{1}$ or $T_{2}$ or both must reduce to the inverse of a linear function, and therefore, also, their product. Since the inverse of a linear function may be considered as a degenerate case of the totient, the theorem is always true.

As a special case of the theorem, we may note that the conjugate $T^{\prime}$ of a totient $T$, being equal to the product $T \times E_{-1}$, is also a totient. By Example 11 , the linear components of $T, T^{\prime}$ are the same.

3. Compound of rational functions. Let $F=A: B^{-1}, \Phi=C \cdot D^{-1} ; A, B$, $C, D$ being rational integral functions. Also let $L$ be the least common composite of $A, C$, that is, the integral function of least degree, which is of each of the forms $A \cdot L_{1}, C \cdot L_{2}$, where $L_{1}, L_{2}$ are rational and integral. Then

$$
\begin{aligned}
F \oplus \Phi & =\left(A \cdot B^{-1}\right) \oplus\left(C \cdot D^{-1}\right) \\
& =\left\{\left(A \cdot L_{1}\right) \cdot\left(B \cdot L_{1}\right)^{-1}\right\} \oplus\left\{\left(C \cdot L_{2}\right) \cdot\left(D \cdot L_{2}\right)^{-1}\right\} \\
& =L \cdot\left\{D \cdot\left(B \cdot L_{1}\right)^{-1} \oplus\left(D \cdot L_{2}\right)^{-1}\right\} \oplus L \\
& =L \cdot\left\{\left(B \cdot L_{1}\right)^{-1} \oplus\left(D \cdot L_{2}\right)^{-1}\right\} \oplus\left(E_{2} \times L\right) \oplus\left(E_{-1} \times L\right) .
\end{aligned}
$$

Now

$$
E_{2} \times L=L \cdot \operatorname{conj} L^{-1}(\text { Example 5(4)) }
$$

Hence

$$
F \oplus \Phi=L \cdot\left\{\left(B \cdot L_{1}\right)^{-1} \oplus\left(D \cdot L_{2}\right)^{-1} \oplus \operatorname{conj} L^{-1}\right\} .
$$

This shows that the compound of the rational functions $F, \Phi$ is a rational function, and (since the expression within the braces is the inverse of an integral function) also expresses it in rational form.

4. Product of compositional powers of linear functions. The following theorem which expresses the product $L_{1}^{r} \times L_{2}^{s}$, where $L_{1}, L_{2}$ are linear functions, in rational form, is of fundamental importance. 
THEOREM XXII. If $L_{1}, L_{2}$ are linear functions, and $L_{12}=L_{1} \times L_{2}$,

$$
L_{1}^{r} \times L_{2}^{s}=L_{12}^{r+s-1} \cdot\left(L_{1}^{-(r-1)} \times L_{2}^{-(s-1)}\right),
$$

$r$ and s being positive integers.

To prove this, let

$$
L_{1(p)}(x)=\frac{1}{1-\alpha x}, \quad L_{2(p)}(x)=\frac{1}{1-\beta x} .
$$

Also, let $r_{n}^{B}$ denote the coefficient of $x^{n}$ in $(1-x)^{-r}$, and $\left(\begin{array}{l}r \\ n\end{array}\right)$, the usual binomial coefficient. Then

and

$$
\left(L_{1}^{r} \times L_{2}^{\prime}\right)_{(p)}(x)=\sum_{n}^{H} r_{n}^{H} \cdot s_{n}^{H}(\alpha \beta)^{n} x^{n},
$$

$$
\begin{aligned}
\left\{L_{12}^{r+s-1} \cdot\left(L_{1}^{-(r-1)} \times L_{2}^{-(o-1)}\right)\right. & { }_{(p)}(x) \\
& =\frac{1}{(1-\alpha \beta x)^{r+\sigma-1}} \cdot\left\{\sum\left(\begin{array}{c}
r-1 \\
n
\end{array}\right)\left(\begin{array}{c}
s-1 \\
n
\end{array}\right)(\alpha \beta)^{n} x^{n}\right\} .
\end{aligned}
$$

We have therefore to show that

$$
F(r, s, n) \equiv r_{n}^{H} \cdot s_{n}^{H}-\left(\begin{array}{c}
r+s-1 \\
1
\end{array}\right){ }_{r_{n-1}}^{H} \cdot s_{n-1}^{H}+\cdots=\left(\begin{array}{c}
r-1 \\
n
\end{array}\right)\left(\begin{array}{c}
s-1 \\
n
\end{array}\right) .
$$

To prove this, we note that when $n>r+s-1, F(r, s, n)$ is a polynomial in $n$ of degree $r+s-2$, and represents $\Delta_{n}^{r+s-1}\left(r_{n}^{H} \cdot s_{n}^{B}\right)$, where the operator $\Delta_{n}$ is defined by

$$
\Delta_{n} f(n)=f(n)-f(n-1) .
$$

Hence it follows that

$$
F(r, s, n)=0, \text { if } n \geqq r+s-1,
$$

since $r_{n}^{B} \cdot s_{n}^{B}$ is a polynomial of degree $r+s-2$ in $n$. Also, when $n<r+s-1$,

$$
\begin{aligned}
0 & \left.=\Delta_{n}^{r+s-1} \underset{\left(r_{n} \cdot s_{n}\right.}{H}\right) \\
& =F(r, s, n)+(-1)^{n+1}\left\{\begin{array}{c}
H \\
r_{-1} \cdot s_{-1}
\end{array} \cdot\left(\begin{array}{c}
r+s-1 \\
n+1
\end{array}\right)-r_{-2}^{H} \cdot s_{-2}^{H} \cdot\left(\begin{array}{c}
r+s-1 \\
n+2
\end{array}\right)+\cdots\right\} .
\end{aligned}
$$

Now $r_{-k}^{H}=0$ unless $k \geqq r$; hence the part within the braces will contain no significant terms if $r+s-1-n<r$, or if $r+s-1-n<s$. Hence $F(r, s, n)=0$ if $n$ is greater than either $r-1$ or $s-1$. Also when $n<r+s-1, F(r, s, n)$ is a polynomial in $r$, and also in $s$, of degree $n$, and we have just proved that it vanishes 
for the $n$ values $r-1=0,1,2, \cdots, n-1$, and also for the $n$ values $s-1=0,1, \cdots, n-1$. Hence we must have, identically.

$$
F(r, s, n)=\psi(n)\left(\begin{array}{c}
r-1 \\
n
\end{array}\right)\left(\begin{array}{c}
s-1 \\
n
\end{array}\right) .
$$

By putting $r=s=0$, we immediately find that $\psi(n)=1$. The theorem is thus established.

We may note that the relation $E^{2} \times E^{2}=E_{2} \cdot E^{2}$ (Example 3) is a very special case of this theorem.

5. Regular rational functions. By an elementary rational function, we shall mean a function which is either the inverse of a rational integral function, or is of the form $T \times L^{r}$, where $T$ is a level totient, and $L$ a linear function ( $r$ being a positive integer).

A rational function will be said to be regular, if no two of its distinct linear components have any common elements.

THEOREM XXIII. A regular rational function can be expressed as the compound of elementary rational functions.

For, let the integral component of the rational function $f$ be $L_{1}^{r_{1}} \cdot L_{2}^{r_{1}} \cdots L_{i}^{r_{i}}$, $L_{1}, L_{2}, \cdots, L_{i}$ being the distinct linear components of $f$. Also let

$$
f(p)(x)=\frac{\phi(x)}{\left(1-a_{1} x\right)^{r_{1}}\left(1-a_{2} x\right)^{r_{2}} \cdots\left(1-a_{i} x\right)^{r_{i}}},
$$

where $\phi(x)$ is a polynomial. Now, if the function $f$ is regular, then no two of the linear functions $L_{1}, L_{2}, \cdots, L_{i}$ have common elements, and therefore the quantities $a_{1}, a_{2}, \cdots, a_{i}$ will be unequal for every prime $p$. Hence for every prime $p$, we can express $f_{(p)}(x)$ in partial fractions in the form

$f(p)(x)=\phi_{1}(x)+\sum_{t=1}^{r_{1}} \frac{b_{1 t}}{\left(1-a_{1} x\right)^{t}}+\sum_{t=1}^{r_{2}} \frac{b_{2 t}}{\left(1-a_{2} x\right)^{t}}+\cdots+\sum_{t=1}^{r_{i}} \frac{b_{i t}}{\left(1-a_{i} x\right)^{t}}$,

$\phi_{1}(x)$ being a certain polynomial. That is to say, the function $f$ can be expressed as the compound of the inverse of an integral function, and functions of the type $T \times L_{j}^{t}$, where $T$ is a level totient, $j=1,2, \cdots, i$, and $t=1$, $2, \cdots, r_{j}$.

If, however, two of the functions $L$, say $L_{1}, L_{2}$, have identical elements for the base $q$ (so that $q$ is an irregular base of the function $f$ ), then $f_{(q)}(x)$ is not of the above form, the quantities $b_{1 s}, L_{2 t}\left(s=1,2, \cdots, r_{1} ; t=1,2, \cdots, r_{2}\right)$ now becoming infinite with certain mutual relations. We may however deal with this as a limiting case when $a_{2} \rightarrow a_{1}$. The limiting process will show 
generally that results proved for regular functions may be interpreted as true for irregular functions as well.

THEOREM XXIV. The product of regular rational functions is a rational function.

For, a regular function is the compound of elementary rational functions. Hence the product of two regular rational functions can be expressed as a compound of products of pairs of elementary rational functions. Now, the product of the inverse of an integral function by any other function is also the inverse of an integral function. And the product of the elementary functions $T_{1} \times L_{1}^{r}, T_{2} \times L_{2}^{s}$ is $T_{1} \times T_{2} \times f^{\prime}$, where $f^{\prime}$ is the rational function $L_{12}^{r+s-1} \cdot\left(L_{1}^{-(r-1)}\right.$ $\times L_{2}^{-(8-1)}$ ) (Theorem XXII). Also $T_{1}$ and $T_{2}$ being level totients, their product $T_{1} \times T_{2}$ is also a level totient, and $T_{1} \times T_{2} \times f^{\prime}$ is a rational function with the integral component $L_{12}^{r+s-1}$ (Example 11). Thus the product-function can be expressed as a compound of rational functions with known integral components, and is therefore a rational function.

To specify the integral component of the product-function, let the integral components of the regular functions $F_{1}, F_{2}$ be

$$
\begin{gathered}
L_{1}^{r_{1}} \cdot L_{2}^{r_{2}} \cdots L_{i}^{r_{i}}, \\
K_{1}^{s_{1}} \cdot K_{2}^{s_{2}} \cdots K_{i}^{s_{j}},
\end{gathered}
$$

respectively, the $L$ 's and $K$ 's being distinct linear functions. By what has preceded, $F_{1} \times F_{2}$ is the compound of rational functions, whose integral components are of the form

$L_{\mu \nu}^{\rho_{\mu}+\sigma_{\nu}-1}, \rho_{\mu} \leqq r_{\mu}, \sigma_{\nu} \leqq s_{\nu} ; L_{\mu \nu}=L_{\mu} \times K_{\nu} \quad(\mu=1,2, \cdots, i ; \nu=1,2, \cdots, j)$.

The integral component of $F_{1} \times F_{2}$ is therefore the least common composite of these (III §3); but this latter is evidently identical with the least common composite of the $i j$ functions $L_{\mu \nu}^{\gamma_{\mu}+s_{\nu}-1}$. This least common composite will coincide with the continued composite, if the $i j$ linear functions $L_{\mu \nu}$ are all distinct.

If $n_{1}, n_{2}, n$ are the degrees of the integral components of $F_{1}, F_{2}$ and $F_{1} \times F_{2}$, so that

$$
\begin{aligned}
& n_{1}=r_{1}+r_{2}+\cdots+r_{i}, \\
& n_{2}=s_{1}+s_{2}+\cdots+s_{i},
\end{aligned}
$$

it is easy to see that

$$
n=j n_{1}+i n_{2}-i j
$$


These results will hold generally, even when the functions are irregular, provided we consider that the product function has a certain number of elements in common with $E_{0}$ for the irregular bases.

6. Some special cases. (a) The rational form of $f \times L^{r}$, where $L$ is linear, and $f$ is rational. We can immediately find $f \times L$, where $L$ is linear; for

$$
(f \times L)_{(p)}(x)=f_{(p)}(l x) \text {, where } L_{(p)}=l .
$$

Since $L^{r}=L \times E^{r}$, it follows that it is sufficient to evaluate $f \times E^{r}$. If

then

$$
f_{(p)}(x)=1+a_{1} x+a_{2} x^{2}+\cdots,
$$

$$
\begin{aligned}
\left(f \times E^{r}\right)_{(p)}(x) & =1+a_{1} \cdot r_{1}^{H} x+a_{2} \cdot r_{2}^{H} x^{2}+\cdots \\
& =\frac{1}{(r-1) !} \frac{d^{r-1}}{d x^{r-1}}\left(x^{r-1} f_{(p)}(x)\right) .
\end{aligned}
$$

When $f$ is rational, this can be evaluated in finite terms. For example,

Hence

$$
E^{2} \times E^{2}=E^{2} \cdot E_{2} \text { (Example 3). }
$$

$$
\left(E^{2} \times E^{2}\right)_{(p)}(x)=\frac{1+x}{(1-x)^{3}} .
$$

Therefore

$$
\left(E^{2} \times E^{2} \times E^{2}\right)_{(p)}(x)=\frac{d}{d x}\left\{\frac{x(1+x)}{(1-x)^{3}}\right\}=\frac{1+4 x+x^{2}}{(1-x)^{4}},
$$

so that

$$
E^{2} \times E^{2} \times E^{2}=E^{4} \cdot\left(E^{-2} \times E^{-2}\right)=E^{-1} \cdot\left(E^{3} \times E^{3}\right)(\text { Theorem XXII) } .
$$

This gives a second proof of Liouville's theorem (Example 4):

$$
\sum_{d / N}\{\tau(d)\}^{3}=\left\{\sum \tau(d)\right\}^{2} .
$$

(b) Product of integral quadratic functions. If the $L$ 's are linear functions, $L_{i j}=L_{i} \times L_{j}$, and $L_{1234}=L_{1} \times L_{2} \times L_{3} \times L_{4}$, then

$$
\left(L_{1} \cdot L_{2}\right) \times\left(L_{3} \cdot L_{4}\right)=L_{13} \cdot L_{14} \cdot L_{23} \cdot L_{24} \cdot \operatorname{conv} L_{1234}^{-1} \text {. }
$$

This may be proved directly from the generating series, or by the method of grouping factors according to their greatest common divisor and least common multiple, employed in Example 9. Let the function $f(N)$ be defined by

$$
f(N)=\sum L_{1}\left(d_{1}\right) L_{2}\left(\frac{N}{d_{1}}\right) L_{3}\left(d_{2}\right) L_{4}\left(\frac{N}{d_{2}}\right),
$$


where the summation is for all pairs of numbers $d_{1}, d_{2}$ having the least common multiple $N$. We shall evaluate $f(N)$ in two different ways. First group the terms in the sum according to the greatest common divisor $\delta$ of $d_{1}, d_{2}$. Writing

$$
d_{1}=\delta t_{1} ; d_{2}=\delta t_{2}
$$

so that

we have

$$
N=\delta t_{1} t_{2}
$$

$$
\begin{aligned}
f(N)=\sum L_{1}\left(d_{1}\right) L_{2}\left(\frac{N}{d_{1}}\right) L_{3}\left(d_{2}\right) L_{4}\left(\frac{N}{d_{2}}\right) & =\sum L_{1}(\delta) L_{1}\left(t_{1}\right) L_{2}\left(t_{2}\right) L_{3}(\delta) L_{3}\left(t_{2}\right) L_{4}\left(t_{1}\right) \\
& =\sum L_{13}(\delta) L_{14}\left(t_{1}\right) L_{23}\left(t_{2}\right) \\
& =\left\{L_{13} \cdot\left(L_{14} \oplus L_{23}\right)\right\}(N) \\
& =\left\{L_{13} \cdot L_{14} \cdot L_{23} \cdot \operatorname{conv} L_{1234}^{-1}\right\}(N)
\end{aligned}
$$

(Theorem XV).

Secondly, let us evaluate $f \cdot L_{24}$. We have

$$
\begin{aligned}
f(\delta) L_{24}\left(\frac{N}{\delta}\right) & =\sum L_{1}\left(\delta_{1}\right) L_{2}\left(\frac{\delta}{\delta_{1}}\right) L_{3}\left(\delta_{2}\right) L_{4}\left(\frac{\delta}{\delta_{2}}\right) L_{24}\left(\frac{N}{\delta}\right) \\
& =\sum L_{1}\left(\delta_{1}\right) L_{2}\left(\frac{N}{\delta_{1}}\right) L_{3}\left(\delta_{2}\right) L_{4}\left(\frac{N}{\delta_{2}}\right),
\end{aligned}
$$

the summation extending to all pairs of numbers $\delta_{1}, \delta_{2}$ having the least common multiple $\delta$. Summing both sides of this over all divisors $\delta$ of $N$, we see that $\left(f \cdot L_{24}\right)(N)$ is equal to

$$
\sum L_{1}\left(\delta_{1}\right) L_{2}\left(\frac{N}{\delta_{1}}\right) L_{3}\left(\delta_{2}\right) L_{4}\left(\frac{N}{\delta_{2}}\right),
$$

summed for every pair of divisors $\delta_{1}, \delta_{2}$ of $N$, that is, to $\left\{\left(L_{1} \cdot L_{2}\right) \times\left(L_{3} \cdot L_{4}\right)\right\}(N)$. Combining the two relations, we have immediately

$$
\left(L_{1} \cdot L_{2}\right) \times\left(L_{3} \cdot L_{4}\right)=L_{13} \cdot L_{14} \cdot L_{23} \cdot L_{24} \cdot \operatorname{conv} L_{1234}^{-1} .
$$

As a special case of this, we have the result already proved (Example 9):

$$
\sigma_{a} \times \sigma_{b}=E \cdot I_{a} \cdot I_{b} \cdot I_{a+b} \cdot I_{a+b, 2}^{-1} .
$$

As another special case:

$$
\begin{aligned}
\left(L_{1} \cdot L_{2}\right) \times\left(L_{1} \cdot L_{2}\right) & =L_{11} \cdot L_{22} \cdot L_{12}^{2} \cdot \operatorname{conv}\left(L_{12} \times L_{12}\right)^{-1} \\
& =L_{11} \cdot L_{22} \cdot L_{12} \cdot\left(L_{12} \times \lambda^{-1}\right)
\end{aligned}
$$


SeCtion IV. The CARDINAL, PRINCIPAL, AND SEMI-PRINCIPAL FUNCTIONS

1. Derivates of a function of $r$ arguments. The derivate of the multiplicative function $f\left(M_{1}, M_{2}, \cdots, M_{r}\right)$ with respect to any subset $M_{1}$, $M_{2}, \cdots, M_{i}$ of its arguments, is defined to be the function of the remaining $r-i$ arguments, obtained on putting $M_{1}=M_{2}=\cdots=M_{i}=1$ in $f\left(M_{1}\right.$, $\left.M_{2}, \cdots, M_{r}\right)$. This derivate is clearly a multiplicative function, and may be denoted by the symbol $D_{M_{1} M_{2} \ldots M_{i}}(f)$.

If $f_{(p)}\left(x_{1}, x_{2}, \cdots, x_{r}\right)$ is the generating series of $f$ to the base $p$, the generating series of $D_{M_{1} M_{2} \ldots M_{i}}(f)$ to the same base is clearly $f_{(p)}(0,0, \cdots$, $\left.x_{i+1}, x_{i+2}, \cdots, x_{r}\right)$. From this we immediately deduce then

THEOREM XXV. If $f$, $\phi$ be two multiplicative functions of $M_{1}, M_{2}, \cdots, M_{r}$,

$$
\begin{aligned}
D_{M_{1} M_{2} \cdots M_{i}}(f) \times D_{M_{1} M_{2} \cdots M_{i}}(\phi) & =D_{M_{1} M_{2} \cdots M_{i}}(f \times \phi), \\
D_{M_{1} M_{2} \cdots M_{i}}(f) \cdot D_{M_{1} M_{2} \cdots M_{i}}(\phi) & =D_{M_{1} M_{2} \cdots M_{i}}(f \cdot \phi), \\
D_{M_{1} M_{2} \cdots M_{i}}\left(f^{-1}\right) & =\left\{D_{M_{1} M_{2} \cdots M_{i}}(f)\right\}^{-\mathrm{i}} .
\end{aligned}
$$

Or briefly, the product or compound of derivates is the derivate of the product or compound, and the derivate of the inverse is the inverse of the derivate.

It will be seen in the next section, that the derivates also possess a similar property with respect to the compounding operation.

Theorem XXVI. The necessary and sufficient condition that a multiplicafive function $f$ of the $r$ arguments $M_{1}, M_{2}, \cdots, M_{r}$, be also a multiplicative tunction of a subset $M_{1}, M_{2}, \cdots, M_{i}$ of its arguments, is

$$
D_{M_{1} M_{2} \cdots M_{i}}(f) \equiv E\left(M_{i+1}, M_{i+2}, \cdots, M_{r}\right) .
$$

The condition is obviously necessary, since a multiplicative function of $M_{1}, M_{2}, \cdots, M_{i}$ must be equal to 1 , when $M_{1}=M_{2}=\cdots=M_{i}=1$. To prove that it is sufficient, we observe that, when $D_{M_{1} M_{2} \cdots M_{i}}(f)$ $=E\left(M_{i+1}, \cdots, M_{r}\right)$, the value of $f\left(M_{1}, M_{2}, \cdots, M_{r}\right)$ is not affected either by introducing into, or removing from, the last $r-i$ arguments, such factors as are relatively prime to $M_{1} M_{2} \cdots M_{i}$. For, if $p_{a}$ be any prime which does not divide $M_{1} M_{2} \cdots M_{r}$,

$$
\begin{aligned}
& f\left(M_{1}, \cdots, M_{i}, M_{i+1} p^{\alpha}, M_{i+2} p^{\beta}, \cdots\right) \\
= & f\left(M_{1}, M_{2}, \cdots, M_{r}\right) \times f\left(1,1, \cdots, p^{\alpha}, p^{\beta}, \cdots\right) \\
= & f\left(M_{1}, M_{2}, \cdots, M_{r}\right)=f\left(M_{1}, M_{2}, \cdots, M_{i}, M_{i+1} p^{\alpha^{\prime}}, M_{i+2} p^{\beta^{\prime}}, \cdots\right) .
\end{aligned}
$$

Hence, writing 


$$
\begin{aligned}
M_{i+1}^{\prime} & =M_{i+1} p^{\alpha} ; \quad M_{i+2}^{\prime}=M_{i+2} p^{\beta} ; \text { etc. } \\
\alpha^{\prime}-\alpha & =a ; \quad \beta^{\prime}-\beta=b ; \text { etc. }
\end{aligned}
$$

we have

$f\left(M_{1}, M_{2}, \cdots, M_{i}, M_{i+1}^{\prime}, \cdots, M_{r}^{\prime}\right)=f\left(M_{1}, \cdots, M_{i}, M_{i+1}^{\prime} p^{a}, M_{i+2}^{\prime} p^{b}, \cdots\right)$, where $p$ is any prime not occurring in $M_{1}, M_{2}, \cdots, M_{i}$. This proves our statement.

To prove that $f\left(M_{1}, M_{2}, \cdots, M_{r}\right)$ is a multiplicative function of $M_{1}, M_{2}, \cdots, M_{i}$, that is, that, whenever $M_{1} M_{2} \cdots M_{i}$ is relatively prime to $N_{1} N_{2} \cdots N_{i}$,

$$
\begin{aligned}
f\left(M_{1} N_{1}, M_{2} N_{2}, \cdots,\right. & \left.M_{i} N_{i}, M_{i+1}, \cdots, M_{r}\right) \\
& =f\left(M_{1}, M_{2}, \cdots, M_{r}\right) \times f\left(N_{1}, \cdots, N_{i}, M_{i+1}, \cdots, M_{r}\right),
\end{aligned}
$$

suppose that $M_{i+k}^{\prime}, M_{i+k}^{\prime \prime}$ are the maximum factors of $M_{i+k}$, which contain only prime factors occurring in $M_{1} M_{2} \cdots M_{i}, N_{1} N_{2} \cdots N_{i}$, respectively. It follows that $M_{i+k}^{\prime}, M_{i_{+k}^{\prime}}^{\prime \prime}$ are relatively prime, and therefore $M_{i+k}=$ $M_{i+k}^{\prime} M_{i+k}^{\prime \prime} T_{i+k}$, where $T_{i+k}$ is relatively prime to $M_{1} M_{2} \cdots M_{i} N_{1} N_{2} \cdots N_{i}$. Now, since $M_{i+k}^{\prime}, M_{i+k}^{\prime \prime}$ are respectively prime to $N_{1} N_{2} \cdots N_{i}, M_{1} M_{2} \cdots M_{i}$, it follows that

$$
\begin{aligned}
f\left(M_{1}, M_{2}, \cdots, M_{r}\right) & =f\left(M_{1}, M_{2}, \cdots, M_{i}, M_{i+1}^{\prime}, M_{i+2}^{\prime}, \cdots, M_{r}^{\prime}\right), \\
f\left(N_{1}, N_{2}, \cdots, N_{i}, M_{i+1}, \cdots, M_{r}\right) & =f\left(N_{1}, N_{2}, \cdots, N_{i}, M_{i+1}^{\prime \prime}, M_{i+2}^{\prime \prime}, \cdots, M_{r}^{\prime \prime}\right) .
\end{aligned}
$$

Now, however, $M_{1} M_{2} \cdots M_{i} M_{i+1}^{\prime} \cdots M_{r}^{\prime}$ is relatively prime to $N_{1} N_{2} \cdots$ $N_{i} M_{i^{\prime}+1}^{\prime \prime} \cdots M_{r}^{\prime \prime}$. Hence, since $f\left(M_{1}, M_{2}, \cdots, M_{r}\right)$ is a multiplicative function of its $r$ arguments, it follows that the product of the left sides is equal to

$$
f\left(M_{1} N_{1}, M_{2} N_{2}, \cdots, M_{i} N_{i}, M_{i+1}^{\prime} M_{i+1}^{\prime \prime}, \cdots, M_{r}^{\prime} M_{r}^{\prime \prime}\right) .
$$

Here, the factors $T_{i+k}$, which are relatively prime to the first $i$ arguments of this function, may be introduced into the last $r-i$ arguments, without affecting the value. Thus

$$
\begin{aligned}
& f\left(M_{1}, \cdots, M_{i}, M_{i+1}, \cdots, M_{r}\right) f\left(N_{1}, \cdots, N_{i}, M_{i+1}, \cdots, M_{r}\right) \\
& \quad=f\left(M_{1} N_{1}, \cdots, M_{i} N_{i}, T_{i+1} M_{i+1}^{\prime} M_{i+1}^{\prime \prime}, \cdots\right) \\
& \quad=f\left(M_{1} N_{1}, \cdots, M_{i} N_{i}, M_{i+1}, \cdots, M_{r}\right)
\end{aligned}
$$

establishing the multiplicative property in respect to $M_{1}, M_{2}, \cdots, M_{i}$. 
As an illustration of the theorem, any multiplicative function $f(g)$ of the greatest common divisor $g$ of $M_{1}, M_{2}, \cdots, M_{r}$ is seen to be a multiplicative function, not only of $M_{1}, M_{2}, \cdots, M_{r}$, but of any subset of them as well; for when any of the arguments $M_{1}, M_{2}, \cdots, M_{r}$ becomes equal to unity, $g$ and therefore also $f(g)$ take the value unity.

2. Cardinal functions of $r$ arguments. A cardinal function of $M_{1}, M_{2}, \cdots$, $M_{r}$ is defined to be a function $f$, all of whose derivates are identical with $E_{0}$; it is clearly necessary and sufficient for this that the $r$ derivates $D_{M_{i}}(f)$ $(i=1,2, \cdots, r)$ be identical with $E_{0}$. This implies that the generating series $\left(f_{p}\right)\left(x_{1}, x_{2}, \cdots, x_{r}\right)$ of a cardinal function $f$, is of the form $1+x_{1} x_{2} \cdots x_{r}$ $F\left(x_{1}, x_{2}, \cdots, x_{r}\right)$, where $F\left(x_{1}, x_{2}, \cdots, x_{r}\right)$ is some power series. The arithmetical property of the cardinal function $f$, implied by this, is evidently that $f\left(M_{1}, M_{2}, \cdots, M_{r}\right)=0$, whenever $M_{i}$ admits a factor relatively prime to $M_{j}$, or in other words, that $f\left(M_{1}, M_{2}, \cdots, M_{r}\right)=0$, unless each of the arguments has the same distinct prime factors.

From Theorem XXIV, we now have, immediately,

THEOREM XXVII. The product of a cardinal function and an arbitrary function of the same arguments, is also a cardinal function. The composite of a cardinal function and an arbitrary function $f$ has all its derivates identical with the corresponding derivates of $f$. Lastly, the inverse of a cardinal function is also a cardinal function.

To prove the last statement, let $F$ be a cardinal function. Then,

$$
\begin{aligned}
D_{M_{i}}(F) \cdot D_{M_{i}}\left(F^{-1}\right) & =D_{M_{i}}\left(F \cdot F^{-1}\right) \\
& =D_{M_{i}}\left(E_{0}\right)=E_{0} .
\end{aligned}
$$

But, since $F$ is a cardinal function, $D_{M_{i}}(F)=E_{0}$. Hence $D_{M_{i}}\left(F^{-1}\right)=E_{0}$, or $F^{-1}$ is also a cardinal function.

The converse of this theorem is of great importance; it is as follows:

Theorem XXVIII. If $\phi_{1}, \phi_{2}$ are functions of $M_{1}, M_{2}, \cdots, M_{r}$, whose corresponding derivates are identical, then each of them is the composite of the other with a cardinal function; also, each is the product of the other and the integral of a cardinal function.

For, if

$$
\phi_{1}=\phi_{2} \cdot f=\phi_{2} \times(F \cdot E),
$$

then 


$$
D_{M_{i}}\left(\phi_{1}\right)=D_{M_{i}}\left(\phi_{2}\right) \cdot D_{M_{i}}(f)=D_{M_{i}}\left(\phi_{2}\right) \times\left(D_{M_{i}}(F) \cdot E\right) \quad\left(\text { since } D_{M_{i}}(E)=E\right) \text {. }
$$

Since $D_{M_{i}}\left(\phi_{1}\right)=D_{M_{i}}\left(\phi_{2}\right)$ by hypothesis, it follows that

$$
\begin{aligned}
D_{M_{i}}(f) & =E_{0}, \\
D_{M_{i}}(F) \cdot E & =E \text {, or } D_{M_{i}}(F)=E_{0} .
\end{aligned}
$$

Thus both $f$ and $F$ are cardinal functions.

CoROllary 1. If $\phi_{1}, \phi_{2}$ are functions of $M_{1}, M_{2}, \cdots, M_{r}$ which are known to be equal to one another, whenever two of the arguments $M_{1}, M_{2}, \cdots, M_{r}$ are relatively prime, it is thereby implied that the functions are also equal when any of the arguments is equal to unity. Thus $\phi_{1}, \phi_{2}$ have identical derivates, and the theorem applies to them.

COROLlaRY 2. The most general function $f\left(M_{1}, M_{2}, \cdots, M_{r}\right)$ with the property of being a multiplicative function of every subset of its arguments is the integral (II §3) of an arbitrary cardinal function.

3. Cardinal functions of a matrix-set of arguments. A function $f$ of the matrix-set of $r \times s$ arguments $\left|M_{i 1}, M_{i 2}, \cdots, M_{i r}\right|(i=1,2, \cdots, s)$, is said to be a cardinal function, if the derivate of $f$ with respect to the $s$ arguments of any column (or, briefly, if each column-derivate of $f$ ) is the function $E_{0}$. This reduces to our earlier definition for $s=1$. It follows that a cardinal function of the matrix-set $\left|M_{i 1}, M_{i 2}, \cdots, M_{i r}\right|(i=1,2, \cdots, s)$ vanishes if any of the $r$ column-products $M_{1 j} M_{2 j} \cdots M_{s j}(j=1,2, \cdots, r)$ contains a prime factor not dividing one of the others. We have the following obvious analogue of Theorem XXVIII:

THEOREM XXIX. If $\phi_{1}, \phi_{2}$ are two functions of the matrix set $\mid M_{i 1}$, $M_{i 2}, \cdots, M_{i r} \mid(i=1,2, \cdots, s)$, which have identical column-derivates, then each is the composite of the other with a cardinal function, and also the product of the other with the integral of a cardinal function of the matrix-set.

The proof is on the same lines as the proof of Theorem XXVIII. As in Corollary 1 of that theorem, this also applies to two functions of the matrixset, which are known to be equal to each other, whenever any two columnproducts are relatively prime.

From the definition of the cardinal function of a matrix-set $\left|M_{i j}\right|$, it is easy to specify the peculiarity of its generating series to any base. Let $x_{i j}$ be the variable corresponding to the argument $M_{i j}$. Since every column-derivate of the cardinal function is $E_{0}$, it follows that the generating series must reduce to 1 , if we put the variables corresponding to any column equal to zero. Thus the peculiarity of the generating series is that every non-vanishing 
term of it contains at least one representative from each column of the matrix $\left|x_{i j}\right|$, or in other words, the terms which involve none of the variables of a column vanish.

4. Principal functions. These constitute a very important subclass of cardinal functions. The function $f\left(M_{1}, M_{2}, \cdots, M_{r}\right)$ is called a principal function, if it vanishes whenever two of its arguments are unequal. The function

$$
\psi(M)=f(M, M, \cdots, M)
$$

is called the function of one argument equivalent to the principal function $f$. It is clear that $\psi$ determines $f$ completely; for, if

$$
\psi_{(p)}(x)=1+a_{1} x+a_{2} x^{2}+\cdots,
$$

then

$$
\begin{aligned}
f_{(p)}\left(x_{1}, x_{2}, \cdots, x_{r}\right)=1 & +a_{1}\left(x_{1} x_{2} \cdots x_{r}\right) \\
& +a_{2}\left(x_{1} x_{2} \cdots x_{r}\right)^{2}+\cdots .
\end{aligned}
$$

This will be indicated by writing

$f=\operatorname{princ}_{r} \psi(f$ is the principal function of $r$ arguments, equivalent to $\psi$ ).

More generally, a function $f$ of the matrix-set $\left|M_{i 1}, M_{i 2}, \cdots, M_{i r}\right|(i=1$, $2, \cdots, s)$ will be called a principal function, if it vanishes whenever two arguments in the same row are unequal. The function $\psi\left(M_{1}, M_{2}, \cdots, M_{s}\right)$ to which $f$ reduces when $M_{i 1}=M_{i 2}=\cdots=M_{i r}=M_{i}(i=1,2, \cdots, s)$, is called the function of $s$ arguments equivalent to the principal function $f$. It is clear that $\psi$ determines $f$, and that the generating series of $f$ is obtained from $\psi_{(p)}\left(x_{1}, x_{2}, \cdots, x_{s}\right)$, by substituting $x_{i 1} x_{i 2} \cdots x_{i r}$ for $x_{i}(i=1,2, \cdots, s)$. We also call $f$ the principal function of $r \times s$ arguments equivalent to $\psi\left(M_{1}\right.$, $M_{2}, \cdots, M_{s}$ ), and denote it by princ $r$.

THEOREM XXX. If $\psi, \psi^{\prime}$ are functions of $s$ arguments, and princ $\psi$, princ $\psi^{\prime}$ the equivalent principal functions of $r \times s$ arguments, then

$$
\operatorname{princ} \psi \cdot \operatorname{princ} \psi^{\prime}=\operatorname{princ}\left(\psi \cdot \psi^{\prime}\right),
$$

$$
(\operatorname{princ} \psi)^{-1}=\operatorname{princ}\left(\psi^{-1}\right) .
$$

These relations follow immediately from the fact that the generating series of princ $\psi$ is obtained from the generating series of $\psi$ by substituting $x_{i 1} x_{i 2} \cdots x_{i r}$ for $x_{i}(i=1,2, \cdots, s)$.

5. Functions of the greatest common divisor (g.c.d.). We prove the following theorem. 
TheORem XXXI. The necessary and sufficient condition that $f\left(M_{1}\right.$, $\left.M_{2}, \cdots, M_{r}\right)$ be a function $\psi(g)$ of the greatest common divisor $g$ of $M_{1}$, $M_{2}, \cdots, M_{r}$, is that $f$ be the integral of the principal function of $r$ arguments, equivalent to $\left(\psi \cdot E^{-1}\right)(M)$.

The necessary and sufficient condition that $f\left(\left|M_{i 1}, M_{i 2}, \cdots, M_{i r}\right|\right)(i=1$, $2, \cdots, s)$ be a function $\psi\left(g_{1}, g_{2}, \cdots, g_{s}\right)$ of the $g . c . d . ' s g_{i}$ of $M_{i 1}, M_{i 2}, \cdots, M_{i r}$, is that $f$ be the integral of the principal function of $r \times s$ arguments, equivalent to $\left(\psi \cdot E^{-1}\right)\left(M_{1}, M_{2}, \cdots, M_{s}\right)$.

This theorem illustrates Theorem XXIX; for, if two column-products are relatively prime, it is clear that $g_{1}, g_{2}, \cdots, g_{8}$ all become unity, and so $f=1=E$. Hence, by Theorem XXIX, $f$ is the composite of $E$ and a cardinal function. The present theorem identifies the cardinal function as a certain principal function.

To prove the first part, let

$$
f_{(p)}\left(x_{1}, x_{2}, \cdots, x_{r}\right)=\sum a\left(m_{1}, m_{2}, \cdots, m_{r}\right) x_{1}^{m_{1}} x_{2}^{m_{2}} \cdots x_{r}^{m_{r}},
$$

so that

$$
\left(f \cdot E^{-1}\right)_{(p)}\left(x_{1}, x_{2}, \cdots, x_{r}\right)=\sum b\left(m_{1}, m_{2}, \cdots, m_{r}\right) x_{1}^{m_{1}} x_{2}^{m_{2}} \cdots x_{r}^{m_{r}} \text {, }
$$

where

$$
\begin{aligned}
b\left(m_{1}, m_{2}, \cdots, m_{r}\right)= & a\left(m_{1}, m_{2}, \cdots, m_{r}\right)-\sum a\left(m_{1}-1, m_{2}, \cdots, m_{r}\right) \\
& +\sum a\left(m_{1}-1, m_{2}-1, m_{3}, \cdots, m_{r}\right)-\cdots,
\end{aligned}
$$

terms with negative suffixes not appearing in the summations. Since $f$ is given to be a function of the g.c.d. of its arguments, $a\left(m_{1}, m_{2}, \cdots, m_{r}\right)$ must depend only on the least of its suffixes. Suppose that $m$ is the least of $m_{1}, m_{2}, \cdots, m_{r}$, and that

$$
\begin{gathered}
m_{1}=m_{2}=\cdots=m_{i}=m ; m_{i+1}, m_{i+2}, \cdots, m_{r}>m ; \\
a\left(m_{1}, m_{2}, \cdots, m_{r}\right)=A(m) .
\end{gathered}
$$

Now group the expression for $b\left(m_{1}, m_{2}, \cdots, m_{r}\right)$ in such a way that in each group the values of the first $i$ suffixes remain the same. If $m=0$, then, since negative suffixes are not permitted, there will be only one group, and the value of $b\left(m_{1}, m_{2}, \cdots, m_{r}\right)$ will be 1 , or 0 , according as all the $m^{\prime}$ 's do, or do not, vanish. (This result might of course have been anticipated, since we know that $f \cdot E^{-1}$ must be a cardinal function.) If $m>0$, then there are $2^{i}$ groups of terms, and we have

$$
b\left(m_{1}, m_{2}, \cdots, m_{r}\right)=A(m)(1-1)^{r-i}-\left(\begin{array}{c}
i \\
1
\end{array}\right) A(m-1)(1-1)^{r-i}
$$




$$
\begin{aligned}
& +\left(\begin{array}{c}
i \\
2
\end{array}\right) A(m-1)(1-1)^{r-i}-\text { etc. } \\
= & \{A(m)-A(m-1)\}(1-1)^{r-i} .
\end{aligned}
$$

This vanishes unless $r=i$, and is then equal to $A(m)-A(m-1)$. Thus $b\left(m_{1}, m_{2}, \cdots, m_{r}\right)$ vanishes unless all the $m$ 's are equal, and is equal to $A(m)-A(m-1)$, if $m_{1}=m_{2}=\cdots=m_{r}=m$. Now the series

$$
1+\sum_{m=1}\{A(m)-A(m-1)\} x^{m}
$$

is evidently the generating series to the base $p$, of the function $\psi \cdot E^{-1}$. Thus $f \cdot E^{-1}$ is the principal function equivalent to the function $\left(\psi \cdot E^{-1}\right)(M)$.

The same method will apply to the case of a matrix-set of arguments. For convenience of writing we may take $s=2$, so that

$$
f\left(\left|\begin{array}{c}
M_{11}, M_{12}, \cdots, M_{1 r} \\
M_{21}, M_{22}, \cdots, M_{2 r}
\end{array}\right|\right)=\psi\left(g_{1}, g_{2}\right) .
$$

Let

$$
\begin{aligned}
\psi_{(p)}(x, y) & =\sum A(m, n) x^{m} y^{n}, \\
f_{(p)}\left(\begin{array}{c}
x_{1}, x_{2}, \cdots, x_{r} \\
y_{1}, y_{2}, \cdots, y_{r}
\end{array}\right) & =\sum a\left(\begin{array}{c}
m_{1}, m_{2}, \cdots, m_{r} \\
n_{1}, n_{2}, \cdots, n_{r}
\end{array}\right) x_{1}^{m_{1}} x_{2}^{m_{2}} \cdots x_{r}^{m_{r}} y_{1}^{n_{1}} y_{2}^{n_{2}} \cdots y_{r}^{n_{r}},
\end{aligned}
$$

so that, as before,

$$
a\left(\begin{array}{c}
m_{1}, m_{2}, \cdots, m_{r} \\
n_{1}, n_{2}, \cdots, n_{r}
\end{array}\right)=A(m, n),
$$

where $m$ is the least of $m_{1}, m_{2}, \cdots, m_{r}$, and $n$, the least of $n_{1}, n_{2}, \cdots, n_{r}$. If

$$
\begin{aligned}
&\left(f \cdot E^{-1}\right)_{(p)}\left(\begin{array}{c}
x_{1}, x_{2}, \cdots, \\
y_{1}, y_{2}, \cdots, y_{r}
\end{array}\right) \\
& \quad=\sum b\left(\begin{array}{c}
m_{1}, m_{2}, \cdots, m_{r} \\
n_{1}, n_{2}, \cdots, n_{r}
\end{array}\right) x_{1}^{m_{1}} x_{2}^{m_{2}} \cdots x_{r}^{m_{r}} y_{1}^{n_{1}} \cdots y_{r}^{n_{r}},
\end{aligned}
$$

then

$$
\begin{gathered}
b\left(\begin{array}{c}
m_{1}, m_{2}, \cdots, m_{r} \\
n_{1}, n_{2}, \cdots, n_{r}
\end{array}\right)=a\left(\begin{array}{r}
m_{1}, m_{2}, \cdots, m_{r} \\
n_{1}, n_{2}, \cdots, n_{r}
\end{array}\right)-\sum a\left(\begin{array}{rr}
m_{1}-1, m_{2}, \cdots, m_{r} \\
n_{1}, & n_{2}, \cdots, n_{r}
\end{array}\right) \\
+\sum a\left(\begin{array}{rr}
m_{1}-1, m_{2}-1, m_{3}, \cdots, m_{r} \\
n_{1}, \quad n_{2}, \quad n_{3}, \cdots, n_{r}
\end{array}\right)-\text { etc. }
\end{gathered}
$$


where the $k$ th summation sign indicates that every set of $k$ suffixes from among $m_{1}, m_{2}, \cdots, m_{r}, n_{1}, \cdots, n_{r}$ are to be diminished by unity, terms with negative suffixes being omitted.

As before, let

$$
\begin{aligned}
m_{1} & =m_{2}=\cdots=m_{i}=m ; m_{i+1}, m_{i+2}, \cdots, m_{r}>m ; \\
n_{1} & =n_{2}=\cdots=n_{i}=n ; \quad n_{j+1}, n_{j+2}, \cdots, n_{r}>n .
\end{aligned}
$$

Group the terms in the expression for

$$
b\left(\begin{array}{c}
m_{1}, \cdots, m_{r} \\
n_{1}, \cdots, n_{r}
\end{array}\right)
$$

in such a way, that, in each group, the first $i$ suffixes in the top row and the first $j$ suffixes in the bottom row have the same values. Then it is easy to see that, on evaluating each group separately, we obtain

$$
\begin{aligned}
b\left(\begin{array}{c}
m_{1}, m_{2}, \cdots, m_{r} \\
n_{1}, n_{2}, \cdots, n_{r}
\end{array}\right)= & (1-1)^{2 r-i-j}\left\{A(m, n)+A(m-1, n) \sum_{\mu=1}^{i}(-1)^{\mu}\left(\begin{array}{c}
i \\
\mu
\end{array}\right)\right. \\
& +A(m, n-1) \sum_{\nu=1}^{j}(-1)^{\nu}\left(\begin{array}{l}
j \\
\nu
\end{array}\right) \\
& \left.+A(m-1, n-1) \sum(-1)^{\mu+\nu}\left(\begin{array}{l}
i \\
\mu
\end{array}\right)\left(\begin{array}{l}
j \\
\nu
\end{array}\right)\right\} \\
= & (1-1)^{2 r-i-j}\{A(m, n)-A(m-1, n)-A(m, n-1) \\
& +A(m-1, n-1)\} .
\end{aligned}
$$

Thus

$$
b_{r}^{r}\left(\begin{array}{c}
m_{1}, m_{2}, \cdots, m_{r} \\
n_{1}, n_{2}, \cdots, n_{r}
\end{array}\right)
$$

is equal to zero, if two of the $m$ 's or two of the $n$ 's are unequal, and to $A(m, n)-A(m-1, n)-A(m, n-1)+A(m-1, n-1)$, if $m_{1}=m_{2}=\cdots=m_{r}$ $=m ; n_{1}=n_{2}=\cdots=n_{r}=n$. Thus $f \cdot E^{-1}$ is the principal function equivalent to $\psi \cdot E^{-1}$.

COROLLARY 1. The generating function to the base $p$ of the multiplicative function of $M_{1}, M_{2}, \cdots, M_{r}$, which is equal to their g.c.d., must, by the present theorem, be

$$
\frac{1-x_{1} x_{2} \cdots x_{r}}{\left(1-x_{1}\right)\left(1-x_{2}\right) \cdots\left(1-x_{r}\right)\left(1-p x_{1} x_{2} \cdots x_{r}\right)}
$$


This may be easily verified by direct expansion.

Corollary 2. The composite of $F\left(M_{1}, M_{2}, \cdots, M_{r}\right)$ and a principal function can be expressed as the composite of $F \cdot E^{-1}$ and a function $K(g)$ of the g.c.d. $g$ of $M_{1}, M_{2}, \cdots, M_{r}$.

For

$$
\begin{aligned}
F \cdot \operatorname{princ} \psi & =F \cdot E^{-1} \cdot E \cdot \operatorname{princ}\left(\psi \cdot E \cdot E^{-1}\right) \\
& =F \cdot E^{-1} \cdot(\psi \cdot E)(g) .
\end{aligned}
$$

As an illustration we may show that

$$
\sum \phi_{k}\left(\frac{M}{\delta_{1}}\right) \phi_{k}\left(\frac{N}{\delta_{2}}\right) \tau\left(\delta_{12}\right)=M^{k} N^{k} \frac{\sigma_{2 k}(g)}{g^{2 k}},
$$

where $\phi_{k}$ is the Jordan function, and the summation is extended to all divisors $\delta_{1}$ of $M$, and $\delta_{2}$ of $N, \delta_{12}$ being the g.c.d. of $\delta_{1}, \delta_{2}$, and $g$ the g.c.d. of $M, N$. For, if we write $\Phi_{k}(M, N)=I_{k}(M, N) \cdot E^{-1}(M, N)$, so that $\Phi_{k}(M, N)=\phi_{k}(M) \phi_{k}(N)$, the left side is

$$
\begin{aligned}
\Phi_{k} \cdot E^{2}(g) & =\Phi_{k} \cdot E \cdot \operatorname{princ}\left(E^{2} \cdot E^{-1}\right) \\
& =I_{k} \cdot \operatorname{princ} E \\
& =M^{k} N^{k} \sum \delta^{-2 k} \text { summed for common divisors } \delta, \\
& =M^{k} N^{k} \sigma_{2 k}(g) / g^{2 k} .
\end{aligned}
$$

6. Semiprincipal functions and functions of the least common multiple (1.c.m.). The semiprincipal functions are analogous to the principal functions, in that they are determined by functions of a smaller number of arguments; but they are not cardinal functions.

The function $f\left(M_{1}, M_{2}, \cdots, M_{r}\right)$ is said to be the semiprincipal function equivalent to $\psi(M)$, if, for any prime $p, f\left(p^{t_{1}}, p^{t_{2}}, \cdots, p^{t_{r}}\right)$ vanishes, whenever any two non-zero exponents $t$ are unequal, and is equal to $(-1)^{r-\mu+1} \psi\left(p^{r}\right)$, if $\mu$ of the exponents $t$ are zero, and the remaining are equal to $\tau$. Otherwise expressed, the semiprincipal function of $M_{1}, M_{2}, \cdots, M_{r}$, equivalent to $\psi(M)$, vanishes unless the g.c.d. $g_{i j}$ of $M_{i}, M_{j}$ is relatively prime both to $M_{i} / g_{i j}$, and to $M_{i} / g_{i j}(i, j=1,2, \cdots, r)$, and is equal, when this condition is satisfied, to

$$
\frac{\psi(l) E_{-1}\left(M_{1}\right) E_{-1}\left(M_{2}\right) \cdots E_{-1}\left(M_{r}\right)}{E_{-1}(l)},
$$

where $l$ is the least common multiple of $M_{1}, M_{2}, \cdots, M_{r}$.

It follows from this definition that if

$$
\psi_{(p)}(x)=1+a_{1} x+a_{2} x^{2}+\cdots,
$$


then

$$
\begin{aligned}
f_{(p)}\left(x_{1}, x_{2}, \cdots, x_{r}\right)= & 1+a_{1}\left\{\sum x_{1}-\sum x_{1} x_{2}+\sum x_{1} x_{2} x_{3}-\cdots\right\} \\
& +a_{2}\left\{\sum x_{1}^{2}-\sum x_{1}^{2} x_{2}^{2}+\sum x_{1}^{2} x_{2}^{2} x_{3}^{2}-\cdots\right\}+\cdots \\
= & \sum_{i} \psi_{(p)}\left(x_{i}\right)-\sum_{i, j} \psi_{(p)}\left(x_{i} x_{j}\right)+\sum_{i, j, k} \psi_{(p)}\left(x_{i} x_{j} x_{k}\right)-\cdots
\end{aligned}
$$

We may denote $f$ by the symbol semiprinc $\psi$, or if we wish to put the number of arguments in evidence, by semiprinc $r \psi$. It will be noticed that the derivate of semiprinc $r \psi$ with respect to $s$ of its arguments is simply semiprinc ses $_{r \rightarrow} \psi$.

An analogous definition holds for the case of a matrix-set of arguments. $f\left(\left|M_{i 1}, M_{i 2}, \cdots, M_{i r}\right|\right)(i=1,2, \cdots, s)$ is called the semiprincipal function of $r \times s$ arguments, equivalent to $\psi\left(M_{1}, M_{2}, \cdots, M_{8}\right)$, if, for any prime $p$, $f\left(\left|p^{t_{i 1}}, p^{t_{i 2}}, \cdots, p^{t_{i r}}\right|\right)$ vanishes unless all the non-zero numbers among $t_{i 1}, t_{i 2}, \cdots, t_{i r}$ are equal to each other $(i=1,2, \cdots, s)$, and is equal to $(-1)^{\sigma_{1}+\sigma_{2}+\cdots+\sigma_{\theta}+\delta} \psi\left(p^{\tau_{1}}, p^{\tau_{2}}, \cdots, p^{\tau_{s}}\right)$, if all but $\sigma_{i}$ of the exponents $t_{i 1}, t_{i 2}, \cdots$, $t_{i r}$ are zero, and these equal to $\tau_{i}(i=1,2, \cdots, s)$. As an alternative definition, the semiprincipal function of $r \times s$ arguments $M_{i j}$, equivalent to $\psi\left(M_{1}\right.$, $\left.M_{2}, \cdots, M_{s}\right)$, vanishes whenever the g.c.d. $g_{i j k}$ of $M_{i j}, M_{i k}$ is not relatively prime to both $M_{i j} / g_{i j k}$ and $M_{i k} / g_{i j k}(j, k=1,2, \cdots, r ; i=1,2, \cdots, s)$, and is otherwise equal to

$$
\frac{\psi\left(l_{1}, l_{2}, \cdots, l_{s}\right) \prod_{i j} E_{-1}\left(M_{i j}\right)}{E_{-1}\left(l_{1}\right) E_{-1}\left(l_{2}\right) \cdots E_{-1}\left(l_{s}\right)},
$$

where $l_{i}$ is the least common multiple of $M_{i 1}, M_{i 2}, \cdots, M_{i r}$.

ThEOREM XXXII. The necessary and sufficient condition that $f\left(M_{1}\right.$, $\left.M_{2}, \cdots, M_{r}\right)$ be a function $\psi(l)$ of the l.c.m. lof $M_{1}, M_{2}, \cdots, M_{r}$, is that $f$ be the integral of the semiprincipal function of $r$ arguments equivalent to $\left(\psi \cdot E^{-1}\right)(M)$.

The necessary and sufficient condition that $f\left(\left|M_{i 1}, M_{i 2}, \cdots, M_{i r}\right|\right)(i=1$, $2, \cdots, s)$ be a function $\psi\left(l_{1}, l_{2}, \cdots, l_{s}\right)$ of the l.c.m.'s $l_{i}$ of $M_{i 1}, M_{i 2}, \cdots, M_{i r}$, is that $f$ be the integral of the semiprincipal function of $r \times s$ arguments, equivalent to $\left(\psi \cdot E^{-1}\right)\left(M_{1}, M_{2}, \cdots, M_{8}\right)$.

The sufficiency of the conditions may be easily proved for each case. To prove that the condition is necessary, let

$$
\begin{aligned}
\psi_{(p)}(x) & =\sum A(m) x^{m} ; \\
f_{(p)}\left(x_{1}, x_{2}, \cdots, x_{r}\right) & =\sum a\left(m_{1}, m_{2}, \cdots, m_{r}\right) x_{1}^{m_{1}} x_{2}^{m_{2}} \cdots x_{r}^{m_{r}}, \\
\left(f \cdot E^{-1}\right)_{(p)}\left(x_{1}, x_{2}, \cdots, x_{r}\right) & =\sum b\left(m_{1}, m_{2}, \cdots, m_{r}\right) x_{1}^{m_{1}} x_{2}^{m_{2}} \cdots x_{r}^{{ }^{m_{r}}},
\end{aligned}
$$


where

$b\left(m_{1}, m_{2}, \cdots, m_{r}\right)=a\left(m_{1}, m_{2}, \cdots, m_{r}\right)$

$-\sum a\left(m_{1}-1, m_{2}, \cdots, m_{r}\right)+\sum a\left(m_{1}-1, m_{2}-1, m_{3}, \cdots, m_{r}\right)-$ etc.

Since $f\left(M_{1}, M_{2}, \cdots, M_{r}\right)$ is the function $\psi$ of the l.c.m. of $M_{1}, M_{2}, \cdots, M_{r}$, $a\left(m_{1}, m_{2}, \cdots, m_{r}\right)=A(m)$, where $m$ is the greatest of the suffixes $m_{1}$, $m_{2}, \cdots, m_{r}$. As in the proof of Theorem XXXI, let

$$
m_{1}=m_{2}=\cdots=m_{i}=m ; m_{i+1}, m_{i+2}, \cdots, m_{r}<m .
$$

Also, let $t$ of the $r-i$ numbers $m_{i+1}, m_{i+2}, \cdots, m_{r}$ be equal to zero. Grouping the terms in the expression for $b\left(m_{1}, m_{2}, \cdots, m_{r}\right)$ in such a way, that in each group the values of the first $i$ suffixes remain the same, and summing each group, we obtain

$$
\begin{aligned}
b\left(m_{1}, m_{2}, \cdots,\right. & \left.m_{r}\right) \\
= & (1-1)^{r-i-t}\left\{A(m)-A(m)\left(\begin{array}{l}
i \\
1
\end{array}\right)+A(m)\left(\begin{array}{l}
i \\
2
\end{array}\right)-\cdots\right. \\
& \left.+(-1)^{i-1}\left(\begin{array}{c}
i \\
i-1
\end{array}\right) A_{m}+(-1)^{i} A(m-1)\right\} \\
= & (-1)^{i+1}(1-1)^{r-i-t}\{A(m)-A(m-1)\} .
\end{aligned}
$$

Thus $b\left(m_{1}, m_{2}, \cdots, m_{r}\right)$ vanishes unless all the non-zero $m$ 's are equal, and is equal to $(-1)^{i+1}(A(m)-A(m-1))$, if $r-i$ of the $m$ 's vanish, and the remaining $i$ are all equal to $m$. This proves that $f \cdot E^{-1}$ is the semiprincipal function of $r$ arguments, equivalent to $\left(\psi \cdot E^{-1}\right)(M)$.

An exactly similar proof holds for the case of a matrix-set of arguments.

Example 12. The semiprincipal function of two arguments equivalent to an enumerative totient.

Let $\phi=L \cdot E^{-1}$ be the enumerative totient, and let $T$ denote the totient

$$
\phi^{-1} \times L=L \times\left(L^{-1} \cdot E\right)=L \cdot(L \times L)^{-1} .
$$

Also, let the functional symbol $\phi \phi$ denote the function $\phi(M) \phi(N)$ of two arguments. If

then

$$
\begin{aligned}
\phi_{(p)}(x) & =\frac{1-x}{1-a x}, \\
L_{(p)}(x) & =\frac{1}{1-a x}
\end{aligned}
$$

$$
T_{(p)}(x)=\left\{L \cdot(L \times L)^{-1}\right\}_{(p)}(x)=\frac{1-a^{2} x}{1-a x} .
$$


Now

$$
\begin{aligned}
\left\{\operatorname{semiprinc}_{2}(\phi)\right\}_{(p)}(x, y) & =\frac{1-x}{1-a x}+\frac{1-y}{1-a y}-\frac{1-x y}{1-a x y} \\
& =\frac{\left(1-a^{2} x y\right)(1-x)(1-y)}{(1-a x)(1-a y)(1-a x y)}
\end{aligned}
$$

Hence,

$$
\text { semiprinc } \phi=\phi \phi \cdot \operatorname{princ} T \text {. }
$$

As a consequence of this result, it follows that, if $l$ is the l.c.m. of $M, N$, then

$$
\begin{aligned}
L(l) & =E \cdot \text { semiprinc }\left(L \cdot E^{-1}\right) \\
& =E \cdot \phi \phi \cdot \operatorname{princ}\left(L \cdot(L \times L)^{-1}\right) \\
& =L L \cdot \operatorname{princ}\left(L \cdot(L \times L)^{-1}\right),
\end{aligned}
$$

or

$$
L(l) \cdot \operatorname{princ}(L \times L)=L L \cdot \operatorname{princ} L .
$$

In particular, it also follows that

$$
\left\{I_{a}(l)\right\}_{(p)}(\dot{x}, y)=\frac{1-p^{2 a} x y}{\left(1-p^{a} x\right)\left(1-p^{a} y\right)\left(1-p^{a} x y\right)} .
$$

We add two illustrative results. On putting $L=I_{a}$, it follows, from the expression for the semiprincipal function, that

$$
I_{a}(l) \text { princ } I_{2 a}=I_{a} \cdot \operatorname{princ} I_{a}
$$

Composing both sides of this with $E$, we obtain

$$
I_{a}(l) \cdot E \cdot \operatorname{princ}\left(I_{2 a} \cdot E \cdot E^{-1}\right)=I_{a} \cdot E \cdot \operatorname{princ}\left(I_{a} \cdot E \cdot E^{-1}\right)
$$

or

$$
\sum\left\{l\left(\frac{M}{\delta_{1}}, \frac{N}{\delta_{2}}\right)\right\}^{a} \sigma_{2 a}\left(g\left(\delta_{1}, \delta_{2}\right)\right)=\sum\left(\frac{M N}{\delta_{1} \delta_{2}}\right)^{a} \sigma_{a}\left(g\left(\delta_{1}, \delta_{2}\right)\right) .
$$

Here, the summation extends over all divisors $\delta_{1}$ of $M$, and $\delta_{2}$ of $N$, and $l$ and $g$ denote functions, whose values are respectively the l.c.m. and the g.c.d. of their arguments.

Composing both sides of (A) with $E \cdot$ princ $E^{-1}$, we have 
$E \cdot I_{a}(l) \cdot \operatorname{princ} \phi_{2 a}=I_{a} \cdot E \cdot \operatorname{princ} \phi_{a}$,

where the $\phi$ 's are Jordan functions; or

$$
\sum\left\{l\left(\frac{M}{\delta_{1}}, \frac{N}{\delta_{2}}\right)\right\}^{a}\left\{g\left(\delta_{1}, \delta_{2}\right)\right\}^{2 a}=\sum\left(\frac{M N}{\delta_{1} \delta_{2}}\right)^{a}\left\{g\left(\delta_{1}, \delta_{2}\right)\right\}^{a} .
$$

Section V. Compounding of Functions of SEveral arguments

1. The formula for the compound. We define the compound $f \oplus \phi$ of two functions $f\left(M_{1}, M_{2}, \cdots, M_{r}\right), \phi\left(M_{1}, M_{2}, \cdots, M_{r}\right)$, by

$$
\{f \oplus \phi\}\left(M_{1}, M_{2}, \cdots, M_{r}\right)=\sum f\left(\delta_{1}, \delta_{2}, \cdots, \delta_{r}\right) \phi\left(\frac{M_{1}}{\delta_{1}}, \frac{M_{2}}{\delta_{2}}, \ldots, \frac{M_{r}}{\delta_{r}}\right)
$$

summed for all block-factors $\delta_{i}$ of $M_{i}(i=1,2, \cdots, r)$.

It follows from this definition that the compounding operation performed on several functions is both associative and commutative; also, if

$$
\begin{aligned}
& f_{(p)}\left(x_{1}, x_{2}, \cdots, x_{r}\right)=\sum a\left(m_{1}, m_{2}, \cdots, m_{r}\right) x_{1}^{m_{1}} x_{2}^{m_{2}} \cdots x_{r}^{m_{r}}, \\
& \phi_{(p)}\left(x_{1}, x_{2}, \cdots, x_{r}\right)=\sum b\left(m_{1}, m_{2}, \cdots, m_{r}\right) x_{1}^{m_{1}} x_{2}^{m_{2}} \cdots x_{r}^{m_{r}},
\end{aligned}
$$

then

$$
(f \oplus \phi)_{(p)}\left(x_{1}, x_{2}, \cdots, x_{r}\right)=\sum c\left(m_{1}, m_{2}, \cdots, m_{r}\right) x_{1}^{m_{1}} x_{2}^{m_{2}} \cdots x_{r}^{m_{r}},
$$

where

$$
\begin{gathered}
c\left(m_{1}, m_{2}, \cdots, m_{r}\right)=a\left(m_{1}, m_{2}, \cdots, m_{r}\right)+\sum a\left(0, m_{2}, \cdots, m_{r}\right) b\left(m_{1}, 0,0, \cdots, 0\right) \\
+\sum a\left(0,0, m_{3}, \cdots, m_{r}\right) b\left(m_{1}, m_{2}, 0, \cdots, 0\right)+\text { etc. }
\end{gathered}
$$

Here on the right, the non-zero suffixes among $m_{1}, m_{2}, \cdots, m_{r}$ are partitioned in every possible way between $a$ and $b$, so that the expression for $c\left(m_{1}, m_{2}, \cdots, m_{r}\right)$ contains $2^{i}$ terms, when $r=i$ of $m_{1}, m_{2}, \cdots, m_{r}$ are zero.

The expression for $c\left(m_{1}, m_{2}, \cdots, m_{r}\right)$ shows that

THEOREM XXXIII. The derivate of the compound of any number of functions is the same as the compound of the corresponding derivates of the functions.

Another expression may be given to the compound of $f$ and $\phi$. Write

$$
\begin{aligned}
\psi_{0}\left(x_{1}, x_{2}, \cdots, x_{r}\right)= & f_{(p)}\left(x_{1}, x_{2}, \cdots, x_{r}\right) \\
& +\sum f_{(p)}\left(0, x_{2}, \cdots, x_{r}\right) \phi_{(p)}\left(x_{1}, 0, \cdots, 0\right) \\
& +\sum f_{(p)}\left(0,0, x_{3}, \cdots, x_{r}\right) \phi_{(p)}\left(x_{1}, x_{2}, 0, \cdots, 0\right)+\text { etc., }
\end{aligned}
$$




$$
\begin{aligned}
\psi_{0}\left(x_{1}, x_{2}, \cdots, x_{i}\right)= & f(p)\left(x_{1}, x_{2}, \cdots, x_{i}, 0, \cdots, 0\right) \\
& +\sum f_{(p)}\left(0, x_{2}, \cdots, x_{i}, 0, \cdots, 0\right) \phi_{(p)}\left(x_{1}, 0, \cdots, 0\right) \\
& \quad+\text { etc. }, \\
\psi_{i}\left(x_{1}, x_{2}, \cdots, x_{r}\right)= & \sum \psi_{0}\left(x_{1}, x_{2}, \cdots, x_{i}\right),
\end{aligned}
$$

summed for every set of $i$ of the variables.

Then

$$
(f \oplus \phi)_{(p)}\left(x_{1}, x_{2}, \cdots, x_{r}\right)=\psi_{0}-\psi_{1}+\psi_{2}-\psi_{3}+\cdots .
$$

For the coefficient of $x_{1}^{m_{1}} x_{2}^{m_{2}} \cdots x_{k}^{m_{k}}\left(m_{1}, m_{2}, \cdots, m_{k}>0\right)$ on the left is

$$
\begin{aligned}
C_{k}=a\left(m_{1}, \cdots,\right. & \left.m_{k}, 0, \cdots, 0\right) \\
& +\sum a\left(0, m_{2}, \cdots, m_{k}, 0, \cdots, 0\right) b\left(m_{1}, 0, \cdots, 0\right)+\sum \text { etc. }
\end{aligned}
$$

while its coefficient on the right is seen to be

$$
c_{k}\left(2^{r-k}-\left(\begin{array}{c}
r-k \\
1
\end{array}\right) 2^{r-k-1}+\cdots\right)=(2-1)^{r-k} c_{k}=c_{k} .
$$

This formula for the generating series of the compound is more complicated than the corresponding formula for functions of one argument. But it is important to notice that the two formulas are of the same type, if either $f$ or $\phi$ is a cardinal function. For, if $f$ is a cardinal function, then every coefficient $a$ with a zero suffix vanishes, and the expression for $c$ becomes simply

$$
c\left(m_{1}, m_{2}, \cdots, m_{r}\right)=a\left(m_{1}, m_{2}, \cdots, m_{r}\right)+b\left(m_{1}, m_{2}, \cdots, m_{r}\right) .
$$

The generating series of the compound of functions, all but one of which are cardinal functions, is therefore obtained by adding the generating series of all the functions, and replacing the constant term by unity.

It follows that, while the distributive and quasidistributive properties of the compounding operation (Theorems XIII and XIV) do not hold in general for functions of several arguments, the former continues to hold in the same form, when all but one of the functions compounded are cardinal functions, and the latter, when in addition, the function which enters into composition with the compound is also a cardinal function. Thus if $f$ is a cardinal function, and all but one of $f_{1}, f_{2}, \cdots, f_{k}$ are cardinal functions, then

$$
f \cdot\left(f_{1} \oplus f_{2} \oplus \cdots \oplus f_{k}\right)=\left(f \cdot f_{1}\right) \oplus\left(f \cdot f_{2}\right) \oplus \cdots \oplus\left(f \cdot f_{k}\right) \oplus\left(E_{1-k} \times f\right),
$$

where $E_{1-k}$ is the elementary function of $r$ arguments, which takes the value $1-k$ when the arguments are powers of the same prime. We notice that $E\left(M_{1}, M_{2}, \cdots, M_{r}\right)$ compounded with itself $k-1$ times does not give $E_{k}\left(M_{1}, M_{2}, \cdots, M_{r}\right)$ but $E_{k}\left(M_{1}\right) E_{k}\left(M_{2}\right) \cdots E_{k}\left(M_{r}\right)$.

From the fact that the derivate of the compound is the compound of the 
derivates (Theorem XXXIII), it readily follows that theorems like XXVIII and XXIX should hold with respect to compounding also; namely

THEOREM XXXIV. If two functions of $r$ arguments have identical derivates, each is the compound of the other with a cardinal function.

If two functions of a matrix-set of arguments have identical column-derivates each is the compound of the other with a cardinal function of the matrix-set.

2. The conjugate function. We define the conjugate function by

$$
\operatorname{conj} f \oplus f=E_{0} \text {. }
$$

We shall determine the conjugate of a given function, and thereby show that it exists and is unique. Let

$$
\begin{aligned}
f_{(p)}\left(x_{1}, x_{2}, \cdots, x_{r}\right) & =\sum a\left(m_{1}, m_{2}, \cdots, m_{r}\right) x_{1}^{m_{1}} x_{2}^{m_{2}} \cdots x_{r}^{m_{r}} ; \\
(\operatorname{conj} f)_{(p)}\left(x_{1}, x_{2}, \cdots, x_{r}\right) & =\sum b\left(m_{1}, m_{2}, \cdots, m_{r}\right) x_{1}^{m_{1}} x_{2}^{m_{2}} \cdots x_{r}^{m_{r}} .
\end{aligned}
$$

Since the derivate of a compound is the compound of the corresponding derivates, it follows from the definition of the conjugate function that any derivate of $\operatorname{conj} f$ is the conjugate of the corresponding derivate of $f$. Hence from the formula for the conjugate of a function of one argument (II §6), it follows that

$$
b\left(m_{1}, 0,0, \cdots, 0\right)=-a\left(m_{1}, 0, \cdots, 0\right) \quad\left(m_{1} \neq 0\right) .
$$

Similarly, by taking the derivate of conj $f \oplus f$ with respect to $r-2$ of the arguments, we have

$$
\begin{aligned}
& b\left(m_{1}, m_{2}, 0, \cdots, 0\right)+b\left(0, m_{2}, 0, \cdots, 0\right) a\left(m_{1}, 0, \cdots, 0\right) \\
& +b\left(m_{1}, 0, \cdots, 0\right) a\left(0, m_{2}, 0, \cdots, 0\right)+a\left(m_{1}, m_{2}, 0, \cdots, 0\right)=0 \quad\left(m_{1}, m_{2} \neq 0\right) .
\end{aligned}
$$

Therefore, using the previous result,

$$
\begin{aligned}
b\left(m_{1}, m_{2}, 0, \cdots, 0\right)=-a\left(m_{1}, m_{2}, 0, \cdots, 0\right) \\
+2 ! a\left(m_{1}, 0, \cdots, 0\right) a\left(0, m_{2}, 0, \cdots, 0\right) .
\end{aligned}
$$

This process might be continued. We shall prove by induction that, generally,

$$
\begin{aligned}
& \text { (A) } b\left(m_{1}, m_{2}, \cdots, m_{r}\right) \\
& \quad=\sum_{i}(-1)^{i} i ! \sum a\left(m_{1}, m_{2}, 0, \cdots, 0\right) a\left(0,0, m_{3}, m_{4}, m_{5}, 0, \cdots, 0\right) a(\cdots),
\end{aligned}
$$

where, on the right, the suffixes of the $a$ 's constitute a partition of the nonvanishing $m$ 's into $i$ parts, and the second summation is for all such distinct partitions.

Assume this formula to be true for conjugates of functions of $r-1$ arguments. Then by taking derivates of both sides of the relation 


$$
\operatorname{conj} f \oplus f=E_{0},
$$

it follows that the formula is also true for functions of less than $r-1$ arguments. To establish its truth for the conjugate of a function $f$ of $r$ arguments, we observe that it follows from the definition of the conjugate that

$$
\text { (B) } \begin{aligned}
0=b\left(m_{1}, m_{2}, \cdots, m_{r}\right)+\sum b\left(0, m_{2}, \cdots, m_{r}\right) a\left(m_{1}, 0, \cdots, 0\right) \\
+\cdots+a\left(m_{1}, m_{2}, \cdots, m_{r}\right) .
\end{aligned}
$$

If any of the $m$ 's is zero, then the expression for $b$ follows from the proved truth of formula (A) for functions of less than $r-1$ arguments. We may. therefore take all the $m$ 's to be different from zero. Substituting on the right of (B) for b's with zero suffixes, from formula (A), it becomes evident that the expression for $b\left(m_{1}, m_{2}, \cdots, m_{r}\right)$ is a sum of products of $a$ 's, whose non-zero suffixes constitute a partition of $m_{1}, m_{2}, \cdots, m_{r}$, multiplied by certain numerical coefficients. To find the numerical coefficients of any $a$ product corresponding to an $i$-part partition, say $a\left(m_{1}, m_{2}, 0, \cdots, 0\right) a(0,0$, $\left.m_{3}, m_{4}, m_{5}, 0, \cdots, 0\right) \cdots$, we observe that this product could have come from exactly $i$ terms on the right of (B), namely

$$
\begin{gathered}
a\left(m_{1}, m_{2}, 0, \cdots, 0\right) b\left(0,0, m_{3}, \cdots, m_{r}\right), \\
a\left(0,0, m_{3}, m_{4}, m_{5}, 0, \cdots, 0\right) b\left(m_{1}, m_{2}, 0,0,0, m_{6}, \cdots, m_{r}\right),
\end{gathered}
$$

Substituting for the $b$ 's from (A), it follows that the product in question occurs on the right of $(\mathrm{B})$ with the coefficient $i \cdot(-1)^{i-1}(i-1)$ !. Hence it occurs in the expression for $b\left(m_{1}, m_{2}, \cdots, m_{r}\right)$ with the coefficient $(-1) i i$, proving the truth of (A) for functions of $r$ arguments. Since (A) has been proved to be true for functions of 2 arguments, the induction is complete.

In particular, for the conjugate of a cardinal function $f$, we have the result that if

then

$$
\begin{array}{r}
f_{(p)}\left(x_{1}, x_{2}, \cdots, x_{r}\right)=1+\sum a\left(m_{1}, m_{2}, \cdots, m_{r}\right) x_{1}^{m_{1}} x_{2}^{m_{2}} \cdots x_{r}^{m_{r}} \\
\left(m_{1}, m_{2}, \cdots, m_{r}=1,2, \cdots\right),
\end{array}
$$

$(\operatorname{conj} f)_{(p)}\left(x_{1}, x_{2}, \cdots, x_{r}\right)=1-\sum a\left(m_{1}, m_{2}, \cdots, m_{r}\right) x_{1}^{m_{1}} x_{2}^{m_{2}} \cdots x_{r}^{m_{r}}$.

Thus the formula which gives the conjugate of a cardinal function is analogous to the formula for conjugates of functions of a single argument.

3. The compounding of functions of a matrix-set. The two theories of the compounding process, which have been stated for functions of one argument and of several arguments, respectively, have yielded formally different results, even though they have the same arithmetical basis. The reason 
for the difference lies in the fact that, while in $(f \oplus \phi)(M)$ a term of the type $f(1) \phi(M)$ occurs only once, there are in $(f \oplus \phi)\left(M_{1}, M_{2}, \cdots, M_{r}\right)\left(2^{r}-1\right)$ terms of the type $f\left(\delta_{1}, \delta_{2}, \cdots, \delta_{r}\right) \times \phi\left(M_{1} / \delta_{1}, M_{2} / \delta_{2}, \cdots, M_{r} / \delta_{r}\right)$, in which one or more of the block-factors $\delta$ are equal to unity. It is of course open to us to develop a theory of the compound of functions of several arguments, which is formally similar to that for a single argument; we could to this by using the definition

$$
(f \oplus \phi)\left(M_{1}, M_{2}, \cdots, M_{r}\right)=\sum f\left(\delta_{1}, \delta_{2}, \cdots, \delta_{r}\right) \phi\left(\frac{M_{1}}{\delta_{1}}, \frac{M_{2}}{\delta_{2}}, \ldots, \frac{M_{r}}{\delta_{r}}\right),
$$

where the summation is for all sets $\delta_{1}, \delta_{2}, \cdots, \delta_{r}$ of block-factors, which have the property that no factor of any $\delta$ is prime to any other $\delta$ (in other words, that the distinct prime factors of every $\delta$ are the same), with a similar property for the set $M_{i} / \delta_{i}$. It is clear that the compound defined thus is a multiplicative function, and indeed (as will be seen below) a cardinal function, of $M_{1}, M_{2}, \cdots, M_{r}$. We shall describe this definition of the compound, by saying that, in it, the argument-group $\left(M_{1}, M_{2}, \cdots, M_{r}\right)$ behaves like a single argument. The possibility of this alternative definition suggests a more general view of the compounding operation, which embraces the two theories as aspects of itself.

Let a given set of arguments be divided into mutually exclusive groups in any manner. The most general definition of the compound of two functions of these arguments is then that the compound is formed in the usual way, with the modification that each group of arguments behaves like a single argument, in the sense defined above.

This generalized view of the compound will always be understood, whenever there is any indication of grouping in the arguments. For example, when we talk of the compound of two functions of a matrix set of arguments, it will be understood, by convention, that the arguments in each column of the matrix have been grouped together, so as to behave like a single argument in the above sense.

It is easy to see that the generating series, to any base, of the compound of two functions $f, \phi$ of the matrix $\left|M_{i 1}, M_{i 2}, \cdots, M_{i r}\right|(i=1,2, \cdots, s)$, need not be worked out ab initio, but may be seen at a glance, by a proper interpretation of the formula $(\mathrm{V} \S 1)$ for the case of $r$ arguments, viz.

$$
\begin{aligned}
c\left(m_{1}, m_{2}, \cdots, m_{r}\right)=a(0,0, \cdots, & 0) b\left(m_{1}, \cdots, m_{r}\right) \\
& +\sum a\left(m_{1}, 0, \cdots, 0\right) b\left(0, m_{2}, \cdots, m_{r}\right)+\text { etc. }
\end{aligned}
$$

To do this, we consider that $\sum a\left(m_{1}, m_{2}, \cdots, m_{r}\right) x^{m_{1}} \cdots x_{r}^{m_{r}}$ and $\sum b\left(m_{1}, \cdots\right.$, $\left.m_{r}\right) x_{f^{m_{1}}} \cdots x_{r}^{m_{r}}$ still represent the generating series of the functions $f, \phi$ of the 
matrix set, on the understanding that each $x_{j}^{m_{i}}$ stands for $x_{1 j}^{m_{1 j}} x_{2 j}^{m_{2 j}} \ldots x_{s j}^{m_{s j} j}$, and each $m_{j}$ in the coefficients stands for the group of indices $m_{1 j}, m_{2 j}, \cdots, m_{s j}$. If, further, we interpret the occurrence of a zero in place of $m_{j}$ to mean $m_{1 j}=m_{2 j}=\cdots=m_{s i}=0$, then the above formula determines the generating series of the compound. For, the expression for the compound of two functions $f, \phi$, when regarded as functions of the matrix $\left|M_{i j}\right|$, differs from the corresponding expression when they are regarded as functions of the $r \times s$ arguments $M_{i j}$, in the suppression of all those terms $f\left(\delta_{i j}\right) \phi\left(M_{i j} / \delta_{i j}\right)$, for which the elements in any column of $\left|\delta_{i j}\right|$ or $\left|M_{i j} / \delta_{i j}\right|$ do not all involve the same prime factors; hence it follows that the expression for the general coefficient $c\left(m_{i j}\right)$ of the generating series of the compound in the former case, differs from its expression in the latter in the suppression of those terms $a\left(m_{i j}\right), b\left(m_{i j}\right)$, in which some only of the elements of a column of $\left|m_{i j}\right|$ are zero.

In particular, it follows from the expression for $c\left(m_{i j}\right)$, that $c\left(m_{i j}\right)=0$, when one element from each column of $\left|m_{i j}\right|$ is zero, without all the $m$ 's vanishing simultaneously.

Definition. A function $F$ of the matrix $\left|M_{i 1} M_{i 2} \cdots M_{i r}\right|(i=1,2, \cdots, s)$ will be called a transcardinal function, if its derivate with respect to every set of $r$ arguments, chosen one from each column, is the function $E_{0}$.

The following points may be noted in connection with this definition and the correlated definition of the cardinal function of a matrix:

(1) A transcardinal function of $\left|M_{i j}\right|$ is necessarily a cardinal function of the transposed matrix $\left|M_{i i}\right|$, but the converse is not true.

(2) A transcardinal function of the "long matrix" $\left|M_{1}, M_{2}, \cdots, M_{r}\right|$ is any arbitrary function of $M_{1}, M_{2}, \cdots, M_{r}$; a transcardinal function of the "deep matrix"

$$
\left|M_{i}\right|=\left|\begin{array}{c}
M_{1} \\
M_{2} \\
\vdots
\end{array}\right|
$$

is a cardinal function of $M_{1}, M_{2}, \cdots, M_{r}$.

(3) A cardinal function of the long matrix $\left|M_{1}, \cdots\right|$ is a cardinal function of $M_{1}, M_{2}, \cdots, M_{r}$, a cardinal function of the deep matrix $\left|M_{i}\right|$ is an arbitrary function of $M_{1}, M_{2}, \cdots, M_{r}$.

Theorem XXIII(a). The compound of any two functions of a matrix $\left|M_{i j}\right|$ is a transcardinal function of $\left|M_{i j}\right|$.

This may be seen directly from the definition of the compound, or thus. 
By definition, a transcardinal function vanishes when one argument in each of the columns of $\left|M_{i j}\right|$ is equal to 1 , unless all the other arguments are also equal to 1 . Hence, the coefficient $c\left(m_{i j}\right)$ of the general term of its generating series vanishes when one element from each column of $\left|m_{i j}\right|$ is zero, unless all the remaining $m$ 's are also zero. But this property was noted above to hold for the compound of two functions of a matrix, hence the theorem.

From this theorem it follows that if $f$ is a function of the matrix which is not transcardinal, $f \oplus E_{0}$ cannot be equal to $f$; as a matter of fact we see directly that $f \oplus E_{0}$ is that transcardinal function of the matrix, which is equal to $f$ for all sets of values of the arguments, except those for which it vanishes in virtue of being a transcardinal function. We shall write

$$
f \oplus E_{0}=\operatorname{tcrd} f
$$

and call tcrd $f$ the transcardinal part of $f$.

THEOREM XXIII(b). The compound of two functions $f, \phi$ of a matrix depends only on the transcardinal parts of $f$ and $\phi$.

For, if $f \oplus \phi=\psi$, then $\psi$ is transcardinal by the previous theorem, so that tcrd $\psi=\psi$. Hence

$$
\begin{aligned}
f \oplus \phi=\psi=\operatorname{tcrd} \psi & =\psi \oplus E_{0} \\
& =f \oplus \phi \oplus E_{0}=f \oplus \operatorname{tcrd} \phi .
\end{aligned}
$$

The conjugate of the function $f$ of the matrix $\left|M_{i j}\right|$ is defined to be another function $\operatorname{conj} f$ of the same matrix, such that

$$
f \oplus \operatorname{conj} f=E_{0} .
$$

Since the formula for the generating series of the conjugate function is simply a consequence of that for a compound, it follows that we can deduce the generating series of $\operatorname{conj} f$ from that of $f$, from the result $(\mathrm{V}$ \&)

$$
b\left(m_{1}, m_{2}, \cdots, m_{r}\right)=\sum(-1)^{i} i ! \sum a\left(m_{1}, m_{2}, 0, \cdots\right) a\left(0,0, m_{3}, \cdots\right) \cdots,
$$

by proceeding in the same manner as was done in the case of the compound. In particular it follows that the conjugate of a cardinal function $f$ of the matrix is another cardinal function of the same matrix, whose generating series to any base is obtained from that of $f$, by changing the sign of all the terms except the constant term.

4. Rational functions of several arguments. (Cf. III.) The function $f\left(M_{1}, M_{2}, \cdots, M_{r}\right)$ is said to be a rational function, if 


$$
f_{(p)}\left(x_{1}, x_{2}, \cdots, x_{r}\right)=\frac{F_{(p)}\left(x_{1}, x_{2}, \cdots, x_{r}\right)}{\phi_{(p)}\left(x_{1}, x_{2}, \cdots, x_{r}\right)},
$$

where $F_{(p)}$ and $\phi_{(p)}$ are polynomials for every $p$, whose degrees $\rho_{i}, \sigma_{i}$ in $x_{i}$ have finite upper bounds as $p \rightarrow \infty(i=1,2, \cdots, r)$; the functions whose generating series are $\phi_{(p)}$ and $F_{(p)}$ are, respectively, the integral and inverse component of $f$.

Since polynomials in more than one variable are not necessarily factorable, a rational function can not in general be expressed as a composite of functions of lower degrees.

The composite of rational functions $f_{1}, f_{2}$ is clearly a rational function, whose integral and inverse components are, respectively, the composites of the integral and inverse components of $f_{1}, f_{2}$. Thus composition is a rational and integral process. It is easy to show that the compound of $f_{1}, f_{2}$ is a rational function whose integral component is the composite of the integral components of $f_{1}, f_{2}$, and of all products of complementary derivates of $f_{1}, f_{2}$. The product of rational functions is also a rational function. This may be proved by a method similar to the one adopted for functions of a single argument. It could be inferred directly, if Hadamard's multiplication theorem can be extended to power series in several variables; the extension appears however to have been carried out so far only to two variables.*

We have to distinguish between a linear function of $r$ arguments, and a rational integral function of degree 1 ; the former is a product of linear functions of single arguments, and is a particular case of the latter. We define a totient as the composite of a linear function and the inverse of a linear function; thus the totient is, like the linear function, a separable function, being the product of totients of single arguments (a function of $r$ arguments is separable, if it is the product of functions of fewer arguments). Thus a totient of $r$ arguments is not the most general rational function of degree $(1,1)$ in each of the arguments.

Section VI. The identical EQUation of the MUltiplicative function

1. The cardinal functions associated with a function of $r$ arguments. The multiplicative property of $f\left(M_{1}, M_{2}, \cdots, M_{r}\right)$ implies that

$$
f\left(M_{1} N_{1}, M_{2} N_{2}, \cdots, M_{r} N_{r}\right)=f\left(M_{1}, M_{2}, \cdots, M_{r}\right) f\left(N_{1}, N_{2}, \cdots, N_{r}\right),
$$

whenever the products $M_{1} M_{2} \cdots M_{r}, N_{1} N_{2} \cdots N_{r}$ are relatively prime. Now the functions on both sides of this equation are functions of the matrix-

* Cf. U. S. Haslam-Jones, An extension of Hadamard's multiplication theorem, Proceedings of the London Mathematical Society, (2), vol. 27. 
set of $2 \times r$ arguments $\left|M_{i}, N_{i}\right|(i=1,2, \cdots, r)$, and the equation asserts the equality of the functions, whenever the column-products are relatively prime. Hence it follows from Theorems XXIX, and XXXIV, that $f\left(M_{1} N_{1}, M_{2} N_{2}, \cdots, M_{r} N_{r}\right)$ is

(1) the composite of $f f$ and a cardinal function $C_{1}$ of the matrix $\left|M_{i}, N_{i}\right|$ (where we use $f f$ as the functional symbol for $f\left(M_{1}, M_{2}, \cdots, M_{r}\right) f\left(N_{1}\right.$, $\left.N_{2}, \cdots, N_{r}\right)$ ),

(2) the product of $f f$ and the integral of a cardinal function $C_{2}$ of the same matrix,

(3) and, lastly, the compound of $f f$ and a cardinal function $C_{3}$, of the same matrix.

It will be convenient to refer to $C_{1}, C_{2}, C_{3}$, as the first, second, and third cardinal functions associated with $f\left(M_{1}, M_{2}, \cdots, M_{r}\right)$. Unlike the second and third cardinal functions, the first cardinal function can be expressed in a simple manner in terms of $f$, by means of the processes of the calculus. Namely, when $F$ is a function of a matrix-set of arguments, let us denote by crd $F$ (the cardinal of $F$ ) that cardinal function of the matrix-set, which is equal to $F$, except for those values of the arguments for which it vanishes in virtue of being a cardinal function. It is easy to see that the generating series of $\operatorname{crd} F$ is obtained from the corresponding generating series of $F$, by suppressing all those terms, in which the variables corresponding to a column of the matrix-set are entirely absent. With this notation, it will now be shown that the first cardinal function of $f\left(M_{1}, M_{2}, \cdots, M_{r}\right)$ is the conjugate* of $\operatorname{crd} f^{-1}\left(M_{1} N_{1}, M_{2} N_{2}, \cdots, M_{r} N_{r}\right)$, where $f^{-1}$ represents the inverse function of $r$ arguments, of $f$.

2. The expression for the first cardinal function. The first cardinal function $C_{1}\left(\left|M_{i}, N_{i}\right|\right)$ associated with $f$ is given by

$$
f\left(M_{1} N_{1}, M_{2} N_{2}, \cdots, M_{r} N_{r}\right)=f f \cdot C_{1} .
$$

Now, the inverse of $f f$ is $f^{-1} f^{-1}$ (Theorem $\mathrm{X}$ ). Hence

$$
C_{1}=f\left(M_{1} N_{1}, \cdots, M_{r} N_{r}\right) \cdot f^{-1} f^{-1} .
$$

To show that this is equal to

$$
\text { conj } \operatorname{crd} f^{-1}\left(M_{1} N_{1}, M_{2} N_{2}, \cdots, M_{r} N_{r}\right),
$$

we shall, for convenience of writing, take $r=2$, the method being the same for any value of $r$. Let

* Notice that this is the conjugate of a function of a matrix, and must therefore be understood not in the sense of $\mathrm{V} \S 2$, but in the generalized sense of $\mathrm{V} \S 3$. 


$$
\begin{aligned}
& f_{(p)}\left(x_{1}, x_{2}\right)=\sum a\left(m_{1}, m_{2}\right) x_{1}^{m_{1}} x_{2}^{m_{2}}, \\
& f_{(p)}^{-1}\left(x_{1}, x_{2}\right)=\sum b\left(m_{1}, m_{2}\right) x_{1}^{m_{1}} x_{2}^{m_{2}},
\end{aligned}
$$

so that

$$
\sum b\left(m_{1}-\mu_{1}, m_{2}-\mu_{2}\right) a\left(\mu_{1}, \mu_{2}\right)=1 \text { or } 0,
$$

according as $m_{1}, m_{2}$ are, or are not, simultaneously zero $\left(\mu_{1}=0,1, \cdots, m_{1}\right.$; $\left.\mu_{2}=0,1, \cdots, m_{2}\right)$. Then,

$$
\begin{aligned}
\left\{f\left(M_{1} N_{1}, M_{2} N_{2}\right)\right\}_{(p)}\left(x_{1}, y_{1}, x_{2}, y_{2}\right) & =\sum a\left(m_{1}+n_{1}, m_{2}+n_{2}\right) x_{1}^{m_{1}} y_{1}^{n_{1}} x_{2}^{m_{2}} y_{2}^{n_{2}} ; \\
\left\{f^{-1}\left(M_{1}, M_{2}\right) f^{-1}\left(N_{1}, N_{2}\right)\right\}_{(p)}\left(x_{1}, x_{2}, y_{1}, y_{2}\right) & =\sum b\left(m_{1}, m_{2}\right) b\left(n_{1}, n_{2}\right) x_{1}^{m_{1}} y_{1}^{n_{1}} x_{2}^{m_{2}} y_{2}^{n_{2}} .
\end{aligned}
$$

Hence, if

$$
C_{1(p)}\left(x_{1}, x_{2}, y_{1}, y_{2}\right)=\sum c\left(m_{1}, m_{2}, n_{1}, n_{2}\right) x_{1}^{m_{1}} x_{2}^{m_{2}} y_{1}^{n_{1}} y_{2}^{n_{2}},
$$

we must have

$$
\begin{aligned}
c\left(m_{1}, m_{2}, n_{1}, n_{2}\right) & =\sum a\left(\mu_{1}+\nu_{1}, \mu_{2}+\nu_{2}\right) b\left(m_{1}-\mu_{1}, m_{2}-\mu_{2}\right) b\left(n_{1}-\nu_{1}, n_{2}-\nu_{2}\right) \\
\left(\mu_{1}=0,1, \cdots,\right. & \left.m_{1} ; \mu_{2}=0,1, \cdots, m_{2} ; \nu_{1}=0,1, \cdots, n_{1} ; \nu_{2}=0,1, \cdots, n_{2}\right) \\
& =\sum_{\nu_{1}, \nu_{2}} b\left(n_{1}-\nu_{1}, n_{2}-\nu_{2}\right) \sum_{\mu_{1}, \mu_{2}} a\left(\mu_{1}+\nu_{1} ; \mu_{2}+\nu_{2}\right) b\left(m_{1}-\mu_{1}, m_{2}-\mu_{2}\right) .
\end{aligned}
$$

Since $C_{1}$ is a cardinal function, $c\left(m_{1}, m_{2}, n_{1}, n_{2}\right)$ vanishes when $m_{1}, m_{2}$ (or $\left.n_{1}, n_{2}\right)$ are simultaneously zero. We may therefore suppose that $m_{1}, m_{2}$ are not both zero, so that $m_{1}+\nu_{1}$ and $m_{2}+\nu_{2}$ are not simultaneously zero, for any admissible values of $\nu_{1}, \nu_{2}$. Hence, by (A) above,

$$
\begin{aligned}
& \sum_{\mu_{1}, \mu_{2}} a\left(\mu_{1}+\nu_{1}, \mu_{2}+\nu_{2}\right) b\left(m_{1}-\right.\left.\mu_{1}, m_{2}-\mu_{2}\right) \\
&=-\sum_{k_{1}, k_{2}} a\left(\nu_{1}-k_{1}, \nu_{2}-k_{2}\right) b\left(m_{1}+k_{1}, m_{2}+k_{2}\right) \\
&\left(k_{1}=0,1, \cdots, \nu_{1} ; k_{2}=0,1, \cdots, \nu_{2} ; k_{1} \text { and } k_{2} \text { not both zero }\right) .
\end{aligned}
$$

Therefore,

$$
\begin{array}{r}
c\left(m_{1}, m_{2}, n_{1}, n_{2}\right)= \\
=\sum_{k_{1}, k_{2}}-b\left(m_{1}+k_{1}, m_{2}+k_{2}\right) \sum_{\nu_{1}, \nu_{2}} b\left(n_{1}-\nu_{1}, n_{2}-\nu_{2}\right) a\left(\nu_{1}-k_{1}, \nu_{2}-k_{2}\right) \\
\quad\left(\nu_{1}=k_{1}, k_{1}+1, \cdots, n_{1} ; \nu_{2}=k_{2}, k_{2}+1, \cdots, n_{2}\right) .
\end{array}
$$

It is clear that here the value of the inner sum is zero, unless $n_{1}-k_{1}, n_{2}-k_{2}$ are simultaneously zero, in which case it is 1 . Now $k_{1}=n_{1}, k_{2}=n_{2}$ is a set of values within the range of $k_{1}, k_{2}$, only when $n_{1}, n_{2}$ are not both zero. We see, therefore, that 


$$
\begin{aligned}
c\left(m_{1}, m_{2}, n_{1}, n_{2}\right) & =0, \text { if either } m_{1}, m_{2}, \text { or } n_{1}, n_{2} \text { are both zero, } \\
& =-b\left(m_{1}+n_{1}, m_{2}+n_{2}\right), \text { otherwise. }
\end{aligned}
$$

But the cardinal function, the generating series of which has $-b\left(m_{1}+n_{1}\right.$, $\left.m_{2}+n_{2}\right)$ for the coefficient of $x_{1}^{m_{1}} x_{2}^{m_{2}} y_{1}^{n_{1}} y_{2}^{n_{2}}$, is obviously conj $\operatorname{crd} f^{-1}\left(M_{1} N_{1}\right.$, $\left.M_{2} N_{2}\right)$.* The same method shows that, generally,

$$
c_{1}=\text { conj } \operatorname{crd} f^{-1}\left(M_{1} N_{1}, M_{2} N_{2}, \cdots, M_{r} N_{r}\right) \text {. }
$$

The equation

$$
f\left(M_{1} N_{1}, M_{2} N_{2}, \cdots, M_{r} N_{r}\right)=f f \cdot \operatorname{conj} \operatorname{crd} f^{-1}\left(M_{1} N_{1}, M_{2} N_{2}, \cdots, M_{r} N_{r}\right)
$$

is thus identically satisfied by any multiplicative function $f$, and will be referred to as the identical equation of $f$.

For functions of one argument, the identical equation takes the form

$$
f(u v)=\{f(u) f(v)\} \cdot\left\{\text { conj } \operatorname{crd} f^{-1}(u v)\right\} .
$$

3. The identical equation of a totient. Let $T=L_{1} \cdot L_{2}^{-1}$ be a totientfunction of a single argument, so that its identical equation is

Let

$$
T(M N)=T(M) T(N) \cdot \operatorname{conj} \operatorname{crd} T^{-1}(M N) .
$$

$$
T_{(p)}(x)=\frac{1-\beta x}{1-\alpha x} ; \quad T_{(p)}^{-1}(x)=\frac{1-\alpha x}{1-\beta x} .
$$

Then

Hence

$$
\begin{aligned}
\left\{\operatorname{conj} \operatorname{crd} T^{-1}(M N)\right\}_{(p)}(x, y) & =1-\beta^{2}\left(1-\frac{\alpha}{\beta}\right) x y\left\{\sum \beta^{m+n} x^{m} y^{n}\right\} \\
& =\frac{1-\beta(x+y)+\alpha \beta x y}{(1-\beta x)(1-\beta y)} .
\end{aligned}
$$

conj $\operatorname{crd} T^{-1}(M N)=L_{2}(M) L_{2}(N) \cdot\left\{L_{2}^{-1}(\right.$ l.c.m of $M, N) \times L_{1}^{-1}($ g.c.d of $\left.M, N)\right\}$.

Thus the identical equation for $T$ becomes

$$
\begin{aligned}
T(M N) & \left.=L_{1}(M) L_{1}(N) \cdot{L_{2}}^{-1}(M) L_{2}^{-1}(N) \cdot L_{2}(M) L_{2}(N) \cdot\left\{L_{2}^{-1}(l) \times{L_{1}^{-1}}^{-1} g\right)\right\} \\
& =L_{1}(M) L_{1}(N) \cdot\left\{L_{2}^{-1}(l) \times L_{1}^{-1}(g)\right\} \\
& =L_{1}(M) L_{1}(N) \cdot\left\{L_{2}(l) \times L_{1}(g) \times \mu(M) \mu(N)\right\}
\end{aligned}
$$

where $g, l$ are, respectively, the g.c.d. and the l.c.m. of $M, N$.

For example, the identical equation of the totient $\phi_{r, k}=I_{r} \cdot \lambda_{k}^{-1}$ (which

* Compare V §3. 
reduces to Jordan's function for $k=1$, and for $k=\mathrm{a}$ positive integer, is identical with Schemmel's extension of Euler's function,* when the argument has no prime factors equal to, or less than $k$ ) is

$$
\phi_{r, k}(M N)=\sum\left(\frac{M N}{\delta_{1} \delta_{2}}\right)^{r} \lambda_{k}\left\{l\left(\delta_{1}, \delta_{2}\right)\right\}\left\{g\left(\delta_{1}, \delta_{2}\right)\right\}^{r} \mu\left(\delta_{1}\right) \mu\left(\delta_{2}\right)
$$

or

$$
\frac{\phi_{r, k}(M N)}{(M N)^{r}}=\sum E_{k}\left(\delta_{1} \delta_{2}\right) \cdot \frac{\left\{g\left(\delta_{1}, \delta_{2}\right)\right\}^{r}}{\left(\delta_{1} \delta_{2}\right)^{r}} \mu\left(\delta_{1}\right) \mu\left(\delta_{2}\right) .
$$

If $T$ is an enumerative totient, then $L_{2}=E$, and the identical equation takes the form

$$
T(M N)=L_{1}(M) L_{1}(N) \cdot\left\{L_{1}(g) \mu(M) \mu(N)\right\} .
$$

In particular, for the Jordan function

$$
\frac{\phi_{r}(M N)}{M^{r} N^{r}}=\sum \frac{\left\{g\left(\delta_{1}, \delta_{2}\right)\right\}^{r}}{\left(\delta_{1} \delta_{2}\right)^{r}} \mu\left(\delta_{1}\right) \mu\left(\delta_{2}\right),
$$

a result which may also be derived from (A).

4. The Busche-Ramanujan identity. E. Busche† stated that

$$
\sigma_{a}(M) \sigma_{a}(N)=\sum d^{a} \sigma_{a}\left(\frac{M N}{d^{2}}\right)
$$

summed over the common divisors $d$ of $M$ and $N$; Ramanujan has utilised this result (for the case $a=0$ ), as well as its inverse form: $f$

$$
\sigma_{a}(M N)=\sum \sigma_{a}\left(\frac{M}{d}\right) \sigma_{a}\left(\frac{N}{d}\right) d^{a} \mu(d) .
$$

We shall say generally that a function $f\left(M_{1}, M_{2}, \cdots, M_{r}\right)$ admits $a$ BuscheRamanujan identity, if we have identically

$$
\begin{aligned}
f\left(M_{1} N_{1}, M_{2} N_{2}, \cdots, M_{r} N_{r}\right) \\
=\sum f\left(\frac{M_{1}}{\delta_{1}}, \frac{M_{2}}{\delta_{2}}, \ldots, \frac{M_{r}}{\delta_{r}}\right) f\left(\frac{N_{1}}{\delta_{1}}, \frac{N_{2}}{\delta_{2}}, \ldots, \frac{N_{r}}{\delta_{r}}\right) F\left(\delta_{1}, \delta_{2}, \cdots, \delta_{r}\right),
\end{aligned}
$$

* Dickson, p. 147.

† Dickson, p. 319, Note 147.

† Collected Papers, p. 134. 
summed for common divisors $\delta_{i}$ of $M_{i}, N_{i}(i=1,2, \cdots, r), F$ being some (necessarily multiplicative) function of $r$ arguments.

A relation of this form asserts that $f\left(M_{1} N_{1}, M_{2} N_{2}, \cdots, M_{r} N_{r}\right)$ is the composite of $f f$ and a principal function of the matrix-set $\left|M_{i}, N_{i}\right|(i=1$, $2, \cdots, r)$. Hence a function $f$ admits a Busche-Ramanujan identity, if, and only if, its first cardinal function is a principal function; that is, only if conj $\operatorname{crd} f^{-1}\left(M_{1} N_{1}, M_{2} N_{2}, \cdots, M_{r} N_{r}\right)$, and therefore also $\operatorname{crd} f^{-1}\left(M_{1} N_{1}\right.$, $\left.M_{2} N_{2}, \cdots, M_{r} N_{r}\right)$, becomes a principal function. To find the condition that this may be the case, let

$$
f_{(p)}^{-1}\left(x_{1}, x_{2}, \cdots, x_{r}\right)=\sum b\left(m_{1}, m_{2}, \cdots, m_{r}\right) x_{1}^{m_{1}} x_{2}^{m_{2}} \cdots x_{r}^{m_{r}},
$$

so that

$$
\begin{aligned}
\left\{\operatorname{crd} f^{-1}\left(M_{1} N_{1}, M_{2} N_{2}, \cdots, M_{r} N_{r}\right)\right\}_{(p)}\left(x_{1}, \cdots, x_{r}, y_{1}, \cdots, y_{r}\right) \\
=1+\sum b\left(m_{1}+n_{1}, \cdots, m_{r}+n_{r}\right) x_{1}^{m_{1}} y_{1}^{n_{1}} \cdots x_{r}^{m_{r}} y_{r}^{n_{r}},
\end{aligned}
$$

where, on the right, simultaneous zero values of $m_{1}, m_{2}, \cdots, m_{r}$ and $n_{1}$, $n_{2}, \cdots, n_{r}$ are excepted from the summation. The condition that this may be a principal function is the vanishing of $b\left(m_{1}+n_{1}, m_{2}+n_{2}, \cdots, m_{r}+n_{r}\right)$ when any $m_{i} \neq n_{i}$ (with the understanding that neither all the $m$ 's nor all the $n$ 's vanish together). In other words $b\left(\lambda_{1}, \lambda_{2}, \cdots, \lambda_{r}\right)=0$, whenever the $\lambda$ 's admit a partition $\lambda_{i}=m_{i}+n_{i}(i=1,2, \cdots, r)$, in which at least one $m_{i} \neq n_{i}$, neither the $m$ 's nor the $n$ 's being all zero. Hence

(1) $b\left(\lambda_{1}, \lambda_{2}, \cdots, \lambda_{r}\right)=0$, if any $\lambda>2$. For if $\lambda_{i}=3$ (say), we can write $\lambda_{i}=m_{i}+n_{i} ; m_{i}=1, n_{i}=2 ; m_{i} \neq n_{i} ; m_{i} \neq 0, n_{i} \neq 0$.

(2) $b\left(\lambda_{1}, \lambda_{2}, \cdots, \lambda_{r}\right)=0$, if at least two of the $\lambda$ 's do not vanish. For, if $\lambda_{1} \neq 0, \lambda_{2} \neq 0$, we can write $\lambda_{1}=m_{1}+n_{1} ; \lambda_{2}=m_{2}+n_{2} ; m_{1}=\lambda_{1}, n_{1}=0 ; m_{2}=0$, $n_{2}=\lambda_{2}$.

It results that $b\left(\lambda_{1}, \lambda_{2}, \cdots, \lambda_{r}\right)$ can have only $2 r$ non-vanishing values $b_{i}, c_{i}$, corresponding to $\lambda_{2}=1$ or $2 ; \lambda_{j}=0$ for $j \neq i$. Thus it follows that

$$
\begin{aligned}
f_{(p)}^{-1}\left(x_{1}, x_{2}, \cdots, x_{r}\right)=1 & +b_{1} x_{1}+b_{2} x_{2}+\cdots \\
& +b_{r} x_{r}+c_{1} x_{1}{ }^{2}+c_{2} x_{2}^{2}+\cdots+c_{r} x_{r}^{2} .
\end{aligned}
$$

Therefore

$$
f_{(p)}\left(x_{1}, x_{2}, \cdots, x_{r}\right)=\frac{1}{1+\sum b_{i} x_{i}+}-\overline{\sum c_{i} x_{i}^{2}} .
$$

Hence $f\left(M_{1}, M_{2}, \cdots, M_{r}\right)$ is an integral function of a special type, quadratic in each of its arguments, its special property being that $f^{-1}\left(M_{1}, M_{2}, \cdots, M_{r}\right)$ 
vanishes unless every two of its arguments are relatively prime. ${ }^{*}$ Also, vrhen $f$ is a function of this form,

$$
\begin{array}{r}
\left\{\operatorname{conj} \operatorname{crd} f^{-1}\left(M_{1} N_{1}, M_{2} N_{2}, \cdots, M_{r} N_{r}\right)\right\}_{(p)}\left(x_{1}, \cdots, x_{r}, y_{1}, \cdots, y_{r}\right) \\
=1-\sum c_{i} x_{i} y_{i} .
\end{array}
$$

Now the derivate of $f\left(M_{1}, M_{2}, \cdots, M_{r}\right)$ with respect to the $r-1$ arguments other than $M_{i}$, is evidently an integral quadratic function of $M_{i}$, which we may denote by $L_{i} \cdot L_{i}^{\prime}$; we have

$$
\left(L_{i} \cdot L_{i}^{\prime}\right)_{(p)}^{j}\left(x_{i}\right)=\frac{1}{1+b_{i} x_{i}+c_{i} x_{i}^{2}}(i=1,2, \cdots, r) \text {; }
$$

$$
\left(L_{i} \times L_{i}^{\prime}\right)_{(p)}^{-1}\left(x_{i}\right)=1-c_{i} x_{i} .
$$

It therefore follows that conj $\operatorname{crd} f^{-1}\left(M_{1} N_{1}, \cdots, M_{r} N_{r}\right)$ is a principal function, princ $F$ (say), where

$F\left(M_{1}, M_{2}, \cdots, M_{r}\right)=0$, if two of the arguments have a common factor,

$$
=K_{1}^{-1}\left(M_{1}\right) K_{2}^{-1}\left(M_{2}\right) \cdots K_{r}^{-1}\left(M_{r}\right), \text { otherwise, }
$$

where

$$
K_{i}=L_{i} \times L_{i}^{\prime} \quad(i=1,2, \cdots, r) .
$$

We have thus reached the following theorem:

THEOREM XXXV. The only functions of $r$ arguments which admit a BuscheRamanujan identity are the integral quadratic functions, whose inverses vanish unless every two of the arguments are relatively prime. The identity for a function $f$ of this type has the form

$$
f\left(M_{1} N_{1}, \cdots, M_{r} N_{r}\right)=f f \text { princ } F,
$$

where $F\left(M_{1}, M_{2}, \cdots, M_{r}\right)=0$, if any two of its arguments have a common factor, and is otherwise equal to $K_{1}^{-1}\left(M_{1}\right) K_{2}^{-1}\left(M_{2}\right) \cdots K_{r}^{-1}\left(M_{r}\right) ; K_{i}=L_{i} \times L_{i}^{\prime}$; and $L_{i} \cdot L_{i}^{\prime}\left(M_{i}\right)$ is the derivate of $f$ with respect to the $r-1$ arguments other than $M_{i}$.

In particular, the functions $f$ of one argument, which admit a BuscheRamanujan identity, are the integral quadratic functions and these only. When $f=L_{1} \cdot L_{2}$ the identity has the form

$$
f(M N)=f(M) f(N) \cdot \operatorname{princ}\left(L_{1} \times L_{2}\right)^{-1} .
$$

\footnotetext{
* Functions with this property are, in a sense, the exact opposites of cardinal functions; they may be called "anti-cardinal functions."
} 
As illustrations of the identity for functions of one argument, we have

(1) since $\sigma_{a}=I_{a} \cdot E$,

$$
\sigma_{a}(M N)=\sum \sigma_{a}\left(\frac{M}{\delta}\right) \sigma_{a}\left(\frac{N}{\delta}\right) \delta^{a} \mu(\delta),
$$

summed for common divisors $\delta$, of $M$ and $N$;

(2) in particular,

$$
\sigma_{a}\left(N^{2}\right)=\sum\left\{\sigma_{a}\left(\frac{N}{\delta}\right)\right\}^{2} \delta^{a} \mu(\delta), \text { summed for divisors } \delta \text {, of } N \text {. }
$$

Generally, if $f=L_{1} \cdot L_{2}$, and $L_{12}=L_{1} \times L_{2}$, then when $M$ is a multiple of $N$,

$$
\begin{aligned}
f(M N) & =\sum f\left(\frac{M}{\delta}\right) f\left(\frac{N}{\delta}\right) L_{12}^{-1}(\delta), \\
f(M) f(N) & =\sum f\left(\frac{M N}{\delta^{2}}\right) L_{12}(\delta),
\end{aligned}
$$

the summation extending to divisors $\delta$, of $N$, in each case.

In particular, if $M=N^{k}$,

$$
\begin{aligned}
f\left(N^{k+1}\right) & =\sum f\left(\frac{N^{k}}{\delta}\right) f\left(\frac{N}{\delta}\right) L_{12}^{-1}(\delta) \\
f\left(N^{k}\right) f(N) & =\sum f\left(\frac{N^{k+1}}{\delta^{2}}\right) L_{12}(\delta)
\end{aligned}
$$

A different form may also be given to the Busche-Ramanujan identity for $f(M)=\left(L_{1} \cdot L_{2}\right)(M)$. Namely, we have

$$
\begin{aligned}
f(M N) & =f(M) f(N) \cdot \operatorname{conj} \operatorname{crd} f^{-1}(M N) \\
& =f f \cdot E^{-1} \cdot E \cdot \operatorname{princ}\left(L_{12}^{-1} \cdot E \cdot E^{-1}\right) \\
& =f f \cdot E^{-1} \cdot\left\{\left(L_{12}^{-1} \cdot E\right)(g)\right\},
\end{aligned}
$$

where $g$ is the g.c.d. of $M, N$.

For example,

$$
\begin{aligned}
\sigma_{a}(M N) & =I_{a} \cdot\left\{\left(I_{a}^{-1} \cdot E\right)(g)\right\} \\
& =\sum\left(\frac{M N}{\delta_{1} \delta_{2}}\right)^{a} F\left\{g\left(\delta_{1} \delta_{2}\right)\right\}\left(\delta_{1} / M, \delta_{2} / N\right),
\end{aligned}
$$

where $g\left(\delta_{1}, \delta_{2}\right)$ denotes the g.c.d. of $\delta_{1},{ }^{\top} \delta_{2}$ and $F(N)=$ the product $\Pi\left(1-p^{a}\right)$ extended over all the 
An alternative form of the same result is

$$
\begin{aligned}
f(M) f(N) & =f(M N) \cdot E^{-1} \cdot E \cdot \operatorname{princ}\left(L_{12} \cdot E \cdot E^{-1}\right) \\
& =f(M N) \cdot E^{-1} \cdot\left\{\left(L_{12} \cdot E\right)(g)\right\}
\end{aligned}
$$

or

$$
(f \cdot E)(M)(f \cdot E)(N)=\sum f\left(\frac{M N}{\delta_{1} \delta_{2}}\right)\left(L_{12} \cdot E\right)\left(g\left(\delta_{1}, \delta_{2}\right)\right),
$$

which is valid, when $f$ is any integral quadratic function.

5. The second and third cardinal functions. We shall next investigate the form of the functions, whose second, or third, cardinal functions become principal functions.

The second cardinal function $C_{2}\left(\left|M_{i}, N_{i}\right|\right)$ of $f\left(M_{1}, M_{2}, \cdots, M_{r}\right)$ is given by

$$
f\left(M_{1} N_{1}, M_{2} N_{2}, \cdots, M_{r} N_{r}\right)=f f \times\left(C_{2} \cdot E\right) .
$$

Writing

$$
f_{(p)}\left(x_{1}, x_{2}, \cdots, x_{r}\right)=\sum a\left(m_{1}, m_{2}, \cdots, m_{r}\right) x_{1}^{m_{1}} x_{2}^{m_{2}} \cdots x_{r}^{m_{r}},
$$

it follows that

$$
\begin{aligned}
& \left(C_{2} \cdot E\right)_{(p)}\left(x_{1}, \cdots, x_{r}, y_{1}, \cdots, y_{r}\right) \\
& \quad=\sum \frac{a\left(m_{1}+n_{1}, m_{2}+n_{2}, \cdots, m_{r}+n_{r}\right)}{a\left(m_{1}, m_{2}, \cdots, m_{r}\right) a\left(n_{1}, n_{2}, \cdots, n_{r}\right)} x_{1}^{m_{1}} x_{2}^{m_{2}} \cdots x_{r}^{m_{r}} y_{1}^{n_{1}} \cdots y_{r}^{n_{r}} .
\end{aligned}
$$

This determines $C_{2}$. If $C_{2}$ is to be a principal function, $C_{2} \cdot E$ must be a function of the g.c.d.'s $g_{i}$ of $M_{i}, N_{i}$ (Theorem XXXI). Hence

$$
\frac{a\left(m_{1}+n_{1}, m_{2}+n_{2}, \cdots, m_{r}+n_{r}\right)}{a\left(m_{1}, m_{2}, \cdots, m_{r}\right) a\left(n_{1}, n_{2}, \cdots, n_{r}\right)}
$$

must depend only on the smaller element of each of the pairs $\left(m_{1}, n_{1}\right)$, $\left(m_{2}, n_{2}\right), \cdots$. Hence, if $m_{1} \geqq n_{1}$,

$$
\frac{a\left(m_{1}+k+n_{1}, m_{2}+n_{2}, \cdots, m_{r}+n_{r}\right)}{a\left(m_{1}+k, m_{2}, \cdots, m_{r}\right) a\left(n_{1}, n_{2}, \cdots, n_{r}\right)}
$$

is independent of $k$, and is equal to

$$
\frac{a\left(m_{1}+n_{1}, \cdots, m_{r}+n_{r}\right)}{a\left(m_{1}, \cdots, m_{r}\right) a\left(n_{1}, n_{2}, \cdots, n_{r}\right)} .
$$

It follows from this that if $m_{i}>0$,

$\boldsymbol{a}\left(m_{1}, m_{2}, \cdots, m_{r}\right)=\lambda_{i}^{m_{i}-1} a\left(m_{1}, m_{2}, \cdots, m_{i-1}, 1, m_{i+1}, \cdots, m_{r}\right)(i=1,2, \cdots, r)$. 
Also

$$
\frac{a(1,1,0, \cdots, 0)}{a(0,1,0, \cdots, 0) a(1,0, \cdots, 0)}=\frac{a(0,1,0, \cdots, 0)}{a(0,1,0, \cdots, 0) a(0,0, \cdots, 0)}=1,
$$

since in both cases the lesser of $m_{i}, n_{i}$ is the same. Hence

$$
a(1,1,0, \cdots, 0)=a(0,1,0, \cdots, 0) a(1,0, \cdots, 0) .
$$

Similarly

$$
a(1,1, \cdots, 1)=a(1,0,0, \cdots, 0) \alpha(0,1,0, \cdots, 1,0) \cdots .
$$

Thus

$a\left(m_{1}, m_{2}, \cdots, m_{i}, 0, \cdots, 0\right)=\left\{\lambda_{1}^{m_{1}-1} a(1,0, \cdots, 0)\right\}\left\{\lambda_{2}^{m_{2}-1} a(0,1,0, \cdots, 0)\right\} \cdots$,

where

$$
m_{1}, m_{2}, \cdots, m_{i}>0
$$

Writing

$$
a(1,0, \cdots, 0)=\lambda_{1}-a_{1} ; a(0,1,0, \cdots, 0)=\lambda_{2}-a_{2}, \text { etc. },
$$

it follows that

$$
f_{(p)}\left(x_{1}, x_{2}, \cdots, x_{r}\right)=\frac{\left(1-a_{1} x_{1}\right)\left(1-a_{2} x_{2}\right) \cdots\left(1-a_{r} x_{r}\right)}{\left(1-\lambda_{1} x_{1}\right)\left(1-\lambda_{2} x_{2}\right) \cdots\left(1-\lambda_{r} x_{r}\right)}
$$

Thus $f$ is a totient. Also it is easy to see that $\left(C_{2} \cdot E\right)\left(\left|M_{i}, N_{i}\right|\right.$ ) now becomes $T^{\prime}\left(g_{1}, g_{2}, \cdots, g_{r}\right)$, where $T^{\prime}$ is the level totient such that $T^{\prime} \times f=$ the linear component of $f$. Hence

THEOREM XXXVI. The totients are the only functions of $r$ arguments, whose second cardinal function is a principal function. Also, if $T\left(M_{1}, M_{2}, \cdots\right.$, $\left.M_{r}\right)$ is a totient, and $T^{\prime}$ is the level totient such that $T \times T^{\prime}$ is a linear function, $T\left(M_{1} N_{1}, M_{2} N_{2}, \cdots, M_{r} N_{r}\right)$

$$
=T\left(M_{1}, M_{2}, \cdots, M_{r}\right) T\left(N_{1}, N_{2}, \cdots, N_{r}\right) T^{\prime}\left(g_{1}, g_{2}, \cdots, g_{r}\right) .
$$

For instance, if $\phi_{k}(M)$ is the Jordan function,

$$
\frac{\phi_{k}(M N)}{\phi_{k}(M) \phi_{k}(N)}=\frac{g^{k}}{\phi_{k}(g)} \text {. }
$$

More generally, if $\phi_{k, r}=I_{k} \cdot \lambda_{r}^{-1}$ is the Jordan-Schemmel totient (VII §3),

$$
\frac{\phi_{k, r}(M N)}{\phi_{k, r}(M) \phi_{k, r}(N)}=\frac{g^{k}}{\phi_{k, r}(g)} .
$$


The third cardinal function is less interesting than the other two, and becomes a principal function only in a trivial case.

Theorem XXXVII. If the third cardinal function $C_{3}$ of $f$ is a principal function, it is the principal function $E_{0}$, and $f$ must be a linear function.

For, with our previous notation, it follows that when $C_{3}$ is a principal function, we must have,

$a\left(m_{1}+n_{1}, m_{2}+n_{2}, \cdots, m_{r}+n_{r}\right)-a\left(m_{1}, m_{2}, \cdots, m_{r}\right) a\left(n_{1}, n_{2}, \cdots, n_{r}\right)=0$, whenever any $m_{i} \neq n_{i}(i=1,2, \cdots, r)$. Hence writing

$$
a_{1}=a(1,0, \cdots, 0) ; a_{2}=a(0,1,0, \cdots, 0) \text { etc. }
$$

we have

$$
a\left(m_{1}, 0, \cdots, 0\right)=a(2,0, \cdots, 0) a^{m_{1}-2} \quad\left(m_{1} \geqq 2\right),
$$

and similar equations. Also, if $m_{1} \neq 2, n_{1} \neq 2, m_{1} \neq n_{1}$,

$$
\begin{aligned}
\{a(2,0, \cdots, 0)\}^{2} a_{1}^{m_{1}+n_{1}-4} & =a\left(m_{1}, 0, \cdots, 0\right) a\left(n_{1}, 0, \cdots, 0\right) \\
& =a\left(m_{1}+n_{1}, 0, \cdots, 0\right) \\
& =a(2,0, \cdots, 0) a_{1}^{m_{1}+n_{1}-2} .
\end{aligned}
$$

Hence $a(2,0, \cdots, 0)=a_{1}^{2}$, so that $a\left(m_{1}, 0, \cdots, 0\right)=a_{1}^{m_{1}}\left(m_{1}=0,1,2, \cdots\right)$. Also, if $m_{1}, m_{2}, \cdots, m_{i} \neq 0$,

$$
\begin{aligned}
a\left(m_{1}, \cdots,\right. & \left.m_{i}, 0, \cdots, 0\right) \\
& =a\left(m_{1}, 0, \cdots, 0\right) a\left(0, m_{2}, 0, \cdots, 0\right) \cdots a\left(0, \cdots, 0, m_{i}, 0, \cdots, 0\right) \\
& =a_{1}^{m_{1}} a_{2}^{m_{2}} \cdots a_{i}^{m_{i}} .
\end{aligned}
$$

Thus $f$ is the linear function whose generating series

$$
f_{(p)}(x)=\frac{1}{\Pi\left(1-a_{i} x_{i}\right)} .
$$

6. The restricted Busche-Ramanujan identity. If $F(M, N), F^{\prime}(M, N)$ are two functions with identical derivates, then Theorems XXVIII and XXXIV state that each of $F, F^{\prime}$ is the composite of the other with a cardinal function, and also the compound of the other with a cardinal function. We can however state the relation between $F, F^{\prime}$ in the more general form, that each of $F, F^{\prime}$ can be obtained from the other by composing it first with a cardinal function, and then compounding the composite with a second cardinal function; that is,

$$
F^{\prime}=\left(F \cdot C_{1}\right) \oplus C_{2}
$$


Now, if $f(M)$ be a function of a single argument, $f(M N)$ and $f(M) f(N)$ have identical derivates. The relation between $f(M N)$ and $f(M) f(N)$ can therefore be put in the general form

$$
f(M N)=\left(f f \cdot C_{1}\right) \oplus C_{2},
$$

where $C_{1}, C_{2}$ are cardinal functions of $M, N$. It is clear that, in this relation, either of the cardinal functions $C_{1}, C_{2}$ may be arbitrarily chosen, and that the other becomes then determinate. We enquire, in regard to this equation, when it can happen that both $C_{1}$ and $C_{2}$ are principal functions.

The arithmetical significance of the assumption that $C_{1}$ and $C_{2}$ are simultaneously principal functions is clearly that $f$ satisfies a Busche-Ramanujan identity for certain restricted values of $M, N$. For it is evident that the compound of $F(M, N)$ and a principal function of $M, N$ has the same value as $F(M, N)$, whenever $M, N$ do not both contain any prime factor $p$ raised to the same power. Thus, when $C_{1}, C_{2}$ are principal functions, $f$ satisfies the Busche-Ramanujan identity

$$
f(M+N)=f f \cdot C_{1},
$$

for all such values of $M, N$ as have no common block-factor. We say in this case that $f$ admits a restricted Busche-Ramanujan identity.

To investigate when $C_{1}, C_{2}$ are both principal functions, take

$$
\begin{aligned}
f_{(p)}(x) & =1+a_{1} x+a_{2} x^{2}+\cdots, \\
\{f(M N)\}_{(p)}(x, y) & =\sum a_{m+n} x^{m} y^{n}, \\
C_{1(p)}(x, y) & =1+t_{1} x y+t_{2} x^{2} y^{2}+\cdots, \\
C_{2(p)}(x, y) & =1+k_{1} x y+k_{2} x^{2} y^{2}+\cdots .
\end{aligned}
$$

Then

$$
\begin{aligned}
\sum a_{m+n} x^{m} y^{n}= & \left(\sum a_{m} x^{m}\right)\left(\sum a_{n} y^{n}\right)\left(1+t_{1} x y+t_{2} x^{2} y^{2}+\cdots\right) \\
& +k_{1} x y+k_{2} x^{2} y^{2}+\cdots
\end{aligned}
$$

(V §1). Hence, if $m \neq n$,

$$
a_{m+n}=a_{m} a_{n}+t_{1} a_{m-1} a_{n-1}+t_{2} a_{m-2} a_{n-2}+\cdots .
$$

Hence, if $m>n+2$,

$$
\begin{aligned}
a_{m} a_{n+2}+a_{m-1} a_{n+1} t_{1}+\cdots+a_{m-n-2} t_{n+2} \\
\quad=a_{m+n+2} \\
=a_{1} a_{m+n+1}+a_{m+n} t_{1}(\text { by (A)) }
\end{aligned}
$$




$$
\begin{aligned}
= & a_{1}\left(a_{m} a_{n+1}+a_{m-1} a_{n} t_{1}+\cdots+a_{m-n-1} t_{n+1}\right) \\
& +t_{1}\left(a_{m} a_{n}+a_{m-1} a_{n-1} t_{1}+\cdots+a_{m-n} t_{n}\right) \\
= & a_{m} a_{n+2}+a_{m-1} a_{n+1} t_{1}+\cdots+a_{m-n} t_{n}\left(a_{1}^{2}+t_{1}\right)+a_{1} a_{m-n-1} t_{n+1},
\end{aligned}
$$

so that

(1)

$$
a_{m-n-2} t_{n+2}+t_{n} a_{m-n}\left(a_{2}-a_{1}^{2}-t_{1}\right)=0 \quad(m>n+2) .
$$

By varying $m$ in this equation, it follows that

$$
\frac{a_{r}}{a_{r-2}}=a \text { constant } \lambda=\frac{t_{2}}{a_{1}^{2}+t_{1}-a_{2}} \quad(r>2) .
$$

Similarly, by varying $n$, we have

$$
t_{n+2}=t_{n} t_{2}
$$

Now from (2),

therefore

$$
a_{3}=a_{1} a_{2}+a_{1} t_{1}=a_{1} \lambda,
$$

$$
\text { either } a_{1}=0 \text {, or } \lambda=a_{2}+t_{1} \text {. }
$$

Again from (2), $a_{1} a_{4}=a_{2} a_{3}$. Hence

$$
a_{2} a_{3}+t_{1}\left(a_{1} a_{2}+a_{1} t_{1}\right)=a_{1} a_{4}+a_{3} t_{1}=a_{5}=a_{2} a_{3}+a_{1} a_{2} t_{1}+a_{1} t_{2} .
$$

Hence,

$$
\text { either } a_{1}=0 \text {, or } t_{2}=t_{1}^{2} \text {. }
$$

Now, the relation $a_{m+1}-a_{m} a_{1}-a_{m-1} t_{1}=0$ holds from $m=2$ onwards, on account of (A). Hence the series $f_{(p)}(x)$ is a recurring series of the second order, its value, in finite terms, being evidently

$$
f_{(p)}(x)=\frac{1+\left(a_{2}-a_{1}{ }^{2}-t_{1}\right) x^{2}}{1-a_{1} x-t_{1} x^{2}} .
$$

There are now three possibilities to be considered.

Case 1. $a_{2}-a_{1}^{2}-\dot{t}_{1}=0$. For this case the element of $f$ to the base $p$ is that of an integral quadratic function; from (2) and (3), we easily see that $t_{n}=0$ for $n>1$. We also see that $C_{2}$ reduces to $E_{0}$, so that our restricted identity becomes the unrestricted identity, which, we know, is satisfied by every integral quadratic function.

Case 2. $a_{2}-a_{1}^{2}-t_{1} \neq 0, a_{1} \neq 0$. For this case it follows from (4), (5), that

$$
t_{2}=t_{1}^{2}, \quad \frac{t_{2}}{a_{1}^{2}+t_{1}-a_{2}}=\lambda=a_{2}+t_{1} .
$$


These imply the vanishing of $a_{2}^{2}-a_{1}^{2}\left(a_{2}+t_{1}\right)$, which is the resultant of the numerator and denominator of $f_{(p)}(x)$. Thus the element of $f$ reduces to that of a totient. Conversely, when $f$ is the totient $L_{1} \cdot L_{2}{ }^{-1}$,

$$
\begin{aligned}
f_{(p)}(x) & =\frac{1-\alpha x}{1-\beta x} ; \\
\{f(M N)\}_{(p)}(x, y) & =\frac{1-\alpha(x+y)+\alpha \beta x y}{(1-\beta x)(1-\beta y)} \\
& =\frac{(1-\alpha x)(1-\alpha y)}{(1-\beta x)(1-\beta y)(1-\alpha \beta x y)}-\frac{\alpha^{2} x y}{1-\alpha \beta x y} .
\end{aligned}
$$

Hence $f=L_{1} \cdot L_{2}^{-1}$ has the restricted identity

$$
f(M N)=\left\{f f \cdot \operatorname{princ}\left(L_{1} \times L_{2}\right)\right\} .
$$

Case 3. $a_{2}-a_{1}^{2}-t_{1} \neq 0, a_{1}=0$. Now

$$
f_{(p)}(x)=\frac{1+\left(a_{2}-t_{1}\right) x^{2}}{1-t_{1} x^{2}}
$$

so that $f$ is the convolute of a totient. Conversely, when $f$ is the second convolute of a totient,

$$
\begin{aligned}
f_{(p)}(x) & =\frac{1+\alpha x^{2}}{1-\beta x^{2}} ; \\
\{f(M N)\}_{(p)}(x, y) & =\frac{1+\alpha\left(x^{2}+y^{2}\right)-\alpha \beta x^{2} y^{2}+(\alpha+\beta) x y}{\left(1-\beta x^{2}\right)\left(1-\beta y^{2}\right)} \\
& =\frac{\left(1+\alpha x^{2}\right)\left(1+\alpha y^{2}\right)(1+\beta x y)}{\left(1-\beta x^{2}\right)\left(1-\beta y^{2}\right)\left(1+\alpha \beta x^{2} y^{2}\right)}+\frac{\alpha x y(1-\alpha x y)}{1+\alpha \beta x^{2} y^{2}} .
\end{aligned}
$$

Hence if $f$ is the second convolute of the totient $T=L_{1} \cdot L_{2}^{-1}$, it has the restricted identity

$$
f(M N)=f f \cdot \operatorname{princ} F,
$$

valid whenever $M, N$ have no common block-factor, where

$$
F(N)=\frac{L_{1}(N) L_{2}(D)}{L_{1}(D)},
$$

$D$ being the greatest number whose square divides $N$. Combining these results, we have

THEOREM XXXVIII. The only functions of $M$ which admit a restricted Busche-Ramanujan identity (namely an identity valid when $M, N$ have no common block-factor) are (1) the integral quadratic functions, (2) totients, (3) second convolutes of totients, and (4) crosses between these types. 
As illustrations of the theorem, we have the following identities, valid when $M, N$ have no common block-factor:*

$$
\phi(M N)=\sum \phi\left(\frac{M}{\delta}\right) \phi\left(\frac{N}{\delta}\right) \delta,
$$

summed for common divisors $\delta$, of $M, N$, where $\phi$ is Euler's function.

$$
\operatorname{conv} \phi(M N)=\sum \operatorname{conv} \phi\left(\frac{M}{\delta}\right) \operatorname{conv} \phi\left(\frac{N}{\delta}\right) F(\delta),
$$

where $F(N)$ is the least divisor of $N$, which is divisible by its complementary divisor.

\section{Section VII. The theory of Smith's Determinant}

1. Ordinal functions of $r$ arguments. A function $f\left(M_{1}, M_{2}, \cdots, M_{r}\right)$ of $r$ arguments may be called generally an ordinal function, if it vanishes whenever certain prescribed inequalities of the form $M_{i}>M_{j}$ hold between the arguments. Since we restrict ourselves to multiplicative functions, this property may be shown to imply a more specialized property of $f$.

THEOREM XXXIX. If a multiplicative function of $M_{1}, M_{2}, \cdots, M_{r}$ vanishes whenever $M_{i}>M_{j}$, it necessarily vanishes whenever $M_{i}$ is not a factor of $M_{j}$.

For by hypothesis, $f\left(p^{m_{1}}, p^{m_{2}}, \cdots, p^{m_{r}}\right)$ vanishes, for any prime $p$, whenever $m_{i}>m_{j}$; that is, unless $p^{m_{i}}$ is a factor of $p^{m_{i}}$. Hence $f\left(M_{1}, M_{2}, \cdots, M_{r}\right)$ vanishes if any prime $p$ occurs to a higher power in $M_{i}$ than in $M_{i}$, that is, unless $M_{i}$ is a factor of $M_{j}$.

We shall concern ourselves only with two types of ordinal functions; namely, the functions $f\left(M_{1}, M_{2}, \cdots, M_{r}\right)$, which vanish whenever a prescribed argument $M_{i}$ is greater than any of the remaining arguments, and functions $F\left(M_{1}, M_{2}, \cdots, M_{r}\right)$ which vanish whenever a prescribed argument $M_{j}$ is less than any of the other arguments. We call $f\left(M_{1}, M_{2}, \cdots, M_{r}\right)$ a minor ordinal function with the minor argument $M_{i}$, and $F\left(M_{1}, M_{2}, \cdots, M_{r}\right)$ a major ordinal function with the major argument $M_{j}$. We shall usually indicate the major or minor argument by writing it without the suffix.

2. Major ordinal functions and functions with a modulus. There exist multiplicative functions $f\left(M_{1}, M_{2}, \cdots, M_{r-1}, M\right)$, whose value is unaltered if $M_{i}$ is increased by any multiple of $M(i=1,2, \cdots, r-1)$; such functions may be said to possess the modulus $M$. We shall now show that, with each

* These two results (the first of which is due to Mr. S. S. Pillai, as already stated) have been published as questions for solution in the Journal of the Indian Mathematical Society (December, 1928, last page, Nos. (1529) and (1530)). 
function $f\left(M_{1}, M_{2}, \cdots, M_{r-1}, M\right)$ possessing the modulus $M$, we can associate a major ordinal function $F\left(M_{1}, M_{2}, \cdots, M_{r-1}, M\right)$ with the major argument $M$, in such a way that

$$
f\left(M_{1}, M_{2}, \cdots, M_{r-1}, M\right)=F\left(g_{1}, g_{2}, \cdots, g_{r-1}, M\right),
$$

where $g_{i}$ is the g.c.d. of $M_{i}, M(i=1,2, \cdots, r-1)$.

For, since $f\left(M_{1}, M_{2}, \cdots, M_{r-1}, M\right)$ has the modulus $M$, and is a multiplicative function of its arguments, it follows that

$$
f\left(M_{1}, M_{2}, \cdots, M_{r-1}, 1\right)=f(1,1, \cdots, 1) .
$$

Hence $D_{M}(f)=E$, so that by Theorem XXVI, $f$ is a multiplicative function of its modulus $M$ alone. Further, let $N$ be any number prime to the modulus $M$. Then by a well known theorem, $M_{1}, M_{1}+M, M_{1}+2 M, \cdots$, $M_{1}+(N-1) M$ is a complete residue system $\bmod N$. Hence there exist integers $\lambda_{1}, \lambda_{2}, \cdots, \lambda_{r-1}$, such that $M_{i}+\lambda_{i} M$ is prime to $N(i=1,2, \cdots, r-1)$. Hence

$$
\begin{aligned}
f\left(M_{1}, M_{2},\right. & \left.\cdots, M_{r-1}, M\right) \\
& =f(1,1, \cdots, 1) f\left(M_{1}, M_{2}, \cdots, M_{r-1}, M\right) \\
& =f(N, 1, \cdots, 1) f\left(M_{1}+\lambda_{1} M, M_{2}+\lambda_{2} M, \cdots, M_{r-1}+\lambda_{r-1} M, M\right) \\
& =f\left(M_{1} N+\lambda_{1} M N, M_{2}+\lambda_{2} M, \cdots, M_{r-1}+\lambda_{r-1} M, M\right)
\end{aligned}
$$

(since $f$ is a multiplicative function of its $r$ arguments)

$$
=f\left(M_{1} N, M_{2}, \cdots, M_{r-1}, M\right)
$$

(since $f$ has the modulus $M$ ).

Hence the $r-1$ arguments $M_{1}, M_{2}, \cdots, M_{r-1}$ of a function $f\left(M_{1}\right.$, $\left.M_{2}, \cdots, M_{r-1}, M\right)$ with the modulus $M$ can not only be increased by multiples of $M$, but can also be multiplied by any number prime to $M$, without affecting the value of the function. Hence $f$ depends on $M_{1}, M_{2}, \cdots, M_{r-1}$ only through their g.c.d.'s with $M$.

Now, if $f\left(M_{1}, M_{2}, \cdots, M_{r-1}, M\right)$ be any multiplicative function, we can define a function $F\left(M_{1}, M_{2}, \cdots, M_{r-1}, M\right)$ by

$$
F\left(M_{1}, M_{2}, \cdots, M_{r-1}, M\right)=f\left(M_{1}, M_{2}, \cdots, M_{r-1}, M\right),
$$

when each $M_{i}$ is a factor of $M$,

$$
=0 \text {, in other cases. }
$$

The multiplicative character of $F$ follows from that of $f$. Thus an arbitrary function $f\left(M_{1}, M_{2}, \cdots, M_{r-1}, M\right)$ defines a major ordinal function $F\left(M_{1}, \cdots\right.$, 
$\left.M_{r-1}, M\right)$; but it is obvious that $F$ does not determine $f$ uniquely. If, however, $f\left(M_{1}, M_{2}, \cdots, M_{r-1}, M\right)$ possesses the modulus $M$, then from what we have proved, $f$ both determines and is determined uniquely by $F$.

As an illustration, we may mention von Sterneck's function $f(N, M)$ which represents the excess of the number of partitions of $N$ into an even number of parts mod $M$, over the number of those into an odd number. This function can be shown to be multiplicative, and possesses, by definition, the modulus $M$. Hence it must be a multiplicative function of the modulus alone, and can be expressed as a function of $M$ and its g.c.d. with $N .^{*}$

3. Minor ordinal functions. If $f\left(M, M_{1}, \cdots, M_{r-1}\right)$ is a minor ordinal function with the minor argument $M$, we shall call $\psi(M)=f(M, M, \cdots, M)$ the kernel of $f$.

We can associate with every minor ordinal function $f\left(M, M_{1}, \cdots, M_{r-1}\right)$ a general multiplicative function $F\left(M, N_{1}, N_{2}, \cdots, N_{r-1}\right)$, of $r$ arguments, defined by

$$
F\left(M, N_{1}, N_{2}, \cdots, N_{r-1}\right)=f\left(M, M N_{1}, M N_{2}, \cdots, M N_{r-1}\right) .
$$

Conversely, from any multiplicative function $F\left(M, N_{1}, \cdots, N_{r-1}\right)$, we can define a minor ordinal function $f\left(M, M_{1}, \cdots, M_{r-1}\right)$ by

$f\left(M, M_{1}, \cdots, M_{r-1}\right)=F\left(M, \frac{M_{1}}{M}, \frac{M_{2}}{M}, \ldots, \frac{M_{r-1}}{M}\right)$, when $M$ divides each $M_{i}$,

$$
=0 \text {, otherwise. }
$$

Thus the minor ordinal functions are in reversible one-to-one correspondence with all multiplicative functions. We observe, in particular, that the derivate of the function $F\left(M, N_{1}, N_{2}, \cdots, N_{r-1}\right)$ with respect to $N_{1}, N_{2}, \cdots, N_{r-1}$ is the kernel of the corresponding ordinal function $f\left(M, M_{1}, \cdots, M_{r-1}\right)$.

The case in which the associated function $F\left(M, N_{1}, \cdots, N_{r-1}\right)$ is of the form $\psi(M) \psi\left(N_{1}, N_{2}, \cdots, N_{r-1}\right)$ is of special importance. For this case we have

THEOREM XL. When the associated function of the ordinal function $f\left(M, M_{1}, \cdots, M_{r-1}\right)$, with the minor argument $M$, is of the form $\psi(M) \psi\left(N_{1}\right.$, $\left.N_{2}, \cdots, N_{r-1}\right)$, then

(1) $\psi$ is the kernel of $f$,

(2) the ordinal function $f$ is the composite of princ $\psi$ and a function of $M_{1}, M_{2}, \cdots, M_{r-1}$ only.

Conversely, the composite of a principal function $P$ of $r$ arguments, and

* See Bachmann, Niedere Zahlen-Theorie, part 2, p. $230 \mathrm{ff}$. 
any function of $r-1$ of the arguments, is a minor ordinal function, whose kernel is the equivalent function of $P$.

The first part of the theorem follows from the fact that the kernel is the derivate of the associated function. To prove the second part, let the associated function have the generating series

$$
F_{(p)}\left(x, x_{1}, \cdots, x_{r-1}\right)=\sum a_{m} b_{m_{1} m_{2}} \cdots m_{r-1} x^{m} x_{1}^{m_{1}} \cdots x_{r-1}^{m_{r-1}} .
$$

Then, the generating series to the same base of the minor ordinal function is evidently

$$
\begin{aligned}
\sum a_{m} b_{m_{1} m_{2}} \cdots m_{r-1} & x^{m} x_{1}^{m+m_{1}} x_{2}^{m_{+m}} \cdots x_{r-1}^{m+m_{r-1}} \\
& =\left\{\sum a_{m}\left(x x_{1} \cdots x_{r-1}\right)^{m}\right\}\left\{\sum b_{m_{1} m_{2} \cdots m_{r-1}} x_{1}^{m_{1}} x_{2}^{m_{2}} \cdots x_{r-1}^{m_{r-1}}\right\},
\end{aligned}
$$

which proves the theorem.

Definition. The composite of a minor ordinal function with any function of its minor argument $M$, will be called a Smith function in respect to $M$. Also, the kernel of the ordinal function will be termed the kernel of the coresponding Smith function.

It follows from this definition, that, if $S\left(M, M_{1}, \cdots, M_{r-1}\right)$ is a Smith function in respect to $M$, there exists a function $\psi(M)$, such that $S \cdot \psi$ is a minor ordinal function with the minor argument $M$.

4. Smith's determinant. Let $S\left(M, M_{1}, \cdots, M_{r-1}\right)$ be a Smith's function in respect to $M$. The values taken by $S$ when its $r$ arguments range from 1 to $m$ may be taken as the elements of an $r$-dimensional matrix of the $m$ th order, $\left|S\left(M, M_{1}, \cdots, M_{r-1}\right)\right|$, the $r$ arguments of $S$ serving as the indices of the matrix. This matrix can be evaluated as a determinant, if we assign a signant or a non-signant character to each index.* We shall call the de-

* The modern theory of the $r$-dimensional determinant

$$
\left|a\left(m_{1}, m_{2}, \cdots, m_{r}\right)\right| \quad\left(m_{1}, m_{2}, \cdots, m_{r}=1,2, \cdots, n\right)
$$

assigns either a signant or a non-signant character to each index $m$. The value of the determinant is

$$
\frac{1}{n !} \sum \pm a\left(m_{11}, m_{21}, \cdots, m_{r 1}\right) a\left(m_{12}, m_{22}, \cdots, m_{r 2}\right) \cdots a\left(m_{1 n}, m_{2 n}, \cdots, m_{r n}\right),
$$

summed for all permutations $\left(m_{i 1} m_{i 2} \cdots m_{i n}\right)$ of $1,2, \cdots, n(i=1,2, \cdots, r)$, so that there are $(n !) r$ terms in the summation. If $\epsilon_{i}$ represents +1 or -1 according as $\left(m_{i 1} m_{i 2} \cdots m_{i n}\right)$ is an even or odd permutation of $1,2, \cdots, n$, the sign of the general term is defined to be $\prod_{i}$, where the product extends over those values of $i$ for which $m_{i}$ is a signant index.

The theory is due to Rice ( $P$-way determinants with an application to transvectants, American Journal of Mathematics, vol. 40 (1918)) and was also discovered independently by Lecat and the present writer (On mixed determinants, Proceedings of the Royal Society of Edinburgh, 1925). For further information reference may be made to the works of Lecat, e.g. Coup d'Oeil sur la Theorie des Déterminants Supérieurs, Bruxelles, 1927, and also to a recent article of Lecat, Le déterminant superieur, qu'est il exactement? Les conceptions de Cayley, Gasparis, Rice et autres, Revue Générale des Sciences, 1929. 
terminant $\left|S\left(M, M_{1}, \cdots, M_{r-1}\right)\right|$ a Smith determinant, if the signant character has been assigned to the index $M$.

ThEOREM XLI. The value of the Smith determinant $\left|S\left(M, M_{1}, \cdots, M_{r-1}\right)\right|$ $=\Delta_{m}$, of order $m$, is zero if the number of signant indices is odd, and is $F(1) F(2) \cdots F(m)$ otherwise, $F$ being the kernel of $S$.

The first part follows from the fact that a determinant vanishes identically, unless it has an even number of signant indices.

The elements of the Smith determinant $\Delta_{m}$, in which the index $M$ has the value $k$, constitute an $(r-1)$-dimensional matrix of the $m$ th order, which we may call the $k$ th section of $\Delta_{m}$. Since $M$ is a signant index in $\Delta_{m}$, it results that we may add to the elements of a section, any the same multiples of the corresponding elements of any other section. Also, since $S$ is a Smith function, there exists a function $\psi(M)$, such that $S \cdot \psi$ is an ordinal function with the minor argument $M$. Therefore if we add to the elements of the $m$ th section $\psi(m / \delta)$ times the $\delta$ th section (where $\delta$ represents successively each divisor of $m$ other than $m$ itself), and denote the new elements of the $m$ th section by $S^{\prime}\left(m, M_{1}, \cdots, M_{r-1}\right)$, we have

$$
\begin{aligned}
S^{\prime}\left(m, M_{1}, \cdots\right. & \left., M_{r-1}\right) \\
= & (S \cdot \psi)\left(m, M_{1}, \cdots, M_{r-1}\right) \\
= & 0, \text { if any } M_{i} \neq m, \\
= & (S \cdot \psi)(m, m, \cdots, m) \\
= & F(m), \text { if } M_{1}=M_{2}=\cdots=M_{r-1}=m .
\end{aligned}
$$

Now, expanding the determinant in terms of the elements of the $m$ th section, it follows immediately that

$$
\begin{aligned}
\Delta_{m} & =F(m) \Delta_{m-1} \\
& =k F(m) F(m-1) \cdots F(2) F(1),
\end{aligned}
$$

where $k$ is easily seen to be zero or 1, according as the total number of signant indices is even or odd.

It will be noticed that the result does not depend on whether the signant or non-signant character is assigned to the remaining indices.

The following are applications of the theorem:

(1) Any function $F(g)$ of the g.c.d. of $M_{1}, M_{2}, \cdots, M_{r}$ is a Smith function in respect to any of the arguments, with the kernel $F \cdot E^{-1}$. For, 


$$
F(g)=E \cdot \operatorname{princ}\left(F \cdot E^{-1}\right),
$$

therefore

$$
E^{-1}\left(M_{i}\right) \cdot F(g)=E\left(M_{1}, \cdots, M_{i-1}, M_{i+1}, \cdots, M_{r}\right) \cdot \operatorname{princ}\left(F \cdot E^{-1}\right)
$$

$=$ an ordinal function, with the minor argument $M_{i}$, and the kernel $F E^{-1}$. Hence the corresponding Smith determinant of order $m$ and any number of dimensions has the value

$$
\left(F \cdot E^{-1}\right)(m) \times\left(F \cdot E^{-1}\right)(m-1) \times \cdots,
$$

if the number of signant indices is even.

(2) Any linear function $L(l)$ of the l.c.m. $l$ of two arguments $M, N$ is a Smith function with respect to $M$ or $N$. For

$$
\begin{aligned}
L(l) & =E \cdot \operatorname{semiprinc}\left(L \cdot E^{-1}\right) \\
& =E \cdot\left\{\left(L \cdot E^{-1}\right)(M)\left(L \cdot E^{-1}\right) N\right\} \cdot \operatorname{princ}\left\{\left(E \cdot L^{-1}\right) \times L\right\}(\text { Example 12) } \\
& =L(M) L(N) \cdot \operatorname{princ}\left\{\left(E \cdot L^{-1}\right) \times L\right\} .
\end{aligned}
$$

Hence

$$
\begin{aligned}
L^{-1}(M) \cdot L(l)= & L(N) \cdot \operatorname{princ}\left\{\left(E \cdot L^{-1}\right) \times L\right\} \\
= & \text { an ordinal function with the minor argument } M, \text { and kernel } \\
& \left(E \cdot L^{-1}\right) \times L .
\end{aligned}
$$

Hence the Smith determinant of order $m$ and two dimensions formed with the elements $L(g)$, has the value

$$
\Pi L(j)\left(E \cdot L^{-1}\right)(j) \quad(j=1,2, \cdots, m) .
$$

In particular, if $L=I$, the value is

$$
\Pi \phi(j) \pi(j) \quad(j=1,2, \cdots, m) \quad(\text { Cesàro })^{*}
$$

where $\Pi(j)$ is the product of the negatives of the prime factors of $j$.

(3) Von Sterneck's function $f(N, M)$, which is equal to the excess of the number of partitions of $N$ into an even number of distinct parts $\bmod M$, over the number of those into an odd number (zero not being admitted as a part), is a multiplicative function with the modulus $M$, and may be shown to be equal to $E^{-1}(M) E(N)$. princ $I$. $\dagger$ Hence it is a Smith function with the kernel $I$, the value of the corresponding Smith determinant being therefore $m !$.

* Dickson, p. 128, 61 .

† Cf. Bachmann, loc. cit. 
(4) If $f(M)=\left(L_{1} \cdot L_{2}\right)(M)$ is an integral quadratic function, $f(M N)$ is a Smith function with the kernel $\left(L_{1} \times L_{2}\right)^{-1}$; for, from the Busche-Ramanujan identity,

$$
f(M N)=f(M) f(N) \cdot \operatorname{princ}\left(L_{1} \times L_{2}\right)^{-1} .
$$

Since the kernel is the inverse of a linear function, it vanishes for all numbers with a squared factor, and so the corresponding Smith determinant of order $m$ vanishes unless $m<4$.

Madras University, Madras, India 